\title{
MATRIX FACTORIZATIONS AND SEMI-ORTHOGONAL DECOMPOSITIONS FOR BLOWING-UPS
}

\author{
VALERY A. LUNTS AND OLAF M. SCHNÜRER
}

\begin{abstract}
We study categories of matrix factorizations. These categories are defined for any regular function on a suitable regular scheme. Our paper has two parts. In the first part we develop the foundations; for example we discuss derived direct and inverse image functors and $\mathrm{dg}$ enhancements. In the second part we prove that the category of matrix factorizations on the blowing-up of a suitable regular scheme $X$ along a regular closed subscheme $Y$ has a semi-orthogonal decomposition into admissible subcategories in terms of matrix factorizations on $Y$ and $X$. This is the analog of a well-known theorem for bounded derived categories of coherent sheaves, and is an essential step in our forthcoming article [LSb] which defines a Landau-Ginzburg motivic measure using categories of matrix factorizations. Finally we explain some applications.
\end{abstract}

\section{Contents}

1. Introduction 1

2. Categories of curved dg sheaves 4

3. Semi-orthogonal decompositions for matrix factorizations arising from projective space bundles and blowing-ups 34

Appendix A. Admissible subcategories and semi-orthogonal decompositions $\quad 52$

Appendix B. Embeddings of Verdier quotients 56

$\begin{array}{ll}\text { References } & 59\end{array}$

\section{INTRODUCTION}

Let $X$ be a separated regular Noetherian scheme of finite Krull dimension over a field $k$, for example a regular quasi-projective scheme over $k$. Let $W \in \Gamma\left(X, \mathcal{O}_{X}\right)$ be a regular function on $X$. A matrix factorization $E$ of $W$ is a diagram

$$
E=\left(E_{1} \underset{e_{0}}{\stackrel{e_{1}}{\rightleftarrows}} E_{0}\right)
$$

of locally free sheaves of finite type (= vector bundles) on $X$ such that $e_{0} e_{1}=W \mathrm{id}_{E_{1}}$ and $e_{1} e_{0}=W \operatorname{id}_{E_{0}}$. These diagrams are the objects of a differential $\mathbb{Z}_{2}$-graded category. Its homotopy category is a triangulated category, and the category $\mathbf{M F}(X, W)$ of matrix factorizations of $W$ is defined as a certain Verdier quotient of this triangulated category, see [Orl12]. 
Let $\pi: \widetilde{X} \rightarrow X$ be the blowing-up of $X$ along a regular equi-codimensional closed subscheme $Y$. Consider the pullback diagram

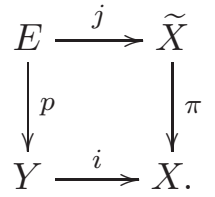

The usual construction of the blowing-up endows $\widetilde{X}$ with a line bundle $\mathcal{O}_{\widetilde{X}}(1)$. We denote its restriction to $E$ by $\mathcal{O}_{E}(1)$. We denote the pullback functions of $W$ to $Y, \widetilde{X}$ and $E$ by the same symbol. Then $\pi$ and $p$ induce (left derived) inverse image functors $\pi^{*}: \mathbf{M F}(X, W) \rightarrow$ $\mathbf{M F}(\widetilde{X}, W)$ and $p^{*}: \mathbf{M F}(Y, W) \rightarrow \mathbf{M F}(E, W)$. Similarly, $j$ gives rise to a (right derived) direct image functor $j_{*}: \mathbf{M F}(E, W) \rightarrow \mathbf{M F}(\tilde{X}, W)$ (strictly speaking this functor does not land in $\operatorname{MF}(\widetilde{X}, W)$ but in an equivalent bigger category). Now we can state our main theorem. It is the analog of a well-known result for bounded derived categories of coherent sheaves.

Theorem 1.1 (see Theorem 3.5). Assume that the codimension $r$ of $Y$ in $X$ is $\geq 2$, and let $l \in \mathbb{Z}$. Then the functors $\pi^{*}: \mathbf{M F}(X, W) \rightarrow \mathbf{M F}(\widetilde{X}, W)$ and

$$
j_{*}\left(\mathcal{O}_{E}(l) \otimes p^{*}(-)\right): \mathbf{M F}(Y, W) \rightarrow \mathbf{M F}(\widetilde{X}, W)
$$

are full and faithful. Their essential images $\pi^{*} \mathbf{M F}(X, W)$ and $\mathbf{M F}(Y, W)_{l}$ in $\mathbf{M F}(\widetilde{X}, W)$ are admissible subcategories, and we have a semi-orthogonal decomposition

$$
\mathbf{M F}(\tilde{X}, W)=\left\langle\mathbf{M F}(Y, W)_{-r+1}, \ldots, \mathbf{M F}(Y, W)_{-1}, \pi^{*} \mathbf{M F}(X, W)\right\rangle .
$$

This result is proved in the second part (section 3) of this article. As a predecessor we prove Theorem 3.2 which provides semi-orthogonal decompositions for projective space bundles. We also discuss some applications.

In the first part (section 2) we discuss general results on categories of matrix factorizations. Certainly categories of global matrix factorizations have been around for a while [LP11, Orl12] but there is no systematic treatment of the general theory, with the exception of [Pos11a, Pos11b] which contains many of our results (usually in a more general context). Here is an outline of the main results. First we define triangulated categories $\operatorname{DCoh}(X, W)$ and $\operatorname{DQcoh}(X, W)$ in essentially the same way as $\mathbf{M F}(X, W)$ by using coherent (resp. quasi-coherent) sheaves instead of vector bundles. There are natural functors

$$
\operatorname{MF}(X, W) \rightarrow \operatorname{DCoh}(X, W) \rightarrow \operatorname{DQcoh}(X, W) .
$$

We show that the first functor is an equivalence and the second one is full and faithful (see Theorem 2.9).

Assume that $Y$ is another separated regular Noetherian scheme of finite Krull dimension over $k$. Let $\pi: Y \rightarrow X$ be a morphism of schemes over $k$. The usual direct and inverse image functors $\pi_{*}$ and $\pi^{*}$ between categories of quasi-coherent sheaves give rise to functors $\mathbf{R} \pi_{*}: \operatorname{DQ} \operatorname{coh}(Y, W) \rightarrow \mathrm{DQ} \operatorname{coh}(X, W)$ and $\mathbf{L} \pi^{*}: \operatorname{DQcoh}(X, W) \rightarrow \mathrm{DQ} \operatorname{coh}(Y, W)$. This is deduced from the general theory of derived functors. Moreover, there is an adjunction $\left(\mathbf{L} \pi^{*}, \mathbf{R} \pi_{*}\right)$ (see Theorem 2.35). Similarly, we define functors $\mathbf{R} \mathcal{H o m}(-,-)$ and $\left(-\otimes^{\mathbf{L}}-\right)$. 
We then describe several (differential $\mathbb{Z}_{2}$-graded) enhancements of $\mathbf{M F}(X, W)$ (and DQcoh $(X, W)$ ) and show that they are equivalent (see section 2.6). They are constructed using injective quasi-coherent sheaves, Drinfeld dg quotient categories, and Čech resolutions, respectively. Finally we show that the subcategory of compact objects in $\operatorname{DQcoh}(X, W)$ is the Karoubi envelope of $\mathbf{M F}(X, W)$, and that $\mathbf{M F}(X, W)$ has a classical generator (see section 2.7).

In two appendices we collect some results on admissible subcategories and semi-orthogonal decompositions (appendix A) and on embeddings of Verdier quotients (appendix B).

This article is part of our project to construct motivic measures using categories of matrix factorizations. We sketch our main results. They will appear in forthcoming articles.

We now assume that $k$ is algebraically closed and of characteristic zero. Denote by $K_{0}\left(\operatorname{Var}_{\mathbb{A}^{1}}\right)$ the motivic Grothendieck group of varieties over $\mathbb{A}^{1}:=\mathbb{A}_{k}^{1}$. Given $W: X \rightarrow$ $\mathbb{A}^{1}$ and $V: Y \rightarrow \mathbb{A}^{1}$ we define $W * V: X \times Y \rightarrow \mathbb{A}^{1}$ by $(W * V)(x, y)=W(x)+V(y)$. This operation turns $K_{0}\left(\operatorname{Var}_{\mathbb{A}^{1}}\right)$ into a commutative ring. By a Landau-Ginzburg motivic measure we mean a morphism of rings from $K_{0}\left(\operatorname{Var}_{\mathbb{A}^{1}}\right)$ to some other ring.

Given a smooth variety $X$ and $W: X \rightarrow \mathbb{A}^{1}$ we define the category of singularities of $W$ as

$$
\mathbf{M F}(W):=\prod_{a \in k} \mathbf{M F}(X, W-a) .
$$

Only finitely many factors of this product are non-zero, and $\mathbf{M F}(W)$ vanishes if and only if $W$ is a smooth morphism. Let $\mathbf{M F}(W)^{\mathrm{dg}, \boldsymbol{h}}$ be a suitable enhancement of the Karoubi envelope of $\mathbf{M F}(W)$. If $W$ is a proper morphism, $\mathbf{M F}(W)^{\mathrm{dg}, \mathfrak{t}}$ is a saturated dg (= differential $\mathbb{Z}_{2}$-graded) category.

We denote by $K_{0}\left(\mathrm{sat}_{k}^{\mathbb{Z}_{2}}\right)$ the free abelian group generated by the quasi-equivalence classes of saturated $\operatorname{dg}$ (= differential $\mathbb{Z}_{2}$-graded) categories with relations coming from semiorthogonal decompositions into admissible subcategories on the level of homotopy categories. The tensor product of dg categories induces a ring structure on $K_{0}\left(\mathrm{sat}_{k}^{\mathbb{Z}_{2}}\right)$. One may think of $K_{0}\left(\mathrm{sat}_{k}^{\mathbb{Z}_{2}}\right)$ as a Grothendieck ring of suitable pretriangulated dg categories. Here is the main result of the forthcoming article [LSb].

Theorem 1.2. There is a unique morphism

$$
\mu: K_{0}\left(\operatorname{Var}_{\mathbb{A}^{1}}\right) \rightarrow K_{0}\left(\mathrm{sat}_{k}^{\mathbb{Z}_{2}}\right)
$$

of rings (= a Landau-Ginzburg motivic measure) that maps $[X, W]$ to the class of $\mathbf{M F}(W)^{\mathrm{dg}, \boldsymbol{h}}$ whenever $X$ is a smooth variety and $W: X \rightarrow \mathbb{A}^{1}$ is a proper morphism.

In particular, $\mu$ is a morphism of abelian groups and maps $[X, W]$ to the class of $\mathbf{M F}(W)^{\mathrm{dg}, \boldsymbol{\natural}}$ whenever $X$ is a smooth (connected) variety and $W: X \rightarrow \mathbb{A}^{1}$ is a projective morphism. These two properties determine $\mu$ uniquely.

Since $K_{0}\left(\operatorname{Var}_{\mathbb{A}^{1}}\right)$ has a presentation whose relations come from suitable blowing-ups (see [Bit04, Thm. 5.1]), Theorem 1.1 and its predecessor Theorem 3.2 essentially imply that there is a unique morphism $\mu: K_{0}\left(\operatorname{Var}_{\mathbb{A}^{1}}\right) \rightarrow K_{0}\left(\operatorname{sat}_{k}^{\mathbb{Z}_{2}}\right)$ of abelian groups sending $[X, W]$ to the class of $\mathbf{M F}(W)^{\mathrm{dg}, t}$ if $X$ is a smooth variety and $W$ is a proper morphism. Here we implicitly use the fact mentioned above that $\mathbf{M F}(W)^{\mathrm{dg}, 4}$ is a saturated dg category for proper $W$. This fact and multiplicativity of $\mu$ is established in [LSb]. We also give a careful definition of $K_{0}\left(\mathrm{sat}_{k}^{\mathbb{Z}_{2}}\right)$ there. 
Theorem 1.2 above was motivated by and is a relative version of a result by A. Bondal, M. Larsen and the first author (see [BLL04, 8.2]): they construct a morphism of rings

$$
K_{0}\left(\operatorname{Var}_{k}\right) \rightarrow K_{0}\left(\operatorname{sat}_{k}^{\mathbb{Z}}\right)
$$

(= a motivic measure) that maps the class of a smooth projective variety $X$ over $k$ to the class of the standard enhancement of $D^{b}(\operatorname{Coh}(X))$ by bounded below complexes of injective sheaves with bounded coherent cohomologies; here $K_{0}\left(\operatorname{Var}_{k}\right)$ is the Grothendieck group of varieties over $k$, and $K_{0}\left(\right.$ sat $\left._{k}^{\mathbb{Z}}\right)$ is defined similarly as $K_{0}\left(\right.$ sat $\left._{k}^{\mathbb{Z}_{2}}\right)$ starting from saturated differential $\mathbb{Z}$-graded categories.

In the article $[\mathrm{Sch}]$ we show that the above two motivic measures are connected by a commutative diagram

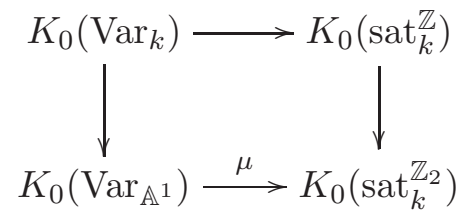

of ring morphisms where the vertical morphism on the left maps $[X]$ to $[X, 0]$ and the vertical morphism on the right is induced by folding a differential $\mathbb{Z}$-graded category into a differential $\mathbb{Z}_{2}$-graded category (and taking its triangulated envelope). The upper (resp. lower) horizontal arrow maps $\mathbb{L}_{k}:=\left[\mathbb{A}^{1}\right]$ (resp. $\mathbb{L}_{\mathbb{A}^{1}}:=\left[\mathbb{A}^{1}, 0\right]$ ) to 1 .

In the article $[\mathrm{LSa}]$ we prove that the motivic vanishing fiber map

$$
\phi: K_{0}\left(\operatorname{Var}_{\mathbb{A}^{1}}\right) \rightarrow \mathcal{M}_{k}^{\hat{\mu}}
$$

to the equivariant Grothendieck ring $\mathcal{M}_{k}^{\hat{\mu}}$ is also a Landau-Ginzburg motivic measure (here $\hat{\mu}$ is the projective limit of the group schemes $\mu_{n}$ of $n$-th roots of unity). We show that it is related to the above measure (1.1) via Euler characteristics with compact support on one hand and Euler characteristics of periodic cyclic homology on the other hand.

Acknowledgments. We thank Maxim Kontsevich for sharing his insights with us and for many useful discussions. We thank Alexander Kuznetsov for explanations concerning Theorem 3.4. Furthermore we have benefited from discussions and correspondence with Dmitri Orlov, Vladimir Drinfeld, Daniel Huybrechts, Tobias Dyckerhoff, Bertrand Toën and János Kollár.

The second author was supported by a postdoctoral fellowship of the German Academic Exchange Service (DAAD) when finishing this article. Before that he was partially supported by the Collaborative Research Center SFB Transregio 45 and the priority program SPP 1388 of the German Science foundation (DFG). He thanks these institutions.

\section{Categories of Curved DG Sheaves}

As described in the introduction we discuss foundational results on categories of matrix factorizations. Our main references for this section were [Pos11a, Pos11b, Orl12]. Some of the ideas are also contained in [LP11].

Let $k$ be a fixed field. All schemes considered are schemes over $k$. We say that a scheme $X$ satisfies condition ( $\mathrm{srNfKd}$ ) if 
$(\operatorname{srNfKd}) X$ is a separated regular Noetherian scheme of finite Krull dimension.

For example, any regular quasi-projective scheme satisfies condition (srNfKd). Note that any coherent $\mathcal{O}_{X}$-module on an (srNfKd)-scheme $X$ is a quotient of a locally free $\mathcal{O}_{X}$-module of finite type (by theorems of Kleiman [Har77, Ex. III.6.8] and Auslander and Buchsbaum [Mat89, Thm. 20.3]); in particular, such a scheme satisfies condition (ELF) in [Orl12].

Fix a scheme $X$ satisfying condition $(\operatorname{srNfKd})$. Let $W \in \Gamma\left(X, \mathcal{O}_{X}\right)$ be a global regular function which we consider as a morphism $W: X \rightarrow \mathbb{A}^{1}:=\mathbb{A}_{k}^{1}=\operatorname{Spec} k[T]$. We do not assume that the morphism $W$ is flat, for example $W$ may be the zero function.

In this section graded means $\mathbb{Z}_{2}$-graded (where $\mathbb{Z}_{2}=\mathbb{Z} / 2 \mathbb{Z}$ ) if not explicitly stated otherwise, and differential graded is often abbreviated by $\mathrm{dg}$. We use lower indices when referring to the graded components of a $\mathbb{Z}_{2}$-graded object.

The usual notions and results for differential $\mathbb{Z}$-graded categories (quasi-equivalence, pretriangulated dg category, (Drinfeld) dg quotient, etc.) have obvious counterparts in the world of differential $\mathbb{Z}_{2}$-graded categories.

2.1. Definition of various categories. By a sheaf on $X$ we mean an $\mathcal{O}_{X}$-module. We denote by $\operatorname{Sh}(X)$ the category of all sheaves on $X$, and by $\mathrm{Qcoh}(X)$ and $\operatorname{Coh}(X)$ the full subcategories of quasi-coherent and coherent sheaves, respectively. By $\operatorname{Inj} \operatorname{Sh}(X)$ (resp. $\operatorname{InjQcoh}(X))$ we denote the full subcategory of injective objects in $\operatorname{Sh}(X)(\operatorname{resp} . \mathrm{Qcoh}(X))$. We write $\operatorname{Locfree}(X)$ (resp. FlatQcoh $(X)$ ) for the full subcategory of Qcoh $(X)$ consisting of locally free sheaves (of possibly infinite rank) (resp. of quasi-coherent sheaves that are flat over $\left.\mathcal{O}_{X}\right)$.

We recall some results from [Har66, II.§7] and deduce some well-known consequences.

Theorem 2.1 ([Har66, II. $\S 7])$. (Here X can be any locally Noetherian scheme.)

(a) Every object of $\mathrm{Qcoh}(X)$ can be embedded in an object of $\operatorname{Inj} \operatorname{Sh}(X) \cap \mathrm{Q} \operatorname{coh}(X)$.

(b) The injective objects in $\mathrm{Qcoh}(X)$ are precisely the injective objects of $\operatorname{Sh}(X)$ that are quasi-coherent, $\operatorname{InjQ\operatorname {coh}}(X)=\operatorname{InjSh}(X) \cap \mathrm{Qcoh}(X)$.

(c) If $I \in \mathrm{Q} \operatorname{coh}(X)$ is an injective object and $U \subset X$ is open, then $\left.I\right|_{U} \in \mathrm{Q} \operatorname{coh}(U)$ is again injective.

(d) Any direct sum of objects of $\operatorname{Inj} \operatorname{Sh}(X)$ (resp. $\operatorname{InjQcoh}(X)$ ) is in $\operatorname{Inj} \operatorname{Sh}(X)$ (resp. $\operatorname{InjQ} \operatorname{coh}(X))$.

Proof. (a): This is [Har66, Thm. II.7.18].

(b): The inclusion $\supset$ is obvious. For the inclusion $\subset$ let $F \in \operatorname{Qcoh}(X)$. Then $F \subset J$ for $J \in \operatorname{Inj} \operatorname{Sh}(X) \cap \operatorname{Qcoh}(X)$ by (a). If $F$ is injective in $\mathrm{Qcoh}(X)$, this inclusion splits, and hence $F$ is an injective object of $\operatorname{Sh}(X)$.

(c): By (b), $I$ is an injective $\mathcal{O}_{X}$-module. Let $j: U \rightarrow X$ be the inclusion. We have the adjunction $\left(j !, j^{!}=j^{*}\right.$ ) (of functors between $\operatorname{Sh}(X)$ and $\operatorname{Sh}(U)$ ). Since $j$ ! is exact this shows that $j^{*}(I)$ is an injective $\mathcal{O}_{U}$-module. It is quasi-coherent, so we can use (b) again.

(d): The statement for $\operatorname{InjSh}(X)$ is precisely [Har66, Cor. 7.9], and the statement for $\operatorname{InjQ\operatorname {coh}}(X)$ then follows from (b) since the inclusion $\mathrm{Qcoh}(X) \subset \operatorname{Sh}(X)$ preserves direct sums (for Noetherian $X$ one can also use [Har66, Prop. 7.2] and the example before that proposition). 
Definition 2.2. The $d g$ (differential $\mathbb{Z}_{2}$-graded) category $\operatorname{Sh}(X, W)$ is defined as follows. Its objects are $W$-curved $\boldsymbol{d g}$ sheaves on $X$, i. e. diagrams

$$
E=\left(E_{1} \underset{e_{0}}{\stackrel{e_{1}}{\rightleftarrows}} E_{0}\right)
$$

in $\operatorname{Sh}(X)$ satisfying $e_{i+1} e_{i}=W \operatorname{id}_{E_{i}}$, for $i \in \mathbb{Z}_{2}$. The morphism space between two $W$-curved $d g$ sheaves $E, E^{\prime}$ is the graded module

$$
\operatorname{Hom}_{\operatorname{Sh}(X, W)}\left(E, E^{\prime}\right):=\bigoplus_{l \in \mathbb{Z}_{2}}\left(\bigoplus_{i \in \mathbb{Z}_{2}} \operatorname{Hom}_{\mathcal{O}_{X}}\left(E_{i}, E_{i+l}^{\prime}\right)\right)
$$

with differential $d(g)=e^{\prime} \circ g-(-1)^{|g|} g \circ e$ where $g$ is homogeneous of degree $|g|$.

Denote by $\operatorname{Qcoh}(X, W), \operatorname{Coh}(X, W), \operatorname{MF}(X, W), \operatorname{InjQcoh}(X, W), \operatorname{Locfree}(X, W)$, and FlatQcoh $(X, W)$ the full $d g$ subcategories of $\operatorname{Sh}(X, W)$ consisting of objects whose components are quasi-coherent sheaves, coherent sheaves, locally free sheaves of finite type (= vector bundles), injective quasi-coherent sheaves, locally free sheaves, and flat quasi-coherent sheaves, respectively. Objects of $\operatorname{MF}(X, W)$ are called matrix factorizations of $W$.

The shift [1] $E$ of a $W$-curved dg sheaf $E$ as above is defined as

$$
[1] E=\left(E_{0} \underset{-e_{1}}{\stackrel{-e_{0}}{\rightleftarrows}} E_{1}\right) \text {. }
$$

Given a $\operatorname{dg}$ category $\mathcal{C}$, the category $Z_{0}(\mathcal{C})$ and the homotopy category $[\mathcal{C}]$ of $\mathcal{C}$ are defined as usual: they have the same objects as $\mathcal{C}$, but $\operatorname{Hom}_{Z_{0}(\mathcal{C})}\left(E, E^{\prime}\right)=Z_{0}\left(\operatorname{Hom}_{\mathcal{C}}\left(E, E^{\prime}\right)\right)$ and $\operatorname{Hom}_{[\mathcal{C}]}\left(E, E^{\prime}\right)=H_{0}\left(\operatorname{Hom}_{\mathcal{C}}\left(E, E^{\prime}\right)\right)$.

Remark 2.3. The categories $Z_{0}(\operatorname{Sh}(X, W)), Z_{0}(\mathrm{Q} \operatorname{coh}(X, W))$ and $Z_{0}(\operatorname{Coh}(X, W))$ are abelian categories. A sequence in $Z_{0}(\mathrm{MF}(X, W)), Z_{0}(\operatorname{InjQcoh}(X, W)), Z_{0}(\operatorname{Locfree}(X, W))$ or $Z_{0}$ (FlatQcoh $\left.(X, W)\right)$ will be called exact if it is exact in the ambient abelian category $Z_{0}(\mathrm{Q} \operatorname{coh}(X, W))$.

Let $F=\left(\ldots \rightarrow F^{i} \stackrel{d_{F}^{i}}{\longrightarrow} F^{i+1} \rightarrow \ldots\right)$ be a complex in $Z_{0}(\operatorname{Sh}(X, W))$. We define its totalization $\operatorname{Tot}(F)=: T=\left(T_{1} \underset{t_{0}}{\stackrel{t_{1}}{\rightleftarrows}} T_{0}\right) \in \operatorname{Sh}(X, W)$ by

$$
T_{l}:=\bigoplus_{\substack{i \in \mathbb{Z}, j \in \mathbb{Z}_{2}, i+j \equiv l}} F_{j}^{i}
$$

for $l \in \mathbb{Z}_{2}$ and $\left.t_{l}\right|_{F_{j}^{i}}=\left(d_{F}^{i}\right)_{j}+(-1)^{i} f_{j}^{i}$, for $l, j \in \mathbb{Z}_{2}$ and $i \in \mathbb{Z}$ satisfying $i+j \equiv l \bmod 2$.

If $g: E \rightarrow E^{\prime}$ is a morphism in $Z_{0}(\operatorname{Sh}(X, W))$ we define its cone Cone $(g)$ to be the totalization of the complex $\left(\ldots \rightarrow 0 \rightarrow E \stackrel{g}{\rightarrow} E^{\prime} \rightarrow 0 \rightarrow \ldots\right)$ with $E^{\prime}$ in degree zero. This shows that $\operatorname{Sh}(X, W)$ is a pretriangulated dg category, and similarly for $\operatorname{Qcoh}(X, W), \operatorname{Coh}(X, W)$, $\operatorname{MF}(X, W), \operatorname{InjQcoh}(X, W), \operatorname{Locfree}(X, W)$ and FlatQcoh $(X, W)$. In particular, the homotopy categories $[\operatorname{Sh}(X, W)],[\mathrm{Q} \operatorname{coh}(X, W)],[\operatorname{Coh}(X, W)],[\operatorname{MF}(X, W)],[\operatorname{InjQ} \operatorname{coh}(X, W)]$, $[\operatorname{Locfree}(X, W)]$ and $[$ FlatQcoh $(X, W)]$ are triangulated ${ }^{1}$ categories.

\footnotetext{
${ }^{1}$ Our (standard) triangles and the (standard) triangles in [Orl12] differ in the sign of the last morphism. However the associated homotopy categories are equivalent as triangulated categories. For this one may use
} 
Remark 2.4. Notice that one cannot define the cohomology of an object $E \in \operatorname{Sh}(X, W)$ (unless $W=0$ ), but we can define the cohomology of a complex $F$ as above. In particular, it makes sense to ask whether $F$ is exact.

Definition 2.5. Denote by $\operatorname{Acycl}[\operatorname{Sh}(X, W)]$ the full triangulated subcategory of $[\operatorname{Sh}(X, W)]$ classically generated by the totalizations of all short exact sequences

$$
0 \rightarrow F^{1} \rightarrow F^{2} \rightarrow F^{3} \rightarrow 0
$$

with $F^{i} \in \operatorname{Sh}(X, W)$. (Instead of short exact sequences one can take all bounded exact complexes, see Lemma 2.7.(b) below.) By definition, $\operatorname{Acycl}[\operatorname{Sh}(X, W)]$ is a thick subcategory of $[\operatorname{Sh}(X, W)]$, i. e. a strict full triangulated subcategory closed under direct summands.

Following [Pos11b, Pos11a] we define the absolute derived category $\operatorname{DSh}(X, W)$ of $W$-curved $\boldsymbol{d g}$ sheaves as the Verdier quotient

$$
\operatorname{DSh}(X, W):=[\operatorname{Sh}(X, W)] / \operatorname{Acycl}[\operatorname{Sh}(X, W)] .
$$

Similarly, we consider the full subcategories $\operatorname{Acycl}[\mathrm{Qcoh}(X, W)] \subset[\mathrm{Qcoh}(X, W)], \operatorname{Acycl}[\operatorname{Coh}(X, W)] \subset$ $[\operatorname{Coh}(X, W)], \operatorname{Acycl}[M F(X, W)] \subset[M F(X, W)], \operatorname{Acycl}[\operatorname{Locfree}(X, W)] \subset[\operatorname{Locfree}(X, W)]$, $\operatorname{Acycl}[\operatorname{FlatQ} \operatorname{coh}(X, W)] \subset[\operatorname{FlatQ} \operatorname{coh}(X, W)]$, and the corresponding Verdier quotients

$$
\begin{aligned}
\operatorname{DQcoh}(X, W) & =[\mathrm{Qcoh}(X, W)] / \operatorname{Acycl}[\mathrm{Qcoh}(X, W)], \\
\operatorname{DCoh}(X, W) & =[\operatorname{Coh}(X, W)] / \operatorname{Acycl}[\operatorname{Coh}(X, W)], \\
\operatorname{MF}(X, W) & =[\operatorname{MF}(X, W)] / \operatorname{Acycl}[\operatorname{MF}(X, W)], \\
\operatorname{DLocfree}(X, W) & =[\operatorname{Locfree}(X, W)] / \operatorname{Acycl}[\operatorname{Locfree}(X, W)], \\
\operatorname{DFlatQcoh}(X, W) & =[\operatorname{FlatQcoh}(X, W)] / \operatorname{Acycl}[\operatorname{FlatQcoh}(X, W)] .
\end{aligned}
$$

The triangulated category $\mathrm{MF}(X, W)$ is called the category of matrix factorizations of $W$.

There is another characterization of $\operatorname{Acycl}[\operatorname{MF}(X, W)]$ given in Corollary 2.59 below. We will be mainly interested in the category $\mathbf{M F}(X, W)$.

Remark 2.6. Let $X_{1}, \ldots, X_{m}$ be the connected components of $X$. Then

$$
\operatorname{DSh}(X, W)=\prod_{i=1}^{m} \operatorname{DSh}\left(X_{i}, W\right),
$$

and similarly for all other categories defined above. So to study these categories one may assume that $X$ is connected (if needed), and then the map $W$ is either flat or else constant (here constant means that $W(X)$ consists of a single point in $\mathbb{A}^{1}$ which is then necessarily closed; if we think of $W$ as an element of $\Gamma\left(X, \mathcal{O}_{X}\right)$ it means that $W \in k \subset \Gamma\left(X, \mathcal{O}_{X}\right)$ ).

Here is a useful lemma.

Lemma 2.7. Let $\mathcal{M}$ be $\operatorname{Sh}(X, W), \operatorname{Qcoh}(X, W), \operatorname{Coh}(X, W), \operatorname{MF}(X, W), \operatorname{Locfree}(X, W)$, or FlatQcoh $(X, W)$.

[KS94, 10.1.10.i] or the equivalence that multiplies the differentials $e_{0}, e_{1}$ of all objects $E$ by -1 and is the identity on morphisms. 
(a) Any short exact sequence $0 \rightarrow F^{-1} \stackrel{p}{\rightarrow} F^{0} \stackrel{q}{\rightarrow} F^{1} \rightarrow 0$ in $Z_{0}(\mathcal{M})$ gives rise to a triangle $F^{-1} \stackrel{p}{\rightarrow} F^{0} \stackrel{q}{\rightarrow} F^{1} \rightarrow[1] F^{-1}$ in $\mathrm{D} \mathcal{M}$ (where $\operatorname{D~MF}(X, W):=\operatorname{MF}(X, W)$ ).

(b) Let $F=\left(\ldots \rightarrow 0 \rightarrow F^{1} \stackrel{f^{1}}{\rightarrow} F^{2} \stackrel{f^{2}}{\rightarrow} F^{3} \stackrel{f^{3}}{\rightarrow} F^{4} \rightarrow \cdots \rightarrow F^{n} \rightarrow 0 \rightarrow \ldots\right)$ be a bounded exact complex in $Z_{0}(\mathcal{M})$. Then $\operatorname{Tot}(F) \in \operatorname{Acycl}[\mathcal{M}]$.

(c) If $F=\left(\ldots \rightarrow 0 \rightarrow P^{a} \rightarrow \ldots \rightarrow P^{b} \stackrel{v}{\rightarrow} I^{b+1} \rightarrow \ldots \rightarrow I^{c} \rightarrow 0 \rightarrow \ldots\right)$ is a bounded complex in $Z_{0}(\mathcal{M})$ that is composed of two bounded complexes $P$ and $I$ as indicated, there is a standard triangle

$$
[1] \operatorname{Tot}(P) \stackrel{v}{\rightarrow} \operatorname{Tot}(I) \rightarrow \operatorname{Tot}(F) \rightarrow \operatorname{Tot}(P)
$$

in $[\mathcal{M}]$. If $F$ is exact, $[1] \operatorname{Tot}(P) \stackrel{v}{\rightarrow} \operatorname{Tot}(I)$ is an isomorphism in $\mathrm{D} \mathcal{M}$.

(d) Let $F$ be a bounded complex in $Z_{0}(\mathcal{M})$. If each $F^{i}$ is isomorphic to 0 in $[\mathcal{M}]$, then $\operatorname{Tot}(F)=0$ in $[\mathcal{M}]$. Similarly, if each $F^{i}$ is in $\operatorname{Acycl}[\mathcal{M}]$, then $\operatorname{Tot}(F) \in \operatorname{Acycl}[\mathcal{M}]$.

Proof. (a): We have standard triangles

$$
F^{-1} \stackrel{p}{\rightarrow} F^{0} \stackrel{\left[\begin{array}{l}
1 \\
0
\end{array}\right]}{\longrightarrow} \operatorname{Cone}(p) \stackrel{[01]}{\longrightarrow}[1] F^{-1}
$$

where $\operatorname{Cone}(p)=F^{0} \oplus[1] F^{-1}$ as a graded sheaf, and

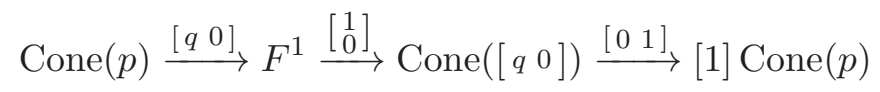

in $[\mathcal{M}]$. Note that

$$
\operatorname{Cone}\left(\left[\begin{array}{ll}
q & 0
\end{array}\right]\right)=F^{1} \oplus[1] \operatorname{Cone}(p)=F^{1} \oplus[1] F^{0} \oplus F^{-1}
$$

has differential $\left[\begin{array}{ccc}f^{1} & q & 0 \\ 0 & -f^{0} & -p \\ 0 & 0 & f^{-1}\end{array}\right]$ and hence is the totalization of the exact complex $0 \rightarrow F^{-1} \longrightarrow$ $F^{0} \stackrel{q}{\rightarrow} F^{1} \rightarrow 0$ with $F^{0}$ in odd degree. This implies that Cone $(p) \stackrel{[q 0]}{\longrightarrow} F^{1}$ becomes an isomorphism in $\mathrm{D} \mathcal{M}$.

(b): Factor $F^{2} \rightarrow F^{3}$ in $Z_{0}(\operatorname{Sh}(X, W))$ into an epimorphism followed by a monomorphism, $F^{2} \stackrel{p}{\rightarrow} Q \stackrel{i}{\rightarrow} F^{3}$, and note that $Q \in \mathcal{M}$ (for example, if $\mathcal{M}=\mathrm{MF}(X, W)$, the kernel of $F^{n-1} \rightarrow F^{n}$ is in $\operatorname{MF}(X, W)$, and we can iterate this argument). Consider the vertical morphism of horizontal complexes

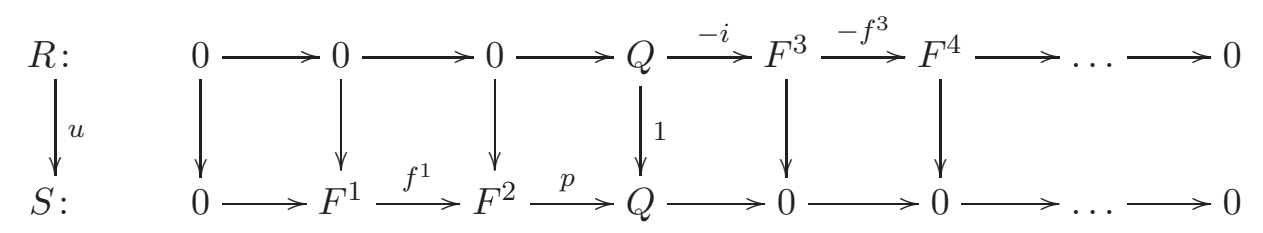

We leave it to the reader to check that the mapping cone Cone $(u)$ of this morphism is isomorphic to $F$ in the homotopy category of complexes in $Z_{0}(\mathcal{M})$. Hence $\operatorname{Tot}(\operatorname{Cone}(u)) \cong \operatorname{Tot}(F)$ in $[\mathcal{M}]$. On the other hand we have a short exact sequence $0 \rightarrow \operatorname{Tot}(S) \rightarrow \operatorname{Tot}(\operatorname{Cone}(u)) \rightarrow$ [1] $\operatorname{Tot}(R) \rightarrow 0$ in $Z_{0}(\mathcal{M})$ and hence a triangle $\operatorname{Tot}(S) \rightarrow \operatorname{Tot}(\operatorname{Cone}(u)) \rightarrow[1] \operatorname{Tot}(R) \rightarrow$ [1] $\operatorname{Tot}(S)$ in $\mathrm{D} \mathcal{M}$ by (a). By induction $\operatorname{Tot}(S)$ and $\operatorname{Tot}(R)$ are in $\operatorname{Acycl}[\mathcal{M}]$, and hence $\operatorname{Tot}(F) \cong \operatorname{Tot}(\operatorname{Cone}(u)) \in \operatorname{Acycl}[\mathcal{M}]$.

(c): Obvious. If $F$ is exact, use (b). 
(d): We argue by induction on the number of $i$ with $F^{i} \neq 0$ in $\mathcal{M}$. If this number is $\leq 1$ the claim is obvious. Otherwise let $i \in \mathbb{Z}$ be non-maximal with $F^{i} \neq 0$. Let $w_{\leq i} F$ be the complex obtained from $F$ by replacing all terms in degrees $>i$ by 0 , and define $w_{>i} F$ similarly. As in (c), there is a standard triangle

$$
[1] \operatorname{Tot}\left(w_{\leq i} F\right) \rightarrow \operatorname{Tot}\left(w_{>i} F\right) \rightarrow \operatorname{Tot}(F) \rightarrow \operatorname{Tot}\left(w_{\leq i} F\right)
$$

in $[\mathcal{M}]$. By induction the first two terms are isomorphic to zero in $[\mathcal{M}]$ (resp. are in $\operatorname{Acycl}[\mathcal{M}])$, hence so is $\operatorname{Tot}(F)$.

2.2. Matrix factorizations and the category of singularities. In case the morphism $W: X \rightarrow \mathbb{A}^{1}$ is flat we recall an important theorem proved in [Orl12]. Recall that the category of singularities $D_{\mathrm{Sg}}(Y)$ of a Noetherian scheme $Y$ is defined as the Verdier quotient

$$
D_{\mathrm{Sg}}(Y):=D^{b}(\operatorname{Coh}(Y)) / \mathfrak{P e r f}(Y),
$$

where $D^{b}(\operatorname{Coh}(Y))$ is the bounded derived category of coherent sheaves on $Y$ and $\mathfrak{P e r f}(Y)$ is the category of perfect complexes.

Let $X_{0}$ be the scheme-theoretical zero fiber of the morphism $W: X \rightarrow \mathbb{A}^{1}$. Given $E=$ $\left(E_{1} \underset{e_{0}}{\stackrel{e_{1}}{\rightleftarrows}} E_{0}\right) \in \mathbf{M F}(X, W)$ the cokernel of the map $e_{1}$ is annihilated by $W$, hence it comes from an object in $\operatorname{Coh}\left(X_{0}\right)$. We denote this object by cok $e_{1}$.

Theorem 2.8 ([Orl12]). Assume that the morphism $W: X \rightarrow \mathbb{A}^{1}$ is flat. Then the above construction induces a functor

$$
\text { cok: } \mathbf{M F}(X, W) \rightarrow D_{\mathrm{Sg}}\left(X_{0}\right)
$$

which is an equivalence of triangulated categories.

The above theorem is useful because it gives us two completely different descriptions of the same triangulated category.

2.3. Some embeddings and equivalences. Our next aim is to prove the equivalences and embeddings stated in the following theorem.

\section{Theorem 2.9.}

(a) The functor $[\operatorname{InjQ} \operatorname{coh}(X, W)] \rightarrow \operatorname{DQ} \operatorname{coh}(X, W)$ is an equivalence.

(b) The functor $\operatorname{DCoh}(X, W) \rightarrow \operatorname{DQcoh}(X, W)$ is full and faithful.

(c) The functor $\operatorname{MF}(X, W) \rightarrow \operatorname{DCoh}(X, W)$ is an equivalence.

(d) The functor $\operatorname{DLocfree}(X, W) \rightarrow \mathrm{DQ} \operatorname{coh}(X, W)$ is an equivalence.

(e) The functor DFlatQcoh $(X, W) \rightarrow \mathrm{DQ} \operatorname{coh}(X, W)$ is an equivalence.

Proof. Consider the commutative diagrams of inclusions of full triangulated categories

$\begin{array}{cccccccc}\operatorname{Acycl}[\operatorname{MF}(X, W)] & \subset & \operatorname{Acycl}[\operatorname{Coh}(X, W)] & \subset & \operatorname{Acycl}[\mathrm{Qcoh}(X, W)] & \supset & \{0\} \\ \cap & (\text { 困 } 1) & \cap & (\text { 米2) } & \cap & (\text { 困3) } & \cap \\ {[\operatorname{MF}(X, W)]} & \subset & {[\operatorname{Coh}(X, W)]} & \subset & {[\mathrm{Qcoh}(X, W)]} & \supset & {[\operatorname{InjQ} \operatorname{coh}(X, W)]}\end{array}$


and

$$
\begin{array}{cccccc}
\operatorname{Acycl}[\operatorname{Locfree}(X, W)] & \subset & \operatorname{Acycl}[\mathrm{Qcoh}(X, W)] & \supset & \operatorname{Acycl}[\operatorname{FlatQcoh}(X, W)] \\
\cap & (\text { 困4) } & \cap & (\text { 困5) } & \cap \\
{[\operatorname{Locfree}(X, W)]} & \subset & {[\mathrm{Qcoh}(X, W)]} & \supset & {[\operatorname{FlatQcoh}(X, W)] .}
\end{array}
$$

We will show that the three equivalent conditions (ff1) ${ }^{\mathrm{op}}$, (ff2 $)^{\mathrm{op}}$, (ff3) ${ }^{\mathrm{op}}$ of Proposition B.2 hold for the squares (困1) and (困2) (and then also for the rectangle formed out of these two squares), and for the squares (因) and (因5), and that the three equivalent conditions (ff1), (ff2), (ff3) hold for the square (困3). This will imply that all five functors in Theorem 2.9 are full and faithful.

The following lemma is essentially contained in [Pos11b, Thm. 3.6]. It shows that the functors considered in parts (a), (c), (d) and (e) of Theorem 2.9 are essentially surjective.

\section{Lemma 2.10.}

(a) For any $F \in \mathrm{Q} \operatorname{coh}(X, W)$ there exists an exact sequence $0 \rightarrow F \rightarrow I^{0} \rightarrow \cdots \rightarrow$ $I^{n} \rightarrow 0$ in $Z_{0}(\mathrm{Q} \operatorname{coh}(X, W))$ with all $I^{j} \in \operatorname{InjQ} \operatorname{coh}(X, W)$. In particular, the obvious morphism $F \rightarrow \operatorname{Tot}(I)$ has its cone in $\operatorname{Acycl}[\mathrm{Q} \operatorname{coh}(X, W)]$ and hence becomes an isomorphism in $\mathrm{DQ} \operatorname{coh}(X, W)$.

(b) Let $E \in \operatorname{Coh}(X, W)$. Then there exists an exact sequence $0 \rightarrow P^{n} \rightarrow \cdots \rightarrow P^{0} \rightarrow$ $E \rightarrow 0$ in $Z_{0}(\operatorname{Coh}(X, W))$ with all $P^{i} \in \operatorname{MF}(X, W)$. In particular, the obvious morphism $\operatorname{Tot}(P) \rightarrow E$ has its cone in $\operatorname{Acycl}[\operatorname{Coh}(X, W)]$ and hence becomes an isomorphism in $\mathrm{DCoh}(X, W)$.

(c) Let $E \in \mathrm{Q} \operatorname{coh}(X, W)$. Then there exists an exact sequence $0 \rightarrow P^{n} \rightarrow \cdots \rightarrow P^{0} \rightarrow$ $E \rightarrow 0$ in $Z_{0}(\operatorname{Qcoh}(X, W))$ with all $P^{i} \in \operatorname{Locfree}(X, W) \subset \operatorname{FlatQcoh}(X, W)$. In particular, the obvious morphism $\operatorname{Tot}(P) \rightarrow E$ has its cone in $\operatorname{Acycl}[\mathrm{Qcoh}(X, W)]$ and hence becomes an isomorphism in $\mathrm{DQcoh}(X, W)$.

Proof. (a) Choose injective morphisms $g_{0}: F_{0} \rightarrow J_{0}$ and $g_{1}: F_{1} \rightarrow J_{1}$, such that $J_{0}$ and $J_{1}$ are injective quasi-coherent sheaves (use Theorem 2.1). Consider the object $I^{\prime} \in \operatorname{InjQcoh}(X, W)$, where $I_{0}^{\prime}=I_{1}^{\prime}=J_{0} \oplus J_{1}$ and $i_{0}^{\prime}=W \oplus \mathrm{id}, i_{1}^{\prime}=\mathrm{id} \oplus W$. We denote $I^{\prime}$ by $G^{-}(J)$ for future reference. Note that $G^{-}(J)$ only depends on the graded sheaf $J$.

Let $h=\left(h_{0}, h_{1}\right): F \rightarrow I^{\prime}$ be the injective morphism in $Z_{0}\left(\mathrm{Q} \operatorname{coh}(X, W)\right.$ given by $h_{0}=$ $\left(g_{0}, g_{1} f_{0}\right)^{\mathrm{t}}, h_{1}=\left(g_{0} f_{1}, g_{1}\right)^{\mathrm{t}}$. Now define $I^{0}:=I^{\prime}$, replace $F$ by cok $h$ and repeat the procedure. Since $X$ is regular of finite Krull dimension we eventually arrive at the desired finite resolution (the global dimension of all local rings $\mathcal{O}_{X, x}$ is bounded by the Krull dimension of $X$, and injectivity of a quasi-coherent sheaf can be tested on the stalks by [Har66, Prop. 7.17] and Theorem 2.1). The isomorphism $F \stackrel{\sim}{\rightarrow} \operatorname{Tot}(I)$ in $\operatorname{DQcoh}(X, W)$ follows from Lemma 2.7.(c).

(b) We apply the dual process. Namely, our assumptions on $X$ allow us to choose vector bundles $N_{0}$ and $N_{1}$ with surjective morphisms $g_{0}: N_{0} \rightarrow E_{0}$ and $g_{1}: N_{1} \rightarrow E_{1}$. Consider the object $P^{\prime} \in \operatorname{MF}(X, W)$ where $P_{0}^{\prime}=P_{1}^{\prime}=N_{0} \oplus N_{1}$ and $p_{0}^{\prime}=\mathrm{id} \oplus W, p_{1}^{\prime}=W \oplus \mathrm{id}$. We denote $P^{\prime}$ by $G^{+}(N)$ for future reference. It only depends on the graded sheaf $N$. 
Let $h: P^{\prime} \rightarrow E$ be the surjective morphism in $Z_{0}(\operatorname{Coh}(X, W))$ given by $h_{0}=\left(g_{0}, e_{1} g_{1}\right)$, $h_{1}=\left(e_{0} g_{0}, g_{1}\right)$. Now replace $E$ by ker $h$ and repeat the procedure. Since $X$ is regular of finite Krull dimension we eventually arrive at the desired finite resolution. The last statement follows from Lemma 2.7.(c).

(c): Since any quasi-coherent sheaf is the union of its coherent subsheaves ([Har77, Exercise II.5.15(e)]) there are locally free sheaves $N_{0}$ and $N_{1}$ with epimorphisms $g_{i}: N_{i} \rightarrow E_{i}$. We then proceed as in the proof of (b), using [Bas63, Corollary 4.5].

Remark 2.11. If $K$ is any quasi-coherent sheaf on $X$, then $(K \underset{W}{\stackrel{\mathrm{id}}{\rightleftarrows}} K) \in \mathrm{Q} \operatorname{coh}(X, W)$ is obviously zero in $[\mathrm{Q} \operatorname{coh}(X, W)]$ and in $\mathrm{DQ} \operatorname{coh}(X, W)$. So if $0 \rightarrow F \rightarrow I^{0} \rightarrow \cdots \rightarrow I^{n} \rightarrow 0$ is the exact sequence constructed in the proof of Lemma 2.10.(a), all objects $I^{0}, \ldots, I^{n-1}$ are zero in $\mathrm{DQcoh}(X, W)$. This implies that $F$ and $[n] I^{n}$ are isomorphic in $\operatorname{DQcoh}(X, W)$ (use Lemma 2.7.(a)). Similar conclusions hold for the exact sequences constructed in the proof of parts (b) and (c) of Lemma 2.10.

Remark 2.12. Let $p: E \rightarrow F$ be a morphism in $Z_{0}(Q \operatorname{coh}(X, W))$, and let $0 \rightarrow E \rightarrow A^{0} \rightarrow$ $A^{1} \rightarrow \ldots$ be an exact sequence in $Z_{0}(\mathrm{Q} \operatorname{coh}(X, W))$. Then there is a resolution $F \rightarrow I$ as in Lemma 2.10.(a) and a morphism $A \rightarrow I$ of resolutions that lifts $p$.

Namely, in the notation of the proof of Lemma 2.10.(a), find morphisms $q_{l}: A_{l}^{0} \rightarrow J_{l}$ that restrict to $g_{l} p_{l}$ on $E_{l}$, for $l=0,1$. Then $\left(q_{0}, q_{1} a_{0}\right)^{\mathrm{t}}: A_{0}^{0} \rightarrow I_{0}^{0}=J_{0} \oplus J_{1}$ and $\left(q_{0} a_{1}, q_{1}\right)^{\mathrm{t}}: A_{1}^{0} \rightarrow$ $I_{1}^{0}=J_{0} \oplus J_{1}$ define a morphism $A^{0} \rightarrow I^{0}$ that lifts $p: E \rightarrow F$. Pass to the cokernels and proceed.

Lemma 2.13. We have

$$
\operatorname{Hom}_{[\mathrm{Qcoh}(X, W)]}(\operatorname{Acycl}[\mathrm{Q} \operatorname{coh}(X, W)],[\operatorname{InjQ} \operatorname{coh}(X, W)])=0 .
$$

In particular, condition (ff2) of Proposition B.2 holds for the square (困3).

Proof. Let $J \in \operatorname{InjQ\operatorname {coh}}(X, W)$. Let $0 \rightarrow E^{1} \rightarrow E^{2} \rightarrow E^{3} \rightarrow 0$ be a short exact sequence in $Z_{0}(\mathrm{Q} \operatorname{coh}(X, W))$ with totalization $\operatorname{Tot}(E)$. The dg module $\operatorname{Hom}_{\mathrm{Qcoh}(X, W)}(\operatorname{Tot}(E), J)$ is the totalization of the short exact sequence

$$
0 \rightarrow \operatorname{Hom}_{\mathrm{Q} c o h(X, W)}\left(E^{3}, J\right) \rightarrow \operatorname{Hom}_{\mathrm{Q} c o h(X, W)}\left(E^{2}, J\right) \rightarrow \operatorname{Hom}_{\mathrm{Q} c o h(X, W)}\left(E^{1}, J\right) \rightarrow 0
$$

of dg modules. Hence it is obviously (or by Lemma 2.46 below) acyclic, so $\operatorname{Hom}_{[\mathrm{Qcoh}(X, W)]}(\operatorname{Tot}(E), J)=$ 0 . This implies the lemma.

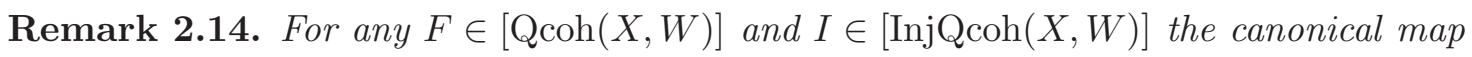

$$
\operatorname{Hom}_{[\mathrm{Qcoh}(X, W)]}(F, I) \stackrel{\sim}{\rightarrow} \operatorname{Hom}_{\mathrm{DQ} \operatorname{coh}(X, W)}(F, I)
$$

is an isomorphism, since condition (ff3) holds for the square (困3).

Lemma 2.15. Condition (ff2) of ${ }^{\text {op }}$ Proposition B.2 holds for the square (困2). Namely, let $L \in[\operatorname{Coh}(X, W)]$ and $A \in \operatorname{Acycl}[\mathrm{Q} \operatorname{coh}(X, W)]$. Then any morphism $L \rightarrow A$ in $[\mathrm{Qcoh}(X, W)]$ factors through some object $A^{\prime} \in \operatorname{Acycl}[\operatorname{Coh}(X, W)]$. 
Proof. Step 1: Let $E=\left(E_{1} \underset{e_{0}}{\stackrel{e_{1}}{\rightleftarrows}} E_{0}\right) \in \operatorname{Qcoh}(X, W)$ and let $K \subset E$ be a graded coherent subsheaf, i. e. $K_{i} \subset E_{i}$ is a coherent subsheaf for $i=0,1$. Then there exists $F \in \operatorname{Coh}(X, W)$ such that $F \subset E$ in $Z_{0}(\mathrm{Q} \operatorname{coh}(X, W))$ and $K \subset F$ as graded sheaves. Indeed, take $F_{1}=$ $K_{1}+e_{0} K_{0}, F_{0}=K_{0}+e_{1} K_{1}$.

Step 2: Given an exact sequence

$$
E=\left(0 \rightarrow E^{1} \stackrel{d^{1}}{\rightarrow} E^{2} \rightarrow \cdots \rightarrow E^{n-1} \stackrel{d^{n-1}}{\longrightarrow} E^{n} \rightarrow 0\right)
$$

in $Z_{0}(\mathrm{Q} \operatorname{coh}(X, W))$ and graded coherent subsheaves $K^{i} \subset E^{i}$, there exists an exact sequence

$$
0 \rightarrow F^{1} \rightarrow \cdots \rightarrow F^{n} \rightarrow 0
$$

in $Z_{0}(\operatorname{Coh}(X, W))$ which is a subsequence of $E$ such that $K^{i} \subset F^{i}$ for all $i=1, \ldots, n$.

Indeed, first we may assume that $d^{i}\left(K^{i}\right) \subset K^{i+1}$ (by replacing $K^{i+1}$ with $K^{i+1}+d^{i}\left(K^{i}\right)$ ). Using Step 1, we find a subobject $F^{n} \subset E^{n}$, such that $F^{n} \in \operatorname{Coh}(X, W)$ and $K^{n} \subset F^{n}$. Between $K^{n-1}$ and $\left(d^{n-1}\right)^{-1}\left(F^{n}\right)$ there is a graded coherent sheaf surjecting onto $F^{n}$ (use [Har77, Ex. II.5.15]). Step 1 again then shows that there is an object $F^{n-1} \in \operatorname{Coh}(X, W)$ such that $K^{n-1} \subset F^{n-1} \subset E^{n-1}$ and $d^{n-1}\left(F^{n-1}\right)=F^{n}$.

Now proceed by induction with $F^{n-1} \cap \operatorname{ker} d^{n-1} \subset \operatorname{ker} d^{n-1}$ instead of $F^{n} \subset E^{n}$ and note that $d^{n-2}\left(K^{n-2}\right) \subset K^{n-1} \cap \operatorname{ker} d^{n-1}$.

Step 3: Assume that $A=\operatorname{Tot}(E)$ is the totalization of an exact sequence $E$ as above and let $g: L \rightarrow A$ be a morphism in [Qcoh $(X, W)]$. Represent $g$ by a morphism $\hat{g}: L \rightarrow A$ in $Z_{0}(\mathrm{Q} \operatorname{coh}(X, W)]$ and let $K \subset A$ be the image of $\hat{g}$. Let $K_{l}^{i}$ be the image of $K_{i+l}$ under the obvious projection $\operatorname{Tot}(E)_{i+l} \rightarrow E_{l}^{i}$ of sheaves. This defines the graded sheaves $K^{i}$. Step 2 applied in this setting yields an exact sequence $F$ in $Z_{0}(\operatorname{Coh}(X, W))$ such that $\hat{g}$ factors through $A^{\prime}=\operatorname{Tot}(F) \subset A$. Hence $g$ factors through $A^{\prime} \in \operatorname{Acycl}[\operatorname{Coh}(X, W)]$.

Now use that condition (ff4) ${ }^{\text {op }}$ in Proposition B.2 implies condition (ff2) ${ }^{\text {op }}$ (it would have been sufficient to consider totalizations of short exact sequences only). This finishes the proof.

Lemma 2.16. The three equivalent conditions (ff1) $)^{\mathrm{op}}$, (ff2) $)^{\mathrm{op}}$, (ff3) ${ }^{\mathrm{op}}$ hold for the square (困1).

We will give two proofs of this key fact. The first proof from [LP11] is short but uses Theorem 2.8 and hence only works in case the morphism $W: X \rightarrow \mathbb{A}^{1}$ is flat. The second proof is essentially the one given in [Pos11a, Prop. 1.5] (in a slightly different language) and works in general.

Lemma 2.17. Assume that $X$ is affine.

(a) Then

$$
\operatorname{Hom}_{[Q \operatorname{coh}(X, W)]}([\operatorname{MF}(X, W)], \operatorname{Acycl}[\mathrm{Q} \operatorname{coh}(X, W)])=0 .
$$

In particular, $[\mathrm{MF}(X, W)] \cap \operatorname{Acycl}[\mathrm{Qcoh}(X, W)]=0,[\mathrm{MF}(X, W)] \cap \operatorname{Acycl}[\operatorname{Coh}(X, W)]=$ 0 and $\operatorname{Acycl}[\operatorname{MF}(X, W)]=0$.

(b) $[\mathrm{MF}(X, W)] \stackrel{\sim}{\rightarrow} \mathbf{M F}(X, W)$ canonically. 
Proof. Clearly (a) implies (b). To prove (a) we argue as in the proof of Lemma 2.13. Namely let $P \in \operatorname{MF}(X, W)$ and let $E$ be the totalization of a short exact sequence

$$
0 \rightarrow E^{1} \rightarrow E^{2} \rightarrow E^{3} \rightarrow 0
$$

in $Z_{0}(\mathrm{Q} \operatorname{coh}(X, W))$. Then the dg module $\operatorname{Hom}_{\mathrm{Q} \operatorname{coh}(X, W)}(P, E)$ is the totalization of the short exact (since $X$ is affine we can view both $P_{i}$ as projective $\Gamma\left(X, \mathcal{O}_{X}\right)$-modules) sequence

$$
0 \rightarrow \operatorname{Hom}_{\mathrm{Q} c o h}(X, W)\left(P, E^{1}\right) \rightarrow \operatorname{Hom}_{\mathrm{Q} c o h}(X, W)\left(P, E^{2}\right) \rightarrow \operatorname{Hom}_{\mathrm{Qcoh}(X, W)}\left(P, E^{3}\right) \rightarrow 0
$$

of dg modules and hence is acyclic. This implies all the assertions in (a).

Proof of Lemma 2.16 in case $W: X \rightarrow \mathbb{A}^{1}$ is flat. We show that condition (ff1) op holds for the square (困1): Let $s: E \rightarrow P$ in $[\operatorname{Coh}(X, W)]$ with $P \in[\operatorname{MF}(X, W)]$ and cone in $\operatorname{Acycl}[\operatorname{Coh}(X, W)]$. Then there exists $t: P^{\prime} \rightarrow E$ with $P^{\prime} \in[\operatorname{MF}(X, W)]$ such that the cone of st is in $\operatorname{Acycl}[\operatorname{MF}(X, W)]$.

By Lemma 2.10.(b) we can find $t$ and $P^{\prime}$ as required such that the cone of $t$ is in $\operatorname{Acycl}[\operatorname{Coh}(X, W)]$. Hence the cone of $s t$ is in $\operatorname{Acycl}[\operatorname{Coh}(X, W)]$, and obviously in $[\operatorname{MF}(X, W)]$. We need to show that $[\operatorname{MF}(X, W)] \cap \operatorname{Acycl}[\operatorname{Coh}(X, W)]=\operatorname{Acycl}[\operatorname{MF}(X, W)]$. Namely, let $F \in[\mathrm{MF}(X, W)] \cap \operatorname{Acycl}[\operatorname{Coh}(X, W)]$. It suffices to show that its image cok $F$ in $D_{\mathrm{Sg}}\left(X_{0}\right)$ under the equivalence of Theorem 2.8 is zero. But this is true locally by Lemma 2.17, and hence globally.

Proof of Lemma 2.16 for arbitrary $W: X \rightarrow \mathbb{A}^{1}$. It suffices to prove the following claim (use condition (ff4) ${ }^{\text {op }}$ of Proposition B.2): Let $E \in[\mathrm{MF}(X, W)]$ and let $L$ be the totalization of a short exact sequence

$$
0 \rightarrow U \stackrel{i}{\rightarrow} V \stackrel{p}{\rightarrow} Q \rightarrow 0
$$

in $Z_{0}(\operatorname{Coh}(X, W))$ (with $U$ of odd degree). Then any morphism $E \rightarrow L$ in $[\operatorname{Coh}(X, W)]$ factors through an object of $\operatorname{Acycl}[\operatorname{MF}(X, W)]$.

Step 1: Let $G \in \operatorname{Coh}(X, W)$. Let $\gamma: G \rightarrow L$ be a degree zero morphism in $\operatorname{Coh}(X, W)$. Then $\gamma=(a, b, c)$ where $a: G \rightarrow U, b: G \rightarrow V$ and $c: G \rightarrow Q$ are morphisms in $\operatorname{Coh}(X, W)$ of degrees 1, 0, 1, respectively. Notice that the differential of $\gamma$ is given by the formula $d \gamma=d(a, b, c)=(-d a, i a+d b, p b-d c)$.

Lemma 2.18. In this setting assume that the degree zero morphism $\gamma=(a, b, c): G \rightarrow L$ is closed and that $c$ can be lifted to a degree one morphism $t: G \rightarrow V$ in $\operatorname{Coh}(X, W)$, i. e. $p t=c$. Then $\gamma$ is homotopic to zero.

Proof. Let Hom $=\operatorname{Hom}_{\mathrm{Coh}(X, W)}$. We have an exact sequence of dg modules

$$
0 \rightarrow \operatorname{Hom}(G, U) \stackrel{i_{*}}{\rightarrow} \operatorname{Hom}(G, V) \stackrel{p_{*}}{\rightarrow} \operatorname{Hom}(G, Q) .
$$

Note that $d c=d(p t)=p d t$. Then $p(b-d t)=p b-d c=0$, so there exists a degree zero morphism $s \in \operatorname{Hom}(G, U)$ such that $b-d t=i s$. Then $i d s=d(i s)=d b=-i a$, hence $-d s=a$ and $d(s, t, 0)=(a, b, c)=\gamma$.

Step 2: Now assume that $N$ is a graded coherent sheaf. Recall the object $G^{+}(N) \in$ $\operatorname{Coh}(X, W)$ freely generated by $N$ (see the proof of Lemma 2.10 above) and note that there is a canonical inclusion $N \subset G^{+}(N)$ of graded sheaves. For any $S \in \operatorname{Coh}(X, W)$ a degree 
zero morphism $r: G^{+}(N) \rightarrow S$ is uniquely determined by the restrictions $\left.r\right|_{N}$ and $\left.(d r)\right|_{N}$; conversely, given two graded morphisms $N \rightarrow S$ of degrees 0 and 1 respectively, they arise from such a morphism $r$. A similar statement holds for degree one morphisms $G^{+}(N) \rightarrow S$.

Let $\nu: N \rightarrow L$ be a degree zero morphism of graded sheaves. Similarly as above we represent it as a triple $\nu=\left(a^{\prime}, b^{\prime}, c^{\prime}\right)$ where $a^{\prime}: N \rightarrow U, b^{\prime}: N \rightarrow V$ and $c^{\prime}: N \rightarrow Q$ are morphisms of graded sheaves of degrees $1,0,1$, respectively.

Lemma 2.19. In this setting assume that the degree one morphism $c^{\prime}: N \rightarrow Q$ of graded sheaves can be lifted to a degree one morphism $s: N \rightarrow V$, i. e. ps $=c^{\prime}$. Let $\widetilde{\nu}: G^{+}(N) \rightarrow L$ be the closed degree zero morphism uniquely determined by $\left.\widetilde{\nu}\right|_{N}=\nu: N \rightarrow L$ (and $d \widetilde{\nu}=0$ ), and let $\widetilde{\nu}=(a, b, c)$ be its components. Then the degree one morphism $c: G^{+}(N) \rightarrow Q$ can be lifted to a degree one morphism $t: G^{+}(N) \rightarrow V$, i. e. $p t=c$.

Proof. Extend the degree one morphism $s: N \rightarrow V$ to a unique degree one morphism $t: G^{+}(N) \rightarrow V$ such that $\left.(d t)\right|_{N}=b^{\prime}$. Note that $\left.\widetilde{\nu}\right|_{N}=\nu$ implies $\left.b\right|_{N}=b^{\prime}$ and $\left.c\right|_{N}=c^{\prime}$, and that $d \widetilde{\nu}=0$ implies $p b=d c$. So $\left.p t\right|_{N}=p s=c^{\prime}=\left.c\right|_{N}$ and $\left.(d(p t))\right|_{N}=\left.p(d t)\right|_{N}=p b^{\prime}=$ $\left.p b\right|_{N}=\left.(d c)\right|_{N}$. Hence $p t=c$.

Step 3: To complete the proof assume that we are given a morphism $E \rightarrow L$ in $[\operatorname{Coh}(X, W)]$, which we represent by a closed degree zero morphism morphism $\varepsilon=\left(a^{\prime \prime}, b^{\prime \prime}, c^{\prime \prime}\right): E \rightarrow$ $L$ where $a^{\prime \prime}, b^{\prime \prime}, c^{\prime \prime}$ are as explained above. Let $N$ be a graded vector bundle mapping surjectively onto the "fiber product" $V \times{ }_{Q} E$ of the morphisms $p: V \rightarrow Q$ and $c^{\prime \prime}: E \rightarrow Q$ (for $l \in \mathbb{Z}_{2}$ we have $\left.\left(V \times{ }_{Q} E\right)_{l}=V_{l+1} \times{ }_{Q_{l+1}} E_{l}\right)$. This yields a surjective degree zero morphism $q: N \rightarrow E$ of graded sheaves such that $c^{\prime \prime} q: N \rightarrow Q$ can be lifted to $V$.

Let $\nu:=\varepsilon q: N \rightarrow L$; its third component is $c^{\prime}:=c^{\prime \prime} q$. Let $\widetilde{\nu}=(a, b, c): G^{+}(N) \rightarrow L$ be the closed degree zero extension of $\nu$. By Lemma 2.19 the morphism $c$ can be lifted to a degree one morphism $t: G^{+}(N) \rightarrow V$, i. e. $p t=c$.

Similarly $q: N \rightarrow E$ extends uniquely to a (surjective) closed degree zero morphism $\widetilde{q}: G^{+}(N) \rightarrow E$, and we have $\varepsilon \widetilde{q}=\widetilde{\nu}$. Let $\rho: R \rightarrow G^{+}(N)$ be the kernel of $\widetilde{q}$. Then $R \in$ $\operatorname{MF}(X, W)$ (since the kernel of a surjective morphism of vector bundles is a vector bundle). Let $C:=\operatorname{Cone}(\rho)$. As a graded sheaf $C=G^{+}(N) \oplus[1] R$, so $C \in \operatorname{MF}(X, W)$. The natural closed degree zero morphism $(\widetilde{q}, 0): C \rightarrow E$ has cone Cone $((\widetilde{q}, 0))$ in $\operatorname{Acycl}[\operatorname{MF}(X, W)]$, cf. the proof of Lemma 2.7.(a).

The composition $C \stackrel{(\widetilde{q}, 0)}{\longrightarrow} E \stackrel{\varepsilon}{\rightarrow} L$ is a closed degree zero morphism and given by $(\varepsilon \widetilde{q}, 0)=$ $(\widetilde{\nu}, 0)$; its third component is $(c, 0): C=G^{+}(N) \oplus[1] R \rightarrow Q$ and can be factored as $C \stackrel{(t, 0)}{\rightarrow} V \stackrel{p}{\rightarrow} Q$. Hence Lemma 2.18 shows that this composition $C \stackrel{(\widetilde{q}, 0)}{\rightarrow} E \stackrel{\varepsilon}{\rightarrow} L$ is homotopic to zero. So it is zero in the triangulated category $[\operatorname{Coh}(X, W)]$, and the morphism $\varepsilon: E \rightarrow L$ factors there through $\operatorname{Cone}((\widetilde{q}, 0)) \in \operatorname{Acycl}[\operatorname{MF}(X, W)]$. This proves our claim.

Lemma 2.20. The three equivalent conditions (ff1) ${ }^{\mathrm{op}}$, (ff2 $)^{\mathrm{op}}$, (ff3) ${ }^{\mathrm{op}}$ hold for the squares (因4) and (因5).

Proof. The proof of Lemma 2.16 for arbitrary $W: X \rightarrow \mathbb{A}^{1}$ can easily be modified to show this result. Observe that the kernel of a surjective morphism of locally free sheaves (resp. flat quasi-coherent sheaves) is again locally free (resp. flat quasi-coherent). 
The proof of Theorem 2.9 is complete.

We deduce some corollaries from the proof of Theorem 2.9.

Corollary 2.21. We have

$$
\begin{aligned}
{[\operatorname{InjQcoh}(X, W)] \cap \operatorname{Acycl}[\mathrm{Qcoh}(X, W)] } & =0, \\
{[\operatorname{MF}(X, W)] \cap \operatorname{Acycl}[\operatorname{Coh}(X, W)] } & =\operatorname{Acycl}[\operatorname{MF}(X, W)], \\
{[\operatorname{Coh}(X, W)] \cap \operatorname{Acycl}[\mathrm{Qcoh}(X, W)] } & =\operatorname{Acycl}[\operatorname{Coh}(X, W)], \\
{[\operatorname{Locfree}(X, W)] \cap \operatorname{Acycl}[\mathrm{Qcoh}(X, W)] } & =\operatorname{Acycl}[\operatorname{Locfree}(X, W)], \\
{[\operatorname{FlatQ} \operatorname{coh}(X, W)] \cap \operatorname{Acycl}[\mathrm{Qcoh}(X, W)] } & =\operatorname{Acycl}[\operatorname{FlatQcoh}(X, W)] .
\end{aligned}
$$

Proof. The first equality follows immediately from Lemma 2.13. Let $E \in[\operatorname{MF}(X, W)] \cap$ $\operatorname{Acycl}[\operatorname{Coh}(X, W)]$. We have seen in Lemma 2.16 that condition (ff2) ${ }^{\text {op }}$ holds for the square (困1). Applied to $\mathrm{id}_{E}$, this condition shows that $E$ is a direct summand of an object of $\operatorname{Acycl}[\operatorname{MF}(X, W)]$ and hence in $\operatorname{Acycl}[\operatorname{MF}(X, W)]$. This proves the second equality. The remaining equalities are proved similarly using Lemmata 2.15 and 2.20.

Corollary 2.22 (cf. proof of $[\operatorname{Pos} 11 b$, Thm. 3.6]). Let $\operatorname{strict}([\operatorname{InjQ} \operatorname{coh}(X, W)])$ be the strict closure of $[\operatorname{InjQ\operatorname {coh}}(X, W)]$ in $[\mathrm{Q} \operatorname{coh}(X, W)]$. Then

$$
[\mathrm{Q} \operatorname{coh}(X, W)]=\langle\operatorname{strict}([\operatorname{InjQ} \operatorname{coh}(X, W)]), \operatorname{Acycl}[\mathrm{Q} \operatorname{coh}(X, W)]\rangle
$$

is a semi-orthogonal decomposition (see Def. A.10). In particular, Acycl[Qcoh $(X, W)]$ is the left orthogonal of $[\operatorname{InjQ} \operatorname{coh}(X, W)$ in $[\mathrm{Q} \operatorname{coh}(X, W)]$.

Proof. Lemma 2.10.(a) yields for each $F \in[Q \operatorname{coh}(X, W)]$ a triangle $A \rightarrow F \rightarrow J \rightarrow[1] A$ with $A \in \operatorname{Acycl}[\mathrm{Q} \operatorname{coh}(X, W)]$ and $J \in[\operatorname{InjQ} \operatorname{coh}(X, W)]$. Together with Lemma 2.13 this proves the first claim. The second claim follows from Lemma A.11.(b).

Corollary 2.23. The categories $[\mathrm{Q} \operatorname{coh}(X, W)],[\operatorname{InjQcoh}(X, W)], \operatorname{Acycl}[\mathrm{Qcoh}(X, W)]$, and $\mathrm{DQ} \operatorname{coh}(X, W)$ are cocomplete (closed under arbitrary direct sums) and therefore Karoubian, and the functor $[\mathrm{Q} \operatorname{coh}(X, W)] \rightarrow \mathrm{DQ} \operatorname{coh}(X, W)$ preserves direct sums.

Proof. It is clear that $[\mathrm{Q} \operatorname{coh}(X, W)]$ is cocomplete. Note that $[\operatorname{InjQ\operatorname {coh}}(X, W)]$ is cocomplete by Theorem 2.1.(d), and that $\operatorname{Acycl}[\mathrm{Q} \operatorname{coh}(X, W)]$ is cocomplete as the left orthogonal of $[\operatorname{InjQ\operatorname {coh}}(X, W)]$ in $[\mathrm{Q} \operatorname{coh}(X, W)]$, see Lemma 2.22. Now use [BN93, Lemma 1.5 and Prop. 3.2]. Cocompleteness of $\mathrm{DQ} \operatorname{coh}(X, W)$ follows also from Theorem 2.9.(a).

The following definition should be compared with Definition 2.5. Note that $[\operatorname{Sh}(X, W)$ and $[\mathrm{Q} \operatorname{coh}(X, W)]$ are cocomplete.

Definition 2.24. Denote by $\operatorname{Acycl}^{\mathrm{CO}}[\operatorname{Sh}(X, W)]$ the full triangulated subcategory of $\operatorname{Sh}(X, W)$ that contains $\operatorname{Acycl}[\operatorname{Sh}(X, W)]$ and is closed under arbitrary direct sums. Following $[\mathrm{Pos} 11 \mathrm{~b}$, Pos11a] again we define the coderived category $\operatorname{DSh}^{\mathrm{co}}(X, W)$ of $W$-curved dg sheaves as the Verdier quotient

$$
\operatorname{DSh}^{\mathrm{co}}(X, W):=[\operatorname{Sh}(X, W)] / \operatorname{Acycl}^{\mathrm{Co}}[\operatorname{Sh}(X, W)] .
$$

If we define $\mathrm{DQ} \operatorname{coh}^{\mathrm{Co}}(X, W)$ similarly, Corollary 2.23 shows that $\mathrm{DQ} \operatorname{coh}(X, W)=\mathrm{DQ}^{\mathrm{coh}}{ }^{\mathrm{Co}}(X, W)$. 


\section{Theorem 2.25.}

(a) The functor $[\operatorname{InjSh}(X, W)] \rightarrow \operatorname{DSh}^{\mathrm{co}}(X, W)$ is an equivalence.

(b) The functor $\mathrm{DQcoh}(X, W) \rightarrow \mathrm{DSh}^{\mathrm{co}}(X, W)$ is full and faithful.

Proof. (a) implies (b): Note that we have a full and faithful functor $\operatorname{InjQ\operatorname {coh}}(X, W) \rightarrow$ $\operatorname{Inj} \operatorname{Sh}(X, W)$ by Theorem 2.1.(b). Hence $[\operatorname{InjQcoh}(X, W)] \rightarrow[\operatorname{Inj} \operatorname{Sh}(X, W)]$ is full and faithful, and we can use Theorem 2.9.(a).

(a): Adapting the proof of Lemma 2.10.(a) shows: For any $F \in \operatorname{Sh}(X, W)$ there exists an exact sequence $0 \rightarrow F \rightarrow I^{0} \rightarrow I^{1} \rightarrow \ldots$ in $Z_{0}(\operatorname{Sh}(X, W))$ with all $I^{j} \in \operatorname{InjSh}(X, W)$. It follows from Lemma 2.26 below that the obvious morphism $F \rightarrow \operatorname{Tot}(I)$ has cone in $\operatorname{Acycl}^{\mathrm{CO}}[\operatorname{Sh}(X, W)]$ and hence becomes an isomorphism in $\operatorname{DSh}^{\mathrm{Co}}(X, W)$. Theorem 2.1.(d) shows that $\operatorname{Tot}(I) \in \operatorname{InjSh}(X, W)$. This implies that $[\operatorname{InjSh}(X, W)] \rightarrow \operatorname{DSh}^{\mathrm{co}}(X, W)$ is essentially surjective.

Adapting the proof of Lemma 2.13 shows that the left orthogonal of $[\operatorname{Inj} \operatorname{Sh}(X, W)]$ in $[\operatorname{Sh}(X, W)]$ contains $\operatorname{Acycl}[\operatorname{Sh}(X, W)]$ and hence $\operatorname{Acycl}^{\mathrm{Co}}[\operatorname{Sh}(X, W)]$ since any left orthogonal is stable under direct sums. Now use condition (ff2) of Proposition B.2.

Lemma 2.26. If $F$ is a bounded below exact complex in $Z_{0}(\operatorname{Sh}(X, W))$, then $\operatorname{Tot}(F) \in$ $\operatorname{Acycl}^{\mathrm{CO}}[\mathrm{Sh}(X, W)]$.

Proof. We can assume that $F=\left(\ldots \rightarrow 0 \rightarrow F^{0} \rightarrow F^{1} \rightarrow \ldots\right)$. Let $F_{\leq n}$ be the subcomplex that coincides with $F$ in degrees $<n$, is zero in degrees $>n$, and whose degree $n$ component is the kernel of $F^{n} \rightarrow F^{n+1}$. We have monomorphisms $F_{\leq 0} \rightarrow F_{\leq 1} \rightarrow F_{\leq 2} \rightarrow \ldots$ of bounded exact complexes, and $F=\operatorname{colim} F_{\leq n}$. Note that there is a short exact sequence

$$
0 \rightarrow \bigoplus_{n \in \mathbb{N}} F_{\leq n} \rightarrow \bigoplus_{n \in \mathbb{N}} F_{\leq n} \rightarrow F \rightarrow 0
$$

of complexes in $Z_{0}(\mathrm{Sh}(X, W))$. Totalizing yields a short exact sequence

$$
0 \rightarrow \bigoplus_{n \in \mathbb{N}} \operatorname{Tot}\left(F_{\leq n}\right) \rightarrow \bigoplus_{n \in \mathbb{N}} \operatorname{Tot}\left(F_{\leq n}\right) \rightarrow \operatorname{Tot}(F) \rightarrow 0
$$

in $Z_{0}(\operatorname{Sh}(X, W))$. Part (a) of Lemma 2.7 shows that this short exact sequence yields a triangle in $\operatorname{DSh}(X, W)$ and a fortiori in $\operatorname{DSh}^{\mathrm{co}}(X, W)$, and part (b) of the same lemma shows that $\bigoplus_{n \in \mathbb{N}} \operatorname{Tot}\left(F_{\leq n}\right) \in \operatorname{Acycl}^{\mathrm{CO}}[\operatorname{Sh}(X, W)]$. Hence $\operatorname{Tot}(F)$ becomes zero in $\operatorname{DSh}^{\mathrm{co}}(X, W)$. The claim follows.

Remark 2.27. We don't know whether $\operatorname{Sh}(X)$ has finite injective dimension. If this is the case the method used to prove Theorem 2.9.(a) easily implies that $[\operatorname{Inj} \operatorname{Sh}(X, W)] \rightarrow$ $\operatorname{DSh}(X, W)$ is an equivalence; moreover Theorem 2.25. (a) then shows that $\operatorname{DSh}(X, W)=$ $\operatorname{DSh}^{\mathrm{co}}(X, W)$ and $\operatorname{Acycl}[\operatorname{Sh}(X, W)]=\operatorname{Acycl}^{\mathrm{co}}[\operatorname{Sh}(X, W)]$.

2.4. Case of constant $W$. We study the case that $W$ is a constant function; recall that this means that $W(X)$ consists of a single point of $\mathbb{A}^{1}=\operatorname{Spec} k[T]$ which is then necessarily closed. First we note that the case of a constant nonzero $W$ is not interesting.

Lemma 2.28. Assume that the function $W$ is constant but $W \neq 0$. Then $[\operatorname{Sh}(X, W)]=$ 0 . In particular, all the subcategories $[\mathrm{Q} \operatorname{coh}(X, W)], \ldots,[\mathrm{MF}(X, W)]$ and all the quotient categories $\operatorname{DSh}(X, W), \operatorname{DQcoh}(X, W), \ldots, \mathbf{M F}(X, W)$ are zero. 
Proof. The assumption implies that the morphism $k[T] \rightarrow \Gamma\left(X, \mathcal{O}_{X}\right), T \mapsto W$, factors as $k[T] \rightarrow k[T] / \mathfrak{p} \rightarrow \Gamma\left(X, \mathcal{O}_{X}\right)$ where $\mathfrak{p} \subset k[T]$ is a maximal ideal $\neq(T)$. In particular $T$ is invertible in the field $k[T] / \mathfrak{p}$, so $W$ is invertible in $\Gamma\left(X, \mathcal{O}_{X}\right)$.

Hence for any $E \in \operatorname{Sh}(X, W)$ the degree one morphism

$$
h:=\left(W^{-1} e_{0}, 0\right) \in \operatorname{End}_{\operatorname{Sh}(X, W)}(E)_{1}=\operatorname{Hom}_{\operatorname{Sh}(X)}\left(E_{0}, E_{1}\right) \oplus \operatorname{Hom}_{\operatorname{Sh}(X)}\left(E_{1}, E_{0}\right)
$$

satisfies $d h=\operatorname{id}_{E}$, i. e. $E$ is isomorphic to zero in $[\operatorname{Sh}(X, W)]$.

Hence let us study the case $W=0$. Given an object $E \in \mathrm{Q} \operatorname{coh}(X, 0)$ we may consider its cohomology $H(E)$ which is just a graded quasi-coherent sheaf with components $H_{0}(E)$ and $H_{1}(E)$. Let

$$
\operatorname{Ex}[\mathrm{Q} \operatorname{coh}(X, 0)]:=\left\{E \in[\mathrm{Q} \operatorname{coh}(X, 0)] \mid H_{p}(E)=0 \text { for all } p \in \mathbb{Z}_{2}\right\},
$$

and define $\operatorname{Ex}[\operatorname{Coh}(X, 0)], \ldots, \operatorname{Ex}[\operatorname{FlatQ} \operatorname{coh}(X, 0)]$ accordingly. These categories are thick subcategories of $[Q \operatorname{coh}(X, 0)], \ldots,[F l a t Q \operatorname{coh}(X, 0]$, respectively, and we can form the corresponding Verdier quotients. The next proposition shows that this yields alternative definitions of the categories DQcoh $(X, 0), \ldots, \operatorname{DFlatQ} \operatorname{coh}(X, 0)$.

Note that any morphism $f: E \rightarrow F$ in $Z_{0}(Q \operatorname{coh}(X, 0))$ induces a morphism $H(f): H(E) \rightarrow$ $H(F)$ on cohomology objects; it is called a quasi-isomorphism if $H(f)$ is an isomorphism. It is easy to see that $H_{0}:[\mathrm{Q} \operatorname{coh}(X, 0)] \rightarrow \mathrm{Q} \operatorname{coh}(X)$ is a cohomological functor.

Remark 2.29. These definitions clearly also make sense for $[\operatorname{Sh}(X, 0)]$. If we knew that $\mathrm{Sh}(X)$ has finite injective dimension, the obvious modification of the proof of the following proposition would show that $\operatorname{Ex}[\operatorname{Sh}(X, 0)]=\operatorname{Acycl}[\operatorname{Sh}(X, 0)]$.

Proposition 2.30. Let $\mathcal{M}$ be $\mathrm{Q} \operatorname{coh}(X, 0), \operatorname{Coh}(X, 0), \operatorname{MF}(X, 0), \operatorname{InjQ} \operatorname{coh}(X, 0), \operatorname{Locfree}(X, 0)$, or FlatQcoh $(X, 0)$. Then

$$
\operatorname{Ex}[\mathcal{M}]=\operatorname{Acycl}[\mathcal{M}]
$$

and in particular $\mathrm{D} \mathcal{M}=[\mathcal{M}] / \operatorname{Ex}[\mathcal{M}]$ (where $\mathrm{D} \operatorname{MF}(X, 0):=\operatorname{MF}(X, 0)$ ). A morphism $f$ in $Z_{0}(\mathcal{M})$ becomes an isomorphism in $\mathrm{D} \mathcal{M}$ if and only if $H(f)$ is a quasi-isomorphism.

Proof. We first prove that $\operatorname{Ex}[\mathrm{Q} \operatorname{coh}(X, 0)]=\operatorname{Acycl}[\mathrm{Q} \operatorname{coh}(X, 0)]$. A diagram chase (or Lemma 2.46 below) shows that the totalization of any short exact sequence (or any bounded exact complex) has vanishing cohomology. By applying the cohomological functor $H_{0}$, any triangle in $[\mathrm{Q} \operatorname{coh}(X, 0)]$ gives rise to a (6-periodic) long exact cohomology sequence, and obviously any direct summand of an object with vanishing cohomology has vanishing cohomology. This implies that $\operatorname{Ex}[\mathrm{Q} \operatorname{coh}(X, 0)] \supset \operatorname{Acycl}[\mathrm{Qcoh}(X, 0)]$.

Conversely let $E \in \operatorname{Ex}[\mathrm{Q} \operatorname{coh}(X, 0)]$. Let $U:=\operatorname{ker} e_{0}$ and $V:=\operatorname{ker} e_{1}$. Let

$$
\begin{aligned}
& (U \rightarrow I)=\left(U \rightarrow I^{0} \stackrel{d_{I}^{0}}{\longrightarrow} I^{1} \stackrel{d_{I}^{1}}{\longrightarrow} \ldots \stackrel{d^{n-1}}{\longrightarrow} I^{n} \rightarrow 0\right), \\
& (V \rightarrow J)=\left(V \rightarrow J^{0} \stackrel{d_{J}^{0}}{\longrightarrow} J^{1} \stackrel{d_{J}^{1}}{\longrightarrow} \ldots \stackrel{d^{n-1}}{\longrightarrow} J^{n} \rightarrow 0\right),
\end{aligned}
$$

be finite injective resolutions in $\mathrm{Q} \operatorname{coh}(X)$. Note that we have a short exact sequence $U \hookrightarrow$ $E_{0} \rightarrow V$. The injective resolutions of $U$ and $V$ combine to an injective resolution of $E_{0}:$ there 
is a morphism $r:[-1] J \rightarrow I$ of complexes in $\mathrm{Q} \operatorname{coh}(X)$ such that its cone Cone $(r)$ (which equals $I \oplus J$ if we forget the differential) fits into the following commutative diagram

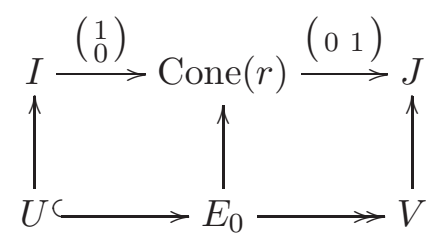

whose columns are injective resolutions. Similarly there is a morphism $s:[-1] I \rightarrow J$ and a commutative diagram

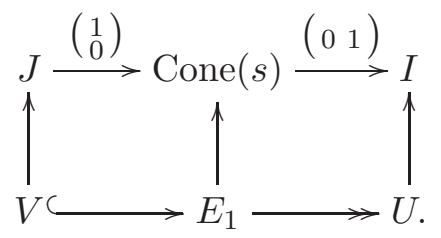

Let $A=\left(A_{1} \underset{a_{0}}{\stackrel{a_{1}}{\rightleftarrows}} A_{0}\right)$ be the complex in $Z_{0}(\mathrm{Q} \operatorname{coh}(X, 0))$ with $A_{0}=\operatorname{Cone}(r), A_{1}=\operatorname{Cone}(s)$ and $a_{0}=\left(\begin{array}{ll}0 & 1 \\ 0 & 0\end{array}\right)$ and $a_{1}=\left(\begin{array}{ll}0 & 1 \\ 0 & 0\end{array}\right)$. Note that we obtain the bounded exact complex

$$
B:=(E \rightarrow A)=\left(\cdots \rightarrow 0 \rightarrow E \rightarrow A^{0} \rightarrow A^{1} \rightarrow \cdots \rightarrow A^{n} \rightarrow 0 \rightarrow \ldots\right)
$$

in $Z_{0}(\mathrm{Qcoh}(X, 0))$. From Lemma 2.7.(c) we obtain a triangle

$$
E \rightarrow \operatorname{Tot}(A) \rightarrow \operatorname{Tot}(B) \rightarrow[1] E
$$

in $[\mathrm{Q} \operatorname{coh}(X, 0)]$. Note that $A^{p}$ is the direct sum of the two objects $J^{p} \underset{1}{\stackrel{0}{\rightleftarrows}} J^{p}$ and $I^{p} \underset{0}{\stackrel{1}{\rightleftarrows}} I^{p}$. Hence Lemma 2.7.(d) implies that $\operatorname{Tot}(A)=0$ in $[\mathrm{Q} \operatorname{coh}(X, 0)]$. Hence $\operatorname{Tot}(B) \stackrel{\sim}{\rightarrow}[1] E$ in $[\mathrm{Q} \operatorname{coh}(X, 0)]$, so $E \in \operatorname{Acycl}[\mathrm{Q} \operatorname{coh}(X, 0)]$ by Lemma 2.7.(b). This proves $\operatorname{Ex}[\mathrm{Q} \operatorname{coh}(X, 0)]=$ $\operatorname{Acycl}[\mathrm{Q} \operatorname{coh}(X, 0)]$.

Now let $\mathcal{M}$ be as in the proposition. Then $\operatorname{Ex}[\mathcal{M}] \supset \operatorname{Acycl}[\mathcal{M}]$ is proved as above, and Corollary 2.21 yields

$$
\operatorname{Ex}[\mathcal{M}] \subset[\mathcal{M}] \cap \operatorname{Ex}[\mathrm{Qcoh}(X, 0)]=[\mathcal{M}] \cap \operatorname{Acycl}[\mathrm{Qcoh}(X, 0)]=\operatorname{Acycl}[\mathcal{M}] .
$$

The last statement is clear: $f$ becomes an isomorphism if and only if its cone is in $\operatorname{Ex}[\mathcal{M}]$; now use the six-periodic long exact sequence obtained from the cohomological functor $H_{0}$.

Remark 2.31. In fact we have proved that each object of $\operatorname{Ex}[\mathrm{Q} \operatorname{coh}(X, 0)]=\operatorname{Acycl}[\mathrm{Qcoh}(X, 0)]$ is isomorphic to the totalization of a bounded exact complex in $Z_{0}(\mathrm{Q} \operatorname{coh}(X, 0)$.

2.5. Derived functors. We recall first some general results about derived functors and then apply them to direct and inverse image functors, and to Hom and tensor functors. 
2.5.1. Reminder on derived functors. We recall results and terminology from the elegant exposition of derived functors in [Mur07] and refer the reader to this note for more details. Let $\mathcal{D}$ be a triangulated category $\mathcal{D}$ with a strict full triangulated subcategory $\mathcal{C}$, and let $F: \mathcal{D} \rightarrow \mathcal{T}$ be a triangulated functor to some other triangulated category $\mathcal{T}$. The question is whether $F$ has a right derived functor $\mathbf{R} F: \mathcal{D} / \mathcal{C} \rightarrow \mathcal{T}$ with respect to $\mathcal{C}$. More precisely, a right derived functor of $F$ with respect to $\mathcal{C}$ is a pair $(\mathbf{R} F, \zeta)$ of a triangulated functor $\mathbf{R} F: \mathcal{D} / \mathcal{C} \rightarrow \mathcal{T}$ and a suitable natural transformation $\zeta$ satisfying some universal property.

Definition 2.32. An object $A \in \mathcal{D}$ is right $F$-acyclic (with respect to $\mathcal{C}$ ) if the following condition holds: given any morphism $s: A \rightarrow D$ with cone in $\mathcal{C}$, there is a morphism $t: D \rightarrow D^{\prime}$ with cone in $\mathcal{C}$ such that $F(t s)$ is an isomorphism.

Note that $F(A)=0$ if $A$ is right $F$-acyclic and in $\mathcal{C}$ (apply the defining property to $A \rightarrow 0)$.

Theorem 2.33 ([Mur07, Thm. 116]). In the above setting we additionally assume that $\mathcal{C} \subset \mathcal{D}$ is a thick subcategory. Suppose that for every $D \in \mathcal{D}$ there exists a morphism $\eta_{D}: D \rightarrow A_{D}$ with cone in $\mathcal{C}$ and $A_{D}$ right $F$-acyclic with respect to $\mathcal{C}$. Then $F$ admits a right derived functor $(\mathbf{R} F, \zeta)$ with respect to $\mathcal{C}$ with the following properties:

(a) For any $D \in \mathcal{D}$ we have $\mathbf{R} F(D)=F\left(A_{D}\right)$ and $\zeta_{D}=F\left(\eta_{D}\right)$.

(b) An object $D \in \mathcal{D}$ is right $F$-acyclic with respect to $\mathcal{C}$ if and only if $\zeta_{D}$ is an isomorphism in $\mathcal{T}$.

We will apply this theorem several times. When we then write $\mathbf{R} F$ later on we implicitly have used some fixed morphisms $\eta_{D}: D \rightarrow A_{D}$ as in the theorem, or we say explicitly which morphism $\eta_{D}$ we use for a particular object $D$. Usually we assume that $\eta_{D}=\operatorname{id}_{D}$ whenever $D$ is right $F$-acyclic.

Remark 2.34. We explain how the functor $\mathbf{R} F$ from Theorem 2.33 is defined on morphisms. Let $\mathcal{A} \subset \mathcal{D}$ be the full subcategory of all right $F$-acyclic objects, and assume that the assumptions of Theorem 2.33 hold. Then in fact $\mathcal{A}$ is a triangulated subcategory, and $F$ vanishes on $\mathcal{A} \cap \mathcal{C}$. We obtain an induced triangulated functor $\bar{F}: \mathcal{A} / \mathcal{A} \cap \mathcal{C} \rightarrow \mathcal{T}$. Moreover, the natural functor $\mathcal{A} / \mathcal{A} \cap \mathcal{C} \rightarrow \mathcal{D} / \mathcal{C}$ is an equivalence, with a quasi-inverse induced by $D \mapsto A_{D}$. Then $\mathbf{R} F$ is just the composition of this quasi-inverse with $\bar{F}$. This determines $\mathbf{R} F$ on morphisms.

Similar results hold for left derived functors.

2.5.2. Direct and inverse image. Let $Y$ be another scheme satisfying condition (srNfKd), and let $\pi: Y \rightarrow X$ be a morphism. We denote the pullback function $\pi^{*}(W)$ on $Y$ again by $W$.

The usual direct image functor $\pi_{*}: \mathrm{Q} \operatorname{coh}(Y) \rightarrow \mathrm{Q} \operatorname{coh}(X)$ induces the dg functor $\pi_{*}: \mathrm{Q} \operatorname{coh}(Y, W) \rightarrow$ Qcoh $(X, W)$ and on homotopy categories the triangulated functor $\pi_{*}$ : $[\mathrm{Q} \operatorname{coh}(Y, W)] \rightarrow$ $[\mathrm{Q} \operatorname{coh}(X, W)]$. Similarly, the usual inverse image functor $\pi^{*}: \mathrm{Q} \operatorname{coh}(X) \rightarrow \mathrm{Q} \operatorname{coh}(Y)$ induces a dg functor $\pi^{*}: \mathrm{Q} \operatorname{coh}(X, W) \rightarrow \mathrm{Q} \operatorname{coh}(Y, W)$ and a triangulated functor $\pi^{*}:[\mathrm{Q} \operatorname{coh}(X, W)] \rightarrow$ 
$[\mathrm{Q} \operatorname{coh}(Y, W)]$. The adjunction $\left(\pi^{*}, \pi_{*}\right)$ in the usual setting induces an adjunction of dg functors,

$$
\operatorname{Hom}_{\mathrm{Q} c o h(Y, W)}\left(\pi^{*}(E), F\right) \stackrel{\sim}{\rightarrow} \operatorname{Hom}_{\mathrm{Q} c o h}(X, W)\left(E, \pi_{*}(F)\right),
$$

and then an adjunction on triangulated functors. We also denote the compositions

$$
[\mathrm{Q} \operatorname{coh}(Y, W)] \stackrel{\pi_{*}}{\longrightarrow}[\mathrm{Q} \operatorname{coh}(X, W)] \rightarrow \mathrm{DQ} \operatorname{coh}(X, W)
$$

and

$$
[\mathrm{Q} \operatorname{coh}(X, W)] \stackrel{\pi^{*}}{\rightarrow}[\mathrm{Q} \operatorname{coh}(Y, W)] \rightarrow \mathrm{DQ} \operatorname{coh}(Y, W)
$$

by $\pi_{*}$ and $\pi^{*}$, respectively.

\section{Theorem 2.35.}

(a) The functor $\pi_{*}:[\mathrm{Q} \operatorname{coh}(Y, W)] \rightarrow \mathrm{DQ} \operatorname{coh}(X, W)$ has a right derived functor $\mathbf{R} \pi_{*}: \mathrm{DQcoh}(Y, W) \rightarrow$ $\operatorname{DQcoh}(X, W)$ with respect to $\operatorname{Acycl}[\mathrm{Qcoh}(Y, W)]$.

(b) The functor $\pi^{*}:[\mathrm{Q} \operatorname{coh}(X, W)] \rightarrow \mathrm{DQ} \operatorname{coh}(Y, W)$ has a left derived functor $\mathbf{L} \pi^{*}: \operatorname{DQcoh}(X, W) \rightarrow$ $\operatorname{DQcoh}(Y, W)$ with respect to $\operatorname{Acycl}[\mathrm{Qcoh}(X, W)]$. This left derived functor maps $\operatorname{DCoh}(X, W)$ to $\operatorname{DCoh}(Y, W)$ and $\mathbf{M F}(X, W)$ to $\mathbf{M F}(Y, W)$. We can assume that $\mathbf{L} \pi^{*}=\pi^{*}: \mathbf{M F}(X, W) \rightarrow \mathbf{M F}(Y, W)$.

(c) There is an adjunction $\left(\mathbf{L} \pi^{*}, \mathbf{R} \pi_{*}\right)$ of triangulated functors.

Proof. (a): Lemma 2.10.(a) provides for each $E \in[\mathrm{Q} \operatorname{coh}(Y, W)]$ a morphism $\eta_{E}: E \rightarrow$ $I_{E}$ with $I_{E} \in[\operatorname{InjQ} \operatorname{coh}(Y, W)]$ and cone in $\operatorname{Acycl}[\mathrm{Qcoh}(Y, W)]$. Hence to apply Theorem 2.33 we need to show that any object $I \in[\operatorname{InjQ\operatorname {coh}}(Y, W)]$ is right $\pi_{*}$-acyclic with respect to $\operatorname{Acycl}[\mathrm{Q} \operatorname{coh}(Y, W)]$. Let $s: I \rightarrow F$ be a morphism in $[\mathrm{Qcoh}(Y, W)]$ with cone in $\operatorname{Acycl}[\mathrm{Q} \operatorname{coh}(Y, W)]$. Apply $\left.\operatorname{Hom}_{[\mathrm{Q} c o h}(Y, W)\right](-, I)$ and use Lemma 2.13. This shows that there is a (unique) morphism $g: F \rightarrow I$ in $[\mathrm{Q} \operatorname{coh}(Y, W)]$ with $g s=\mathrm{id}_{I}$. The octahedral axiom implies that $g$ has cone in $\operatorname{Acycl}[\mathrm{Q} \operatorname{coh}(Y, W)]$, and $g s=\mathrm{id}_{I}$ certainly implies that $\pi_{*}(g s)$ is an isomorphism in $[\mathrm{Q} \operatorname{coh}(X, W)]$ and $\mathrm{DQ} \operatorname{coh}(X, W)$.

(b): Lemma 2.10.(c) yields for each $E \in[Q \operatorname{coh}(X, W)]$ a morphism $\varepsilon_{E}: P_{E} \rightarrow E$ with $P_{E} \in[\operatorname{Locfree}(X, W)] \subset[\operatorname{FlatQ} \operatorname{coh}(X, W)]$ and cone in $\operatorname{Acycl}[\mathrm{Qcoh}(X, W)]$. We want to use the left version of Theorem 2.33. We need to show that any object $P \in[\operatorname{FlatQ} \operatorname{coh}(X, W)]$ is left $\pi^{*}$-acyclic with respect to $\operatorname{Acycl}[\mathrm{Q} \operatorname{coh}(X, W)]$. Let $s: F \rightarrow P$ be a morphism in $[Q \operatorname{coh}(Y, W)]$ with cone in $\operatorname{Acycl}[\mathrm{Q} \operatorname{coh}(Y, W)]$. Consider the morphism $t:=\varepsilon_{F}: P_{F} \rightarrow$ $F$. We need to show that $\pi^{*}(s t)$ is an isomorphism in $\operatorname{DQcoh}(Y, W)$. The cone of $s t$ is in $\operatorname{Acycl}[\mathrm{Qcoh}(X, W)]$, and we can assume that it is in $[\operatorname{FlatQcoh}(X, W)]$. Hence it is enough to show that $\pi^{*}(Q)=0$ in $\operatorname{DQcoh}(Y, W)$ for any $Q \in \operatorname{Acycl}[\operatorname{FlatQcoh}(X, W)]=$ $[$ FlatQcoh $(X, W)] \cap \operatorname{Acycl}[\mathrm{Q} \operatorname{coh}(X, W)]$ (see Corollary 2.21). Certainly we can reduce to the case that $Q=\operatorname{Tot}(G)$, where $G$ is a short exact sequence in $Z_{0}($ FlatQcoh $(X, W))$. But then $\pi^{*}(G)$ is a short exact sequence in $Z_{0}(\mathrm{Q} \operatorname{coh}(Y, W))$, and hence $\pi^{*}(Q)=\operatorname{Tot}\left(\pi^{*}(G)\right)$ is zero in $\mathrm{DQ} \operatorname{coh}(Y, W)$.

Lemma 2.10.(b) shows that we can take $P_{E} \in[\mathrm{MF}(X, W)]$ for $E \in[\operatorname{Coh}(X, W)]$. For $E \in[\mathrm{MF}(X, W)]$ we take $P_{E}=E$.

(c): Apply [Mur07, Thm. 122], whose assumptions are satisfied by the proof of [Mur07, Thm. 116]. 
Remark 2.36. Both $\mathbf{L} \pi^{*}$ and $\mathbf{R} \pi_{*}$ preserve direct sums, cf. Corollary 2.23. This is clear for $\mathbf{L} \pi^{*}$ from the adjunction $\left(\mathbf{L} \pi^{*}, \mathbf{R} \pi_{*}\right)$. For $\mathbf{R} \pi_{*}$ this follows from the above proof: use Corollary 2.23 and the fact that $\pi_{*}$ preserves direct sums since Noetherian schemes are quasi-compact.

Lemma 2.37. Assume that the map $\pi$ is proper. Then the functor $\mathbf{R} \pi_{*}$ maps $\mathbf{M F}(Y, W)$ to (the essential image of) $\mathbf{M F}(X, W)$, and $\left(\pi^{*}, \mathbf{R} \pi_{*}\right)$ is an adjoint pair of functors between the categories $\mathbf{M F}(X, W)$ and $\mathbf{M F}(Y, W)$.

Proof. Let $E \in \mathbf{M F}(Y, W)$. Choose a finite resolution $E \rightarrow I$ as in Lemma 2.10.(a). Then $\mathbf{R} \pi_{*}(E)$ is isomorphic to $\pi_{*}(\operatorname{Tot}(I))=\operatorname{Tot}\left(\pi_{*}(I)\right)$ and the cohomologies of the complex $\pi_{*}(I)$ all lie in $\operatorname{Coh}(X, W)$, by [Gro61, Thm. 3.2.1]. Hence $\mathbf{R} \pi_{*}(E)$ is isomorphic to an object of $\operatorname{DCoh}(X, W)$ by Lemma 2.40.(a) below, and also to an object of $\mathbf{M F}(X, W)$ by Theorem 2.9.(c). This proves the first claim. The second claim is a direct consequence of Theorem 2.35 .

The proof of Theorem 2.35 shows that all objects of $[\operatorname{InjQ\operatorname {coh}}(Y, W)]$ are right $\pi_{*}$-acyclic and that all objects of $[$ FlatQcoh $(X, W)]$ are left $\pi^{*}$-acyclic. Here is an improvement.

Lemma 2.38. Let $E \in[\mathrm{Q} \operatorname{coh}(Y, W)]$ and assume that its components $E_{0}, E_{1}$ are right $\pi_{*}$ acyclic quasi-coherent sheaves in the sense that $R^{i} \pi_{*}\left(E_{p}\right)=0$ for all $p \in \mathbb{Z}_{2}$ and $i \in \mathbb{Z} \backslash\{0\}$. Then $E$ is right $\pi_{*}$-acyclic, so in particular $\pi_{*}(E) \stackrel{\sim}{\rightarrow} \mathbf{R} \pi_{*}(E)$ canonically.

Similarly, if the components of $F \in[\mathrm{Q} \operatorname{coh}(X, W)]$ are left $\pi^{*}$-acyclic quasi-coherent sheaves, then $F$ is left $\pi^{*}$-acyclic, and $\mathbf{L} \pi^{*}(F) \stackrel{\sim}{\rightarrow} \pi^{*}(F)$ canonically.

Proof. Lemma 2.10.(a) provides a finite resolution $E \rightarrow I$ in $Z_{0}(\mathrm{Q} \operatorname{coh}(Y, W))$ with components $I^{l} \in \operatorname{InjQcoh}(Y, W)$. Since all involved quasi-coherent sheaves are $\pi_{*}$-acyclic, $\pi_{*}(E) \rightarrow$ $\pi_{*}(I)$ is still a resolution in $Z_{0}(\mathrm{Q} \operatorname{coh}(X, W))$. Hence the obvious morphism $\pi_{*}(E) \rightarrow$ $\operatorname{Tot}\left(\pi_{*}(I)\right)$ becomes an isomorphism in $\mathrm{DQ} \operatorname{coh}(X, W)$. On the other hand, if we use $E \rightarrow$ $\operatorname{Tot}(I)$ for computing $\mathbf{R} \pi_{*}(E)$, we have $\mathbf{R} \pi_{*}(E)=\pi_{*}(\operatorname{Tot}(I))=\operatorname{Tot}\left(\pi_{*}(I)\right)$ in $\operatorname{DQcoh}(X, W)$. Now Theorem 2.33.(b) shows our first claim. The second claim is proved similarly using Lemma 2.10.(c).

Remark 2.39. If $\pi$ is an affine morphism, all objects of $[\mathrm{Q} \operatorname{coh}(Y, W)]$ are right $\pi_{*}$-acyclic by Lemma 2.38, so $\pi_{*}:[\mathrm{Q} \operatorname{coh}(Y, W)] \rightarrow \mathrm{DQ} \operatorname{coh}(X, W)$ maps $\operatorname{Acycl}[\mathrm{Q} \operatorname{coh}(Y, W)]$ to zero. The induced functor $\pi_{*}: \operatorname{DQ} \operatorname{coh}(Y, W) \rightarrow \mathrm{DQ} \operatorname{coh}(X, W)$ is canonically isomorphic to $\mathbf{R} \pi_{*}$.

If $\pi$ is proper and affine (for example a closed embedding), then all objects of $[\operatorname{Coh}(Y, W)]$ are right acyclic for $\pi_{*}:[\operatorname{Coh}(Y, W)] \rightarrow \operatorname{DCoh}(X, W)$ with respect to $\operatorname{Acycl}[\operatorname{Coh}(Y, W)]$, and hence $\pi_{*}=\mathbf{R} \pi_{*}: \operatorname{DCoh}(Y, W) \rightarrow \operatorname{DCoh}(X, W)$ canonically.

Similarly, if $\pi$ is flat (for example an open embedding), we have $\pi^{*}=\mathbf{L} \pi^{*}$ canonically.

Lemma 2.40. Let $F=\left(0 \rightarrow F^{m} \stackrel{d^{m}}{\longrightarrow} F^{m+1} \rightarrow \ldots \rightarrow F^{n-1} \stackrel{d^{n-1}}{\longrightarrow} F^{n} \rightarrow 0\right)$ be a complex in $Z_{0}(\mathrm{Q} \operatorname{coh}(X, W))$.

(a) Consider the cohomologies $H^{i}(F)$ and the totalization $\operatorname{Tot}(F)$ as objects of $\mathrm{DQ} \operatorname{coh}(X, W)$. Assume that each $H^{i}(F)$ is isomorphic to an object of $\mathbf{M F}(X, W)(r e s p . \operatorname{DCoh}(X, W))$. Then the same is true for $\operatorname{Tot}(F)$. 
(b) Assume that

$$
\operatorname{Hom}_{\mathrm{DQcoh}(X, W)}\left(H^{p}(F),[v] H^{q}(F)\right)=0
$$

for all $p>q$ and $v \in \mathbb{Z}_{2}$ (enough: with $v \equiv q+1-p \bmod 2$ ). Then $\operatorname{Tot}(F) \cong$ $\bigoplus_{i=m}^{n}[i] H^{i}(F)$ in $\operatorname{DQcoh}(X, W)$.

Proof. If $m=n$ all statements are trivial, so assume $m<n$. Consider the (vertical) short exact sequence of complexes

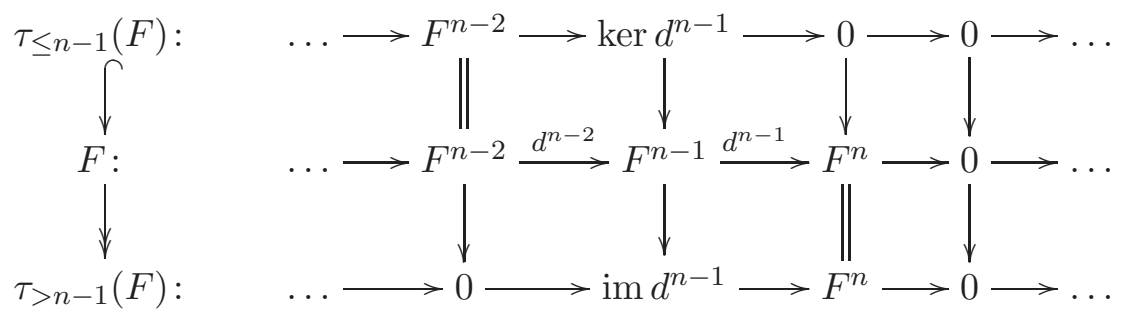

It induces an exact sequence of their totalizations which becomes a triangle in DQcoh $(X, W)$, by Lemma 2.7.(a). The short exact sequence im $d^{n-1} \hookrightarrow F^{n} \rightarrow H^{n}(F)$ gives rise to an isomorphism $\operatorname{Tot}\left(\tau_{>n-1}(F)\right) \stackrel{\sim}{\rightarrow}[n] H^{n}(F)$ in $\operatorname{DQcoh}(X, W)$ by Lemma 2.7.(c). Hence we obtain the triangle

$$
\operatorname{Tot}\left(\tau_{\leq n-1}(F)\right) \rightarrow \operatorname{Tot}(F) \rightarrow[n] H^{n}(F) \rightarrow[1] \operatorname{Tot}\left(\tau_{\leq n-1}(F)\right)
$$

in $\operatorname{DQcoh}(X, W)$.

(a): By induction and our assumptions the first and third object of the triangle (2.1) are isomorphic to objects of $\mathbf{M F}(X, W)$ (resp. $\operatorname{DCoh}(X, W)$ ). The same is then true for $\operatorname{Tot}(F)$.

(b): By induction the triangle (2.1) is isomorphic to the triangle

$$
\bigoplus_{i=m}^{n-1}[i] H^{i}(F) \rightarrow \operatorname{Tot}(F) \rightarrow[n] H^{n}(F) \rightarrow[1] \bigoplus_{i=m}^{n-1}[i] H^{i}(F) .
$$

By assumption the third morphism in this triangle vanishes, and hence $\operatorname{Tot}(F)$ is the direct sum of the first and the third object.

2.5.3. Sheaf Hom and tensor product. Let $W$ and $V$ be arbitrary morphisms $X \rightarrow \mathbb{A}^{1}$. For $P \in \mathrm{Q} \operatorname{coh}(X, W)$ and $Q \in \mathrm{Q} \operatorname{coh}(X, V)$ consider the following diagram ${ }^{2}$

$$
\mathcal{H o m}_{\mathcal{O}_{X}}\left(P_{1}, Q_{0}\right) \oplus \mathcal{H o m}_{\mathcal{O}_{X}}\left(P_{0}, Q_{1}\right) \underset{\left[\begin{array}{cc}
-p_{0}^{*} & q_{1 *} \\
q_{0 *} & -p_{1}^{*}
\end{array}\right]}{\rightleftarrows\left[\begin{array}{cc}
-p_{1}^{*} & q_{1 *} \\
q_{0 *} & -p_{0}^{*}
\end{array}\right]} \mathcal{H o m}_{\mathcal{O}_{X}}\left(P_{0}, Q_{0}\right) \oplus \mathcal{H o m}_{\mathcal{O}_{X}}\left(P_{1}, Q_{1}\right) \text {. }
$$

in the category $\operatorname{Sh}(X)$. It is easy to check that both compositions are multiplication by $V-W$ : note for example that $p_{0}^{*} p_{1}^{*}=-\left(p_{1} p_{0}\right)^{*}$ by the usual sign convention, since $p_{0}$ and $p_{1}$ both have degree one. Hence this diagram defines an object $\mathcal{H o m}(P, Q)$ of $\operatorname{Sh}(X, V-W)$.

\footnotetext{
${ }^{2}$ If $A$ and $B$ are complexes with differentials $d_{A}=a$ and $d_{B}=b$, the differential $d$ in the Hom-complex $\operatorname{Hom}(A, B)$ is given by $d(f)=b \circ f-(-1)^{|f|} f \circ a$ for homogeneous $f$ of degree $|f|$. This explains the signs.
} 
Remark 2.41. In case $W=V$ note that $\mathcal{H o m}(P, Q)$ is in $\operatorname{Sh}(X, 0)$, i. e. it is a dg sheaf, and that

$$
\operatorname{Hom}_{\mathrm{Q} c o h}(X, W)(P, Q)=\Gamma(X, \mathcal{H o m}(P, Q))
$$

as a dg abelian group.

In fact $(P, Q) \mapsto \mathcal{H o m}(P, Q)$ is a dg bifunctor

$$
\mathcal{H o m}(-,-): \mathrm{Q} \operatorname{coh}(X, W)^{\mathrm{op}} \times \mathrm{Q} \operatorname{coh}(X, V) \rightarrow \mathrm{Sh}(X, V-W) .
$$

It induces a bifunctor $\mathcal{H o m}(-,-):[\mathrm{Q} \operatorname{coh}(X, W)]^{\mathrm{op}} \times[\mathrm{Q} \operatorname{coh}(X, V)] \rightarrow[\operatorname{Sh}(X, V-W)]$ of triangulated categories. For fixed $P \in[\mathrm{Q} \operatorname{coh}(X, W)]$ the obvious composition $\mathcal{H o m}(P,-):[\mathrm{Q} \operatorname{coh}(X, V)] \rightarrow$ $\operatorname{DSh}(X, V-W)$ has a right derived functor with respect to $\operatorname{Acycl}[\mathrm{Qcoh}(X, V)]$ : we construct it by choosing morphisms $Q \rightarrow I_{Q}$ with $I_{Q} \in[\operatorname{InjQ} \operatorname{coh}(X, V)]$ and cone in $\operatorname{Acycl}[\mathrm{Q} \operatorname{coh}(X, V)$, for every $Q \in[Q \operatorname{coh}(X, V)]$, and then proceed as in the construction of $\mathbf{R} \pi_{*}$ in the proof of Theorem 2.35 .

For fixed $I \in[\operatorname{InjQ} \operatorname{coh}(X, V)]$, the functor $\mathcal{H o m}(-, I):[\mathrm{Q} \operatorname{coh}(X, W)]^{\text {op }} \rightarrow \operatorname{DSh}(X, V-$ $W)$ maps $\operatorname{Acycl}[\mathrm{Q} \operatorname{coh}(X, W)]$ to zero since $\mathcal{H o m}(-, I)$ maps short exact sequences in $Z_{0}(\mathrm{Q} \operatorname{coh}(X, W))$ to short exact sequences in $Z_{0}(\operatorname{Sh}(X, V-W))$ (use Theorem 2.1.(b)). We define

$$
\begin{aligned}
\mathbf{R} \mathcal{H o m}(-,-): \operatorname{DQ} \operatorname{coh}(X, W)^{\mathrm{op}} \times \operatorname{DQ} \operatorname{coh}(X, V) & \rightarrow \operatorname{DSh}(X, V-W), \\
(P, Q) & \mapsto \mathcal{H o m}\left(P, I_{Q}\right),
\end{aligned}
$$

and leave it to the reader to check that this defines a bifunctor of triangulated categories. Note that for $(P, Q) \in[\mathrm{Q} \operatorname{coh}(X, W)]^{\mathrm{op}} \times[\mathrm{Q} \operatorname{coh}(X, V)]$ there is a natural morphism

$$
\mathcal{H o m}(P, Q) \rightarrow \mathbf{R} \mathcal{H o m}(P, Q)=\mathcal{H o m}\left(P, I_{Q}\right)
$$

in $\operatorname{DSh}(X, V-W)$ induced by $Q \rightarrow I_{Q}$. It is an isomorphism if $P$ is in $[\operatorname{MF}(X, W)]$ (or in $[\operatorname{Locfree}(X, W)])$, or of course if $Q$ is in $[\operatorname{InjQ\operatorname {coh}}(X, V)]$. This also shows that if $P$ is in $\operatorname{Coh}(X, W)$ and we choose $F_{P} \rightarrow P$ in $[\operatorname{Coh}(X, W)]$ with $F_{P} \in[\mathrm{MF}(X, W)]$ and cone in $\operatorname{Acycl}[\mathrm{Q} \operatorname{coh}(X, W)]$, then the morphisms

$$
\mathcal{H o m}\left(F_{P}, Q\right) \rightarrow \mathcal{H o m}\left(F_{P}, I_{Q}\right) \leftarrow \mathcal{H o m}\left(P, I_{Q}\right)
$$

become isomorphisms in $\operatorname{DSh}(X, V-W)$. This gives another way of computing $\mathbf{R} \mathcal{H}$ om $(P, Q)$ for $P \in \operatorname{Coh}(X, W)$.

Note also that $\mathbf{R} \mathcal{H}$ om $(P, Q)$ is in $\operatorname{DQcoh}(X, V-W)$ if $P \in \operatorname{DCoh}(X, W)$, and in (the essential image of $) \operatorname{DCoh}(X, V-W)$ if $P \in \operatorname{DCoh}(X, W)$ and $Q \in \operatorname{DCoh}(X, V)$.

We can also directly obtain a bifunctor

$$
\mathcal{H} \text { om }(-,-): \mathbf{M F}(X, W)^{\mathrm{op}} \times \mathbf{M F}(X, V) \rightarrow \mathbf{M F}(X, V-W)
$$

of triangulated categories. It is isomorphic to the restriction of $\mathbf{R} \mathcal{H o m}(-,-)$ to $\mathbf{M F}(X, W)^{\mathrm{op}} \times$ $\mathbf{M F}(X, V)$. Slightly more general this works also for $\mathcal{H o m}(-,-): \mathbf{M F}(X, W)^{\mathrm{op}} \times \operatorname{DCoh}(X, V) \rightarrow$ $\operatorname{DCoh}(X, V-W)$. 
For $P \in \mathrm{Q} \operatorname{coh}(X, W)$ and $Q \in \mathrm{Q} \operatorname{coh}(X, V)$ note that

$$
\left(P_{1} \otimes_{\mathcal{O}_{X}} Q_{0}\right) \oplus\left(P_{0} \otimes_{\mathcal{O}_{X}} Q_{1}\right) \underset{\left[\begin{array}{l}
\text { id } \otimes q_{1} p_{0} \otimes \text { id } \\
p_{1} \otimes \text { id id } \otimes q_{0}
\end{array}\right]}{\stackrel{\left[\begin{array}{l}
\text { id } \otimes q_{0} \\
p_{1} \otimes \text { id id } \otimes q_{1}
\end{array}\right]}{\rightleftarrows}}\left(P_{1} \otimes_{\mathcal{O}_{X}} Q_{1}\right) \oplus\left(P_{0} \otimes_{\mathcal{O}_{X}} Q_{0}\right)
$$

defines an object $P \otimes Q$ of $\mathrm{Q} \operatorname{coh}(X, V+W)$. We obtain a $\operatorname{dg}$ bifunctor

$$
(-\otimes-): \operatorname{Qcoh}(X, W) \times \mathrm{Q} \operatorname{coh}(X, V) \rightarrow \mathrm{Q} \operatorname{coh}(X, V+W)
$$

and a bifunctor of triangulated categories on homotopy categories. For fixed $P \in[\mathrm{Q} \operatorname{coh}(X, W)]$ the obvious composition $(P \otimes-):[\mathrm{Q} \operatorname{coh}(X, V)] \rightarrow \mathrm{DSh}(X, V+W)$ has a left derived functor with respect to $\operatorname{Acycl}[\mathrm{Q} \operatorname{coh}(X, V)]$ : for each $Q \in[\mathrm{Q} \operatorname{coh}(X, V)]$ we fix a morphism $F_{Q} \rightarrow Q$ with $F_{Q} \in[\operatorname{Locfree}(X, V)] \subset[\operatorname{FlatQ} \operatorname{coh}(X, V)]$ and cone in $\operatorname{Acycl}[\mathrm{Qcoh}(X, V)]$ and proceed then as in the construction of $\mathbf{L} \pi^{*}$ in the proof of Theorem 2.35. It is then easy to see that

$$
\begin{gathered}
\left(-\otimes^{\mathbf{L}}-\right): \operatorname{DQ} \operatorname{coh}(X, W) \times \mathrm{DQ} \operatorname{coh}(X, V) \rightarrow \mathrm{DQ} \operatorname{coh}(X, V+W), \\
(P, Q) \mapsto P \otimes F_{Q},
\end{gathered}
$$

defines a bifunctor of triangulated categories. Again we have for $(P, Q) \in[\mathrm{Q} \operatorname{coh}(X, W)] \times$ $[\mathrm{Q} \operatorname{coh}(X, V)]$ a natural morphism

$$
P \otimes \mathbf{L} Q=P \otimes F_{Q} \rightarrow P \otimes Q
$$

in $\operatorname{DSh}(X, V+W)$ induced by $F_{Q} \rightarrow Q$ which is an isomorphism if $P$ or $Q$ has flat components.

Note that there is an obvious isomorphism

$$
\operatorname{Hom}_{\mathrm{Q} c o h(X, W+V)}(P \otimes Q, R) \stackrel{\sim}{\rightarrow} \operatorname{Hom}_{\mathrm{Q} \operatorname{coh}(X, W)}(P, \mathcal{H o m}(Q, R))
$$

of dg modules which is natural in $P \in \mathrm{Q} \operatorname{coh}(X, W), Q \in \operatorname{Coh}(X, V)$, and $R \in \mathrm{Q} \operatorname{coh}(X, W+$ $V)$.

Lemma 2.42. We have

$$
\operatorname{Hom}_{\mathrm{DQ} \operatorname{coh}(X, W+V)}(P \otimes \mathbf{L} Q, R) \cong \operatorname{Hom}_{\mathrm{DQcoh}(X, W)}(P, \mathbf{R} \mathcal{H} \text { om }(Q, R))
$$

naturally in $P \in \mathrm{DQcoh}(X, W), Q \in \mathrm{DCoh}(X, V)$, and $R \in \mathrm{DQcoh}(X, W+V)$.

Proof. First note that we can assume that $Q \in \mathbf{M F}(X, V)$ and that moreover $R \in \operatorname{InjQ\operatorname {coh}}(X, W+$ $V)$. Then $P \otimes{ }^{\mathbf{L}} Q \stackrel{\sim}{\rightarrow} P \otimes Q$ and $\mathcal{H o m}(Q, R) \stackrel{\sim}{\rightarrow} \mathbf{R} \mathcal{H o m}(Q, R)$. Note also that $\mathcal{H o m}(Q, R) \in$ $\operatorname{InjQ\operatorname {coh}}(X, W)$ by [Har66, Prop. 7.17]. Now take $H_{0}$ of the above isomorphism (2.5) of $\mathrm{dg}$ modules and use Remark 2.14.

2.5.4. External tensor product. Let $Y$ be a scheme such that $Y$ and $X \times Y$ satisfy condition (srNfKd), and let $V: Y \rightarrow \mathbb{A}^{1}$ be a morphism. Let $p: X \times Y \rightarrow X$ and $q: X \times Y \rightarrow Y$ be the projections. We define $W * V:=p^{*}(W)+q^{*}(V)$, so $(W * V)(x, y)=W(x)+V(y)$. We define the dg bifunctor $\square$ by

$$
(-\otimes-):=\left(p^{*}(-) \otimes q^{*}(-)\right): \mathrm{Q} \operatorname{coh}(X, W) \times \mathrm{Q} \operatorname{coh}(Y, V) \rightarrow \mathrm{Q} \operatorname{coh}(X \times Y, W * V) .
$$

This functor immediately induces the bifunctor

$$
(-\otimes-): \operatorname{DQcoh}(X, W) \times \operatorname{DQcoh}(Y, V) \rightarrow \operatorname{DQ} \operatorname{coh}(X \times Y, W * V)
$$


of triangulated categories. This functor coincides with the composition $\left(-\otimes \mathbf{L}_{-}^{\mathbf{L}}\right) \circ\left(\mathbf{L} p^{*} \times \mathbf{L} q^{*}\right)$ (since $p$ and $q$ are flat we have $\mathbf{L} p^{*} \stackrel{\sim}{\rightarrow} p^{*}$ and $\mathbf{L} q^{*} \stackrel{\sim}{\rightarrow} q^{*}$, and moreover $\left(-\otimes^{\mathbf{L}}-\right) \stackrel{\sim}{\rightarrow}(-\otimes-$ ) on objects of the form $\left(p^{*}(P), q^{*}(Q)\right)$, cf. the proof of Lemma 2.38). Note also that $P \otimes Q$ is in $\mathbf{M F}(X \times Y, W * V)$ (resp. $\operatorname{DCoh}(X \times Y, V * W))$ if $P \in \mathbf{M F}(X, W)$ and $Q \in \mathbf{M F}(Y, V)$ (resp. $P \in \operatorname{DCoh}(X, W)$ and $Q \in \operatorname{DCoh}(Y, V)$.

2.5.5. Duality. We introduce a duality $D_{X}$ on the category of matrix factorizations. Let $\mathcal{D}_{X}:=\left(0 \rightleftarrows \mathcal{O}_{X}\right) \in \operatorname{MF}(X, 0)$; note that $\mathcal{O}_{X}$ sits in even degree. Then

$$
D_{X}:=(-)^{\vee}:=\mathcal{H o m}\left(-, \mathcal{D}_{X}\right): \operatorname{MF}(X, W) \rightarrow \operatorname{MF}(X,-W)^{\text {op }}
$$

is a equivalence of dg categories and induces an equivalence

$$
D_{X}:=(-)^{\vee}:=\mathcal{H o m}\left(-, \mathcal{D}_{X}\right): \mathbf{M F}(X, W) \rightarrow \mathbf{M F}(X,-W)^{\mathrm{op}}
$$

of triangulated categories. This is just the functor (2.4) with $\mathcal{D}_{X}$ as its fixed second argument. We refer to $D_{X}$ as the duality since $D_{X}^{2}=$ id naturally. Explicitly, $D_{X}$ maps $P=\left(P_{1} \underset{p_{0}}{\stackrel{p_{1}}{\rightleftarrows}} P_{0}\right)$ to

$$
D_{X}(P)=P^{\vee}=\left(P_{1}^{\vee}=\mathcal{H o m}\left(P_{1}, \mathcal{O}_{X}\right) \underset{p_{0}^{\vee}=-p_{1}^{*}}{\stackrel{p_{1}^{\vee}=-p_{0}^{*}}{\rightleftarrows}} P_{0}^{\vee}=\mathcal{H o m}\left(P_{0}, \mathcal{O}_{X}\right) .\right) .
$$

Occasionally we view the duality as the functor $D_{X}=\mathbf{R} \mathcal{H o m}\left(-, \mathcal{D}_{X}\right): \operatorname{DCoh}(X, W) \rightarrow$ $\mathbf{M F}(X,-W)^{\mathrm{op}}$. The next lemma says that the inverse image functor and duality commute.

Lemma 2.43. Let $\pi: Y \rightarrow X$ be a morphism of schemes satisfying condition (srNfKd), and let $W: X \rightarrow \mathbb{A}^{1}$ be a morphism. Then there is an isomorphism $\pi^{*} \circ D_{X} \stackrel{\sim}{\rightarrow} D_{Y} \circ \pi^{*}$ of functors $\mathbf{M F}(X, W) \stackrel{\sim}{\rightarrow} \mathbf{M F}(Y,-W)^{\mathrm{op}}$.

Proof. For $\mathcal{F} \in \mathrm{Q} \operatorname{coh}(X)$ consider the morphism

$$
\mathcal{H o m}_{\mathcal{O}_{X}}\left(\mathcal{F}, \mathcal{O}_{X}\right) \rightarrow \mathcal{H o m}_{\mathcal{O}_{X}}\left(\mathcal{F}, \pi_{*} \mathcal{O}_{Y}\right)=\pi_{*} \mathcal{H o m}_{\mathcal{O}_{Y}}\left(\pi^{*} \mathcal{F}, \mathcal{O}_{Y}\right) .
$$

The arrow is induced by $\mathcal{O}_{X} \rightarrow \pi_{*} \mathcal{O}_{Y}$, and the equality is the usual adjunction. It corresponds under the adjunction to a morphism $\pi^{*} \mathcal{H o m}_{\mathcal{O}_{X}}\left(\mathcal{F}, \mathcal{O}_{X}\right) \rightarrow \mathcal{H o m}_{\mathcal{O}_{Y}}\left(\pi^{*} \mathcal{F}, \mathcal{O}_{Y}\right)$. This morphism is an isomorphism if $\mathcal{F}$ is a vector bundle. From this we obviously obtain the isomorphism we want.

2.6. Enhancements. In this section we define several enhancements of $\mathbf{M F}(X, W)$ and show that they are equivalent (see e.g. [LO10] for the definitions). Similarly we define two equivalent enhancements of $\mathrm{DQ} \operatorname{coh}(X, W)$.

2.6.1. Enhancements using injective quasi-coherent sheaves. Recall that the obvious functor $[\operatorname{InjQ\operatorname {coh}}(X, W)] \rightarrow \mathrm{DQ} \operatorname{coh}(X, W)$ is an equivalence (Theorem 2.9.(a)), in other words $\operatorname{InjQ\operatorname {coh}}(X, W)$ is an enhancement of the triangulated category $\operatorname{DQcoh}(X, W)$. This enhancement induces an enhancement for the full subcategory $\mathbf{M F}(X, W) \stackrel{\sim}{\rightarrow} \operatorname{DCoh}(X, W) \subset$ $\operatorname{DQ} \operatorname{coh}(X, W)$ (cf. Theorem 2.9). Namely, let $\operatorname{InjQ}_{\operatorname{coh}}(X, W) \subset \operatorname{InjQ} \operatorname{coh}(X, W)$ be the 
full dg subcategory consisting of objects which are isomorphic in DQcoh $(X, W)$ to an object of $\mathbf{M F}(X, W)$. Then

$$
\left[\operatorname{InjQ}_{\operatorname{MF}}(X, W)\right] \simeq \mathbf{M F}(X, W),
$$

so $\operatorname{InjQcoh}_{\mathbf{M F}}(X, W)$ is an enhancement of $\mathbf{M F}(X, W)$.

2.6.2. Enhancements by $d g$ quotients. There is a different enhancement of $\mathbf{M F}(X, W)$. Namely, let $\operatorname{AcyclMF}(X, W) \subset \operatorname{MF}(X, W)$ be the full dg subcategory consisting of objects that belong to $\operatorname{Acycl}[\operatorname{MF}(X, W)]$. Consider the Drinfeld dg quotient category $\operatorname{MF}(X, W) / \operatorname{AcyclMF}(X, W)$ (which is pretriangulated, cf. [LO10, Lemma 1.5]). Then by [Dri04, Thm. 1.6.2, Thm. 3.4] there is an equivalence

$$
\operatorname{MF}(X, W)=[\operatorname{MF}(X, W)] / \operatorname{Acycl}[\operatorname{MF}(X, W)] \stackrel{\sim}{\rightarrow}[\operatorname{MF}(X, W) / \operatorname{AcyclMF}(X, W)],
$$

hence $\operatorname{MF}(X, W) / \operatorname{AcyclMF}(X, W)$ is an enhancement of $\mathbf{M F}(X, W)$. Similarly, by defining $\operatorname{AcyclCoh}(X, W) \subset \operatorname{Coh}(X, W)$ to consist of those objects that belong to $\operatorname{Acycl}[\operatorname{Coh}(X, W)]$, we see that $\operatorname{Coh}(X, W) / \operatorname{AcyclCoh}(X, W)$ is an enhancement of $\operatorname{DCoh}(X, W) \widetilde{\leftarrow} \mathbf{M F}(X, W)$.

The same approach works for the category $\operatorname{DQcoh}(X, W)$ : Let $\operatorname{AcyclQcoh}(X, W) \subset$ $\mathrm{Q} \operatorname{coh}(X, W)$ be the full dg subcategory consisting of objects that belong to $\operatorname{Acycl}[\mathrm{Qcoh}(X, W)]$. Then

$\operatorname{DQcoh}(X, W)=[\mathrm{Q} \operatorname{coh}(X, W)] / \operatorname{Acycl}[\mathrm{Q} \operatorname{coh}(X, W)] \stackrel{\sim}{\rightarrow}[\mathrm{Q} \operatorname{coh}(X, W) / \operatorname{AcyclQcoh}(X, W)]$, i. e. the $\mathrm{dg}$ quotient $\mathrm{Q} \operatorname{coh}(X, W) / \operatorname{AcyclQ} \operatorname{coh}(X, W)$ is an enhancement of $\mathrm{DQcoh}(X, W)$.

The two enhancements of $\mathrm{DQ} \operatorname{coh}(X, W)$ using injectives resp. dg quotients are equivalent, and similarly for the three enhancements of $\mathbf{M F}(X, W)$. Namely we have the following lemma.

\section{Lemma 2.44.}

(a) The $d g$ categories $\operatorname{InjQcoh}(X, W)$ and $\operatorname{Qcoh}(X, W) / \operatorname{AcyclQcoh}(X, W)$ are quasiequivalent.

(b) The dg categories $\operatorname{MF}(X, W) / \operatorname{AcyclMF}(X, W)$ and $\operatorname{Coh}(X, W) / \operatorname{AcyclCoh}(X, W)$ and $\operatorname{InjQcoh}_{\mathrm{MF}}(X, W)$ are quasi-equivalent.

Proof. (a) The Drinfeld dg quotient comes with the canonical quotient dg functor $\mathrm{Q} \operatorname{coh}(X, W) \rightarrow$ $\operatorname{Qcoh}(X, W) / \operatorname{AcyclQcoh}(X, W)$. Restriction to the dg subcategory $\operatorname{InjQ\operatorname {coh}}(X, W)$ yields the desired quasi-equivalence $\alpha: \operatorname{InjQcoh}(X, W) \rightarrow \mathrm{Qcoh}(X, W) / \operatorname{AcyclQcoh}(X, W)$.

(b) Consider the dg functor $\alpha: \operatorname{InjQcoh}_{\mathbf{M F}}(X, W) \rightarrow Q \operatorname{coh}(X, W) / \operatorname{AcyclQcoh}(X, W)$ obtained by restriction and the canonical dg functor $\beta: \operatorname{MF}(X, W) / \operatorname{AcyclMF}(X, W) \rightarrow$ $\operatorname{Qcoh}(X, W) / \operatorname{AcyclQcoh}(X, W)$. The induced homotopy functors $[\alpha]$ and $[\beta]$ are full and faithful and have the same essential image in $[\mathrm{Qcoh}(X, W) / \operatorname{AcyclQcoh}(X, W)]$. Let $\mathcal{A} \subset$ $\mathrm{Qcoh}(X, W) / \operatorname{AcyclQ} \operatorname{coh}(X, W)$ be the full dg subcategory consisting of objects that belong to this essential image. Then the dg functors

$$
\operatorname{InjQcoh}_{\mathbf{M F}}(X, W) \stackrel{\alpha}{\rightarrow} \mathcal{A} \stackrel{\beta}{\leftarrow} \operatorname{MF}(X, W) / \operatorname{AcyclMF}(X, W)
$$

are the desired quasi-equivalences. Similarly we prove that $\operatorname{Coh}(X, W) / \operatorname{AcyclCoh}(X, W)$ and $\operatorname{InjQcoh}_{\mathbf{M F}}(X, W)$ are quasi-equivalent. 
2.6.3. Morphism oriented Čech enhancement. After some preparations we will define an enhancement for $\mathbf{M F}(X, W)$ whose morphism spaces are defined using Čech complexes.

Let $\mathcal{U}=\left(U_{i}\right)_{i \in I}$ be an open covering of $X$ and let $\mathcal{F}$ be a $\mathrm{dg}$ sheaf on $X$, i. e. an object of $\operatorname{Sh}(X, 0)$. We define a $\mathbb{Z}_{2} \times \mathbb{Z}$-graded abelian group $C^{*}\left(\mathcal{U}, \mathcal{F}_{*}\right)$ as follows: Its component of degree $(p, q) \in \mathbb{Z}_{2} \times \mathbb{Z}$ is

$$
C^{q}\left(\mathcal{U}, \mathcal{F}_{p}\right)=\prod_{\left(i_{0}, \ldots, i_{q}\right) \in I^{q+1}} \mathcal{F}_{p}\left(U_{i_{0}} \cap \cdots \cap U_{i_{q}}\right) .
$$

We turn $C^{*}\left(\mathcal{U}, \mathcal{F}_{*}\right)$ into a double complex as follows: its first differential (in the $p$-direction) is induced by that of $\mathcal{F}$ and its second differential is the usual Čech differential. The Cech complex $C(\mathcal{U}, \mathcal{F})$ is the total complex of $C^{*}\left(\mathcal{U}, \mathcal{F}_{*}\right)$ : Its $m$-th component for $m \in \mathbb{Z}_{2}$ is given by

$$
C(\mathcal{U}, \mathcal{F})_{m}=\bigoplus_{p \in \mathbb{Z}_{2}, q \in \mathbb{Z}, p+q=m} C^{q}\left(\mathcal{U}, \mathcal{F}_{p}\right)
$$

There is an obvious map

$$
\Gamma(X, \mathcal{F}) \rightarrow C(\mathcal{U}, \mathcal{F})
$$

of dg abelian groups.

A different perspective on $C(\mathcal{U}, \mathcal{F})$ is as follows. Taking the Čech complex defines a functor from $\operatorname{Sh}(X)$ to the category of complexes of vector spaces over $k$, and hence maps $\mathcal{F} \in \operatorname{Sh}(X, 0)$ to a complex $C^{*}(\mathcal{U}, \mathcal{F})$ in $Z_{0}(\operatorname{Sh}(\operatorname{Spec} k, 0))$. Its totalization is $C(\mathcal{U}, \mathcal{F})$.

Lemma 2.45. The morphism (2.9) is a quasi-isomorphism if $\mathcal{F}$ is componentwise flabby (i.e. $\mathcal{F}_{0}$ and $\mathcal{F}_{1}$ are flabby).

Proof. This follows from [God73, Thm. 5.2.3] and part (a) of the following Lemma 2.46.

Lemma 2.46. Let $f: A \rightarrow B$ be a morphism of $\mathbb{Z}_{2} \times \mathbb{Z}$-graded double complexes $A=$

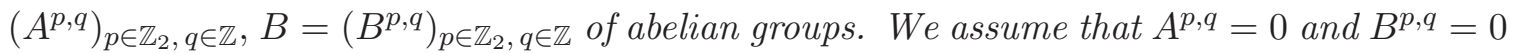
for all $q<M$, for some fixed $M \in \mathbb{Z}$. Assume that one of the following two conditions is true:

(a) f induces isomorphisms $H\left(A^{p, *}\right) \rightarrow H\left(B^{p, *}\right)$ for all $p \in \mathbb{Z}_{2}$.

(b) $f$ induces isomorphisms $H\left(A^{*, q}\right) \rightarrow H\left(B^{*, q}\right)$ for all $q \in \mathbb{Z}$, and $A$ and $B$ are bounded in the $q$-direction, i. e. there is $N \in \mathbb{Z}$ such that $A^{p, q}=0$ and $B^{p, q}=0$ for all $q>N$ and $p \in \mathbb{Z}_{2}$.

Then $f$ induces a quasi-isomorphism $\operatorname{Tot}(f): \operatorname{Tot}(A) \rightarrow \operatorname{Tot}(B)$ of the total complexes associated to $A$ and $B$.

Proof. In this proof we view $A$ and $B$ in the obvious way as $\mathbb{Z} \times \mathbb{Z}$-graded double complexes that are 2-periodic in the $p$-direction.

Assume that (a) holds. Let $F_{n} A$ be the double subcomplex of $A$ defined by

$$
\left(F_{n} A\right)^{p, q}= \begin{cases}A^{p, q} & \text { if } q<n, \\ \operatorname{ker}\left(A^{p, n} \rightarrow A^{p, n+1}\right) & \text { if } q=n, \\ 0 & \text { if } q>n\end{cases}
$$


and similarly for $B$. Then $f$ induces maps $F_{n} f: F_{n} A \rightarrow F_{n} B$ for all $n \in \mathbb{N}$, and these maps induce quasi-isomorphisms on total complexes by [KS94, Thm. 1.9.3]. This obviously implies the claim.

If (b) is satisfied we can immediately apply [KS94, Thm. 1.9.3].

Let us apply the above now to sheaf Hom object $\mathcal{H o m}(E, I)$ defined in section 2.5.3.

Lemma 2.47. Let $E \in \mathrm{Q} \operatorname{coh}(X, W)$ and $I \in \operatorname{InjQ} \operatorname{coh}(X, W)$. Then

$$
\operatorname{Hom}_{\mathrm{Q} c o h(X, W)}(E, I)=\Gamma(X, \mathcal{H o m}(E, I)) \rightarrow C(\mathcal{U}, \mathcal{H o m}(E, I))
$$

(cf. (2.3) and (2.9)) is a quasi-isomorphism. In particular,

$$
\operatorname{Hom}_{\mathrm{DQ} \operatorname{coh}(X, W)}(E,[p] I) \cong H_{p}(C(\mathcal{U}, \mathcal{H} \text { om }(E, I)))
$$

canonically, for $p \in \mathbb{Z}_{2}$.

Proof. Since any injective object of $\mathrm{Q} \operatorname{coh}(X)$ is also an injective object of $\operatorname{Sh}(X)$, by Theorem 2.1.(b), Hom $(E, I)$ is componentwise flabby. Thus Lemma 2.45 shows that the first map is a quasi-isomorphism, and then Remark 2.14 proves the second claim.

Lemma 2.48. Let $\mathcal{F} \rightarrow \mathcal{G}$ be a quasi-isomorphism in $Z_{0}(\mathrm{Q} \operatorname{coh}(X, 0))$. If $\mathcal{U}=\left(U_{i}\right)_{i \in I}$ is an affine open covering of $X$, then $C(\mathcal{U}, \mathcal{F}) \rightarrow C(\mathcal{U}, \mathcal{G})$ is a quasi-isomorphism.

Proof. Since $X$ is quasi-compact there is a finite subset $I^{\prime} \subset I$ such that $\mathcal{U}^{\prime}:=\left(U_{i}\right)_{i \in I^{\prime}}$ is a covering of $X$. If $\mathcal{A}$ is any quasi-coherent sheaf on $X$, the Cech cohomologies $H(\mathcal{U}, \mathcal{A})$ and $H\left(\mathcal{U}^{\prime}, \mathcal{A}\right)$ are canonically isomorphic to $H(X, \mathcal{A})$, since our coverings are by affine open subsets. This together with part (a) of Lemma 2.46 shows that $C\left(\mathcal{U}^{\prime}, \mathcal{F}\right) \rightarrow C(\mathcal{U}, \mathcal{F})$ is an isomorphism.

The usual Čech complex of a sheaf contains the alternating subcomplex and its inclusion is a homotopy equivalence. Similarly, the Čech complex $C\left(\mathcal{U}^{\prime}, \mathcal{F}\right)$ has a homotopy equivalent subcomplex $C_{\text {alt }}\left(\mathcal{U}^{\prime}, \mathcal{F}\right)$.

These arguments show that it is sufficient to show that $C_{\text {alt }}\left(\mathcal{U}^{\prime}, \mathcal{F}\right) \rightarrow C_{\text {alt }}\left(\mathcal{U}^{\prime}, \mathcal{G}\right)$ is a quasi-isomorphism. This follows from part (b) of Lemma 2.46: any finite intersection $U^{\prime}$ of elements of $\mathcal{U}^{\prime}$ is affine, and hence $\mathcal{F}\left(U^{\prime}\right) \rightarrow \mathcal{G}\left(U^{\prime}\right)$ is a quasi-isomorphism by assumption.

Corollary 2.49. Let $E \rightarrow F$ be a morphism in $Z_{0}(\mathrm{Q} \operatorname{coh}(X, W))$ that becomes an isomorphism in $\operatorname{DQcoh}(X, W)$, let $P \in \operatorname{MF}(X, W)$, and let $\mathcal{U}$ be an affine open covering of $X$. Then

$$
C(\mathcal{U}, \mathcal{H o m}(P, E)) \rightarrow C(\mathcal{U}, \mathcal{H o m}(P, F))
$$

is a quasi-isomorphism.

Proof. The morphism $\mathcal{H o m}(P, E) \rightarrow \mathcal{H o m}(P, F)$ in $Z_{0}(\mathrm{Qcoh}(X, 0))$ becomes an isomorphism in $\mathrm{DQcoh}(X, 0)$, cf. section 2.5.3. Hence it is a quasi-isomorphism by Proposition 2.30. Now use Lemma 2.48.

We fix an affine open covering $\mathcal{U}=\left(U_{i}\right)_{i \in I}$ of $X$ for defining the morphism oriented Čech enhancement. We define a dg category $\operatorname{MF}_{\check{C} \text { mor }}(X, W)$ as follows (it depends on the 
affine open covering $\mathcal{U}=\left(U_{i}\right)_{i \in I}$ but we suppress this in the notation). The objects of $\operatorname{MF}_{\text {Ćmor }}(X, W)$ coincide with the objects of $\operatorname{MF}(X, W)$, and the morphisms are given by

$$
\operatorname{Hom}_{\mathrm{MF}_{\check{\mathrm{C}} \text { mor }}(X, W)}(P, Q):=C(\mathcal{U}, \mathcal{H o m}(P, Q)) .
$$

The composition in this category is defined using the cup-product for Čech complexes as defined in [Aut], chapter 18, section 19 "Čech cohomology of complexes" (adapted to our differential $\mathbb{Z}_{2}$-graded situation in the obvious way).

We can repeat this construction starting with any $\mathrm{dg}$ subcategory $\mathcal{C} \subset \operatorname{Qcoh}(X, W)$ to obtain the corresponding $\mathrm{dg}$ category $\mathcal{C}_{\check{C} \text { mor }}$. We always have an obvious $\mathrm{dg}$ functor $\mathcal{C} \rightarrow \mathcal{C}_{\check{C} \text { mor }}$ obtained from $(2.3)$ and $(2.9)$ and the induced functor $[\mathcal{C}] \rightarrow\left[\mathcal{C}_{\breve{C} \text { mor }}\right]$ on homotopy categories.

Proposition 2.50. The $d g$ categories $\operatorname{InjQ}_{\operatorname{coh}}(X, W)$ and $\operatorname{MF}_{\mathrm{C} \text { mor }}(X, W)$ are quasiequivalent, i. e. connected by a zig-zag of quasi-equivalences (explicitly constructed in the proof).

Moreover, $\operatorname{MF}_{\check{C} \text { mor }}(X, W)$ is a pretriangulated dg category, and the functor $[\mathrm{MF}(X, W)] \rightarrow$ $\left[\mathrm{MF}_{\text {C̆mor }}(X, W)\right]$ factors through the Verdier localization $[\mathrm{MF}(X, W)] \rightarrow \mathbf{M F}(X, W)$ to an equivalence

$$
\mathbf{M F}(X, W) \stackrel{\sim}{\rightarrow}\left[\mathrm{MF}_{\text {Čmor }}(X, W)\right]
$$

of triangulated categories. This shows that $\mathrm{MF}_{\check{C} \text { mor }}(X, W)$ is a dg enhancement of $\mathbf{M F}(X, W)$ naturally. We call it the morphism oriented Cech enhancement of $\mathbf{M F}(X, W)$.

In particular this shows that the enhancements $\operatorname{InjQcoh}_{\mathbf{M F}}(X, W)$ and $\operatorname{MF}_{\check{C} \text { mor }}(X, W)$ of $\mathbf{M F}(X, W)$ are equivalent.

Proof. We construct the zig-zag of quasi-equivalences first. To ease the notation we abbreviate $\mathcal{C}:=\operatorname{InjQcoh}_{\mathrm{MF}}(X, W)$. We use the auxiliary dg category $\mathcal{C}_{\text {C̆mor }}$ with the $\operatorname{dg}$ functor $\gamma: \mathcal{C} \rightarrow \mathcal{C}_{\check{C} \text { mor }}$ as explained above. Lemma 2.47 shows that $\gamma$ induces quasi-isomorphisms on morphism spaces. It is bijective on objects and hence a quasi-equivalence.

It remains to prove that the $\mathrm{dg}$ categories $\operatorname{MF}_{\check{C} \text { mor }}(X, W)$ and $\mathcal{C}_{\check{C} \text { mor }}$ are quasi-equivalent. For this we define a new dg category $\mathcal{B}$ and two dg functors

$$
\mathrm{MF}_{\check{\text { C̆mor }}}(X, W) \stackrel{p}{\leftarrow} \mathcal{B} \stackrel{q}{\rightarrow} \mathcal{C}_{\check{C} \text { mor }}
$$

which are quasi-equivalences.

By definition the objects of $\mathcal{B}$ are triples $(P, I, \delta)$, where $P \in \operatorname{MF}(X, W), I \in \mathcal{C}$ and $\delta: P \rightarrow I$ is a morphism in $Z_{0}(\mathrm{Q} \operatorname{coh}(X, W))$ which becomes an isomorphism in $\operatorname{DQcoh}(X, W)$. Given objects $(P, I, \delta)$ and $(Q, J, \varepsilon)$, the $\operatorname{dg}$ module $\operatorname{Hom}_{\mathcal{B}}((P, I, \delta),(Q, J, \varepsilon))$ can be conveniently written in matrix form

$$
\left[\begin{array}{cc}
(I, J) & {[-1](P, J)} \\
0 & (P, Q)
\end{array}\right]
$$

where $(-,-)=\operatorname{Hom}_{\mathrm{Q} \operatorname{coh}(X, W)_{\check{C} \text { mor }}}(-,-)$. The differential is defined by

$$
d:\left[\begin{array}{cc}
r & m \\
0 & l
\end{array}\right] \mapsto\left[\begin{array}{cc}
d r & \varepsilon l-r \delta+d_{[-1](P, J)} m \\
0 & d l
\end{array}\right]=\left[\begin{array}{cc}
d r & \varepsilon l-r \delta-d_{(P, J)} m \\
0 & d l
\end{array}\right],
$$


and composition by

$$
\left[\begin{array}{cc}
\rho & \mu \\
0 & \lambda
\end{array}\right] \circ\left[\begin{array}{cc}
r & m \\
0 & l
\end{array}\right]=\left[\begin{array}{cc}
\rho r & \rho . m+\mu . l \\
0 & \lambda l
\end{array}\right]=\left[\begin{array}{cc}
\rho r & (-1)^{|\rho|} \rho m+\mu l \\
0 & \lambda l
\end{array}\right]
$$

where $m$ is considered as an element of $[-1](P, J)$ in the middle term and as an element of $(P, J)$ in the right term, and similarly for $\mu$.

The obvious projections $\operatorname{MF}_{\text {Čmor }}(X, W) \stackrel{p}{\leftarrow} \mathcal{B} \stackrel{q}{\rightarrow} \mathcal{C}_{\text {C̆mor }}$ are dg functors. These functors are surjective on objects (use Theorem 2.9 and Remark 2.14). Hence in order to show that they are quasi-equivalences we need to see that they induces quasi-isomorphisms on morphism spaces.

Let us prove this for $p$ first. The map $\delta: P \rightarrow I$ yields a closed degree zero morphism $\delta^{*}:(I, J) \rightarrow(P, J)$ in the dg category of dg modules. The shift of its cone Cone $\left(\delta^{*}\right)$ is the kernel of the map $p: \operatorname{Hom}_{\mathcal{B}}((P, I, \delta),(Q, J, \varepsilon)) \rightarrow \operatorname{Hom}_{\mathrm{MF}_{\check{C} \text { mor }}}(P, Q)$. Hence it is sufficient to show that $\operatorname{Cone}\left(\delta^{*}\right)$ is acyclic. Equivalently we show that $\delta^{*}$ is a quasi-isomorphism. But this is true by Lemma 2.47 and Remark 2.14 and our assumption that $\delta$ is an isomorphism in $\operatorname{DQcoh}(X, W)$.

Similarly, when considering $q$, we have to show that $\varepsilon_{*}:(P, Q) \rightarrow(P, J)$ is a quasiisomorphism. But this follows from Corollary 2.49. This shows that $p$ and $q$ are quasiequivalences, and finishes the proof of the first statement.

Our zig-zag of quasi-equivalences yields the equivalences

$$
[\mathcal{C}] \stackrel{[\gamma]}{\longrightarrow}\left[\mathcal{C}_{\check{C} \text { mor }}\right] \stackrel{[q]}{\longleftarrow}[\mathcal{B}] \stackrel{[p]}{\longrightarrow}\left[\mathrm{MF}_{\text {Čmor }}(X, W)\right]
$$

on the level of homotopy categories. This shows that $\operatorname{MF}_{\check{C} \text { mor }}(X, W)$ is pretriangulated. Moreover, if we fix for any $P \in \operatorname{MF}(X, W)$ an object $\left(P, I_{P}, \delta_{P}\right)$ of $\mathcal{B}$, this implies that $P \mapsto I_{P}$ is an equivalence $\left[\mathrm{MF}_{\check{\mathrm{C}} \mathrm{mor}}(X, W)\right] \rightarrow[\mathcal{C}]$.

On the other hand $\mathbf{M F}(X, W) \rightarrow[\mathcal{C}], P \mapsto I_{P}$, is also an equivalence. These two equivalences and the obvious functors fit into the commutative diagram

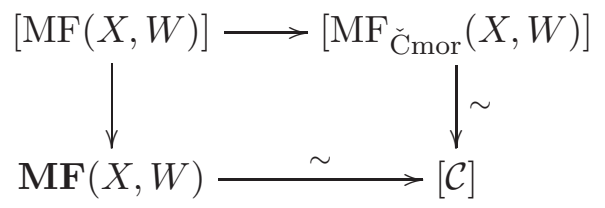

(commutativity is obvious for objects; for morphisms go through the above equivalences) which shows that the upper horizontal functor vanishes on $\operatorname{Acycl}[\operatorname{MF}(X, W)]$. We obtain an induced functor $\mathbf{M F}(X, W) \rightarrow\left[\operatorname{MF}_{\check{C} \text { mor }}(X, W)\right]$ of triangulated categories which is then obviously an equivalence.

Corollary 2.51. The category $\mathrm{MF}_{\text {C̆mor }}(X, W)$ does not depend (up to quasi-equivalence) on the choice of the affine open covering $\mathcal{U}=\left(U_{i}\right)_{i \in I}$ of $X$.

2.6.4. Object oriented Čech enhancement. In [LSb] we will introduce another equivalent enhancement $\operatorname{MF}_{\check{C} \text { obj }}(X, W)$ of $\mathbf{M F}(X, W)$ whose objects are defined using Čech resolutions. 
2.6.5. Enhancement for affine $X$. If $X$ is affine Lemma 2.17.(b) says that $\operatorname{MF}(X, W)$ is an enhancement of $\mathbf{M F}(X, W)$. In fact this enhancement is a special case of the object oriented Čech enhancement (for the trivial affine open covering $\{X\}$ of $X$ ). It is equivalent to the enhancement InjQcoh $\operatorname{MF}_{\mathbf{M F}}(X, W)$ (use the method of proof of Proposition 2.50).

2.7. Compact generators. Recall that the category DQcoh $(X, W)$ is cocomplete (Corollary 2.23).

\section{Proposition 2.52.}

(a) The objects of $\mathbf{M F}(X, W)$ are compact in $\operatorname{DQcoh}(X, W)$.

(b) The triangulated category $\mathrm{DQcoh}(X, W)$ is generated by the objects of $\mathbf{M F}(X, W)$.

(c) The subcategory $\mathrm{DQ} \operatorname{coh}(X, W)^{c}$ of compact objects in $\mathrm{DQ} \operatorname{coh}(X, W)$ is a Karoubi envelope of $\mathbf{M F}(X, W)$. We denote this Karoubi envelope by $\overline{\mathbf{M F}(X, W)}$.

Proof. Results of Neeman [Nee92] imply [BvdB03, Thm. 2.1.2 (and Prop 2.1.1)]. In particular assertions (a) and (b) imply (c).

(a): Follows from Theorem 2.9.(a), Remark 2.14, and Corollary 2.23. Use [Har77, Exercise II.1.11].

(b): We essentially copy the proof of [Pos11b, 3.11 Thm. 2].

Assume that $J \in \operatorname{InjQ\operatorname {coh}}(X, W)$ is such that every morphism $E \rightarrow J$ in $Z_{0}(\operatorname{Qcoh}(X, W))$ with $E \in \operatorname{Coh}(X, W)$ is homotopic to zero. By Theorem 2.9 and Remark 2.14 it suffices to prove that $J=0$ in $[\operatorname{InjQ} \operatorname{coh}(X, W)]$.

Apply Zorn's lemma to the ordered set of pairs $(M, h)$, where $M$ is a subobject of $J$ and $h: M \rightarrow J$ is a contracting homotopy of the embedding $\iota: M \hookrightarrow J$, i. e. $d(h)=\iota$. It suffices to check that given $(M, h)$ with $M \subsetneq J$ there exists $M \subsetneq M^{\prime} \subset J$ and a contracting homotopy $h^{\prime}: M^{\prime} \rightarrow J$ for the embedding $M^{\prime} \hookrightarrow J$ such that $\left.h^{\prime}\right|_{M}=h$. Let $M^{\prime} \subset J$ be a subobject such that $M \subsetneq M^{\prime}$ and $M^{\prime} / M \in \operatorname{Coh}(X, W)$ (use [Har77, Ex. II.5.15.(e)] and the first step in the proof of Lemma 2.15). Since $J$ has injective quasi-coherent components, the degree one morphism $h: M \rightarrow J$ can be extended to a degree one morphism $h^{\prime \prime}: M^{\prime} \rightarrow J$. Let $\iota: M \rightarrow J$ and $\iota^{\prime}: M^{\prime} \rightarrow J$ denote the embeddings. The map $\iota^{\prime}-d\left(h^{\prime \prime}\right)$ is a closed degree zero morphism and vanishes on $M$, so it induces a morphism $g: M^{\prime} / M \rightarrow J$ in $Z_{0}(\mathrm{Q} \operatorname{coh}(X, W))$. By our assumption, there exists a contracting homotopy $c: M^{\prime} / M \rightarrow J$ for $g$. Denote the composition $M^{\prime} \rightarrow M^{\prime} / M \stackrel{c}{\rightarrow} J$ also by $c$. Then $h^{\prime}=h^{\prime \prime}+c: M^{\prime} \rightarrow J$ is a contracting homotopy for $\iota^{\prime}$ extending $h$.

Proposition 2.53. Assume in addition that $X$ is of finite type over $k$. Then the triangulated category $\mathbf{M F}(X, W)$ has a classical generator. Hence so does the category $\overline{\mathbf{M F}(X, W)}$.

Proof. By Remark 2.6 we may assume that $X$ is connected. Then we distinguish two cases: the map $W: X \rightarrow \mathbb{A}^{1}$ is flat or else $W=0$. The remaining case of a constant nonzero $W$ is trivial since then $\mathbf{M F}(X, W)=0$ by Lemma 2.28 .

Assume that $W: X \rightarrow \mathbb{A}^{1}$ is flat. Then by Theorem $2.8 \mathbf{M F}(X, W) \simeq D_{\mathrm{Sg}}\left(X_{0}\right)$. It is well-known that the triangulated category $D^{b}\left(\mathrm{Coh}\left(X_{0}\right)\right)$ has a classical generator (the proof of this fact in [Lun10, 6.3.(a)] also works if $k$ is not perfect). Hence also the quotient category $D_{\mathrm{Sg}}\left(X_{0}\right)=D^{b}\left(\operatorname{Coh}\left(X_{0}\right)\right) / \mathfrak{P e r f}\left(X_{0}\right)$ has a classical generator. 
Assume now that $W=0$. In this case we will use the equivalence $\mathbf{M F}(X, 0) \stackrel{\sim}{\rightarrow} \operatorname{DCoh}(X, 0)$ from Theorem 2.9 and the description $\operatorname{DCoh}(X, 0)=[\operatorname{Coh}(X, 0)] / \operatorname{Ex}[\operatorname{Coh}(X, 0)]$ from Proposition 2.30. Consider the usual bounded derived category $D^{b}(\operatorname{Coh}(X))$ of coherent sheaves on $X$. We have the obvious triangulated folding functor $D^{b}(\operatorname{Coh}(X)) \rightarrow \operatorname{DCoh}(X, 0)$ which takes a $\mathbb{Z}$-graded complex of coherent sheaves to the corresponding $\mathbb{Z}_{2}$-graded one. Since the category $D^{b}(\operatorname{Coh}(X))$ has a classical generator it suffices to show that $\operatorname{DCoh}(X, 0)$ is the triangulated envelope of the collection of objects which are in the image of the folding functor.

For every $E \in \operatorname{Coh}(X, 0)$ we have a short exact sequence

$$
\left(\operatorname{im} e_{0} \underset{0}{\stackrel{0}{\rightleftarrows}} \operatorname{im} e_{1}\right) \hookrightarrow E \rightarrow\left(E_{1} / \operatorname{im} e_{0} \underset{0}{\stackrel{0}{\rightleftarrows}} E_{0} / \operatorname{im} e_{1}\right)
$$

in $Z_{0}(\operatorname{Coh}(X, 0))$ and hence a triangle in $\operatorname{DCoh}(X, 0)$, by Lemma 2.7.(a). But it is obvious that any object in $\operatorname{DCoh}(X, 0)$ with zero differential is in the image of the folding functor.

The folding functor appearing in the above proof will be studied in detail in [Sch].

Remark 2.54. The above proof shows that the folding of a classical generator $G$ of $D^{b}(\operatorname{Coh}(X))$ is a classical generator of $\mathrm{DCoh}(X, 0)$. By replacing $G$ by the direct sum of its cohomologies one can assume that $G \in \operatorname{Coh}(X)$. Then $G$ has a finite resolution by vector bundles, and by replacing $G$ by the direct sum of the involved vector bundles we can assume that $G$ itself is a vector bundle. Then the folding of $G$ has the form $(0 \rightleftarrows G) \in \mathbf{M F}(X, 0)$ and is a classical generator of $\mathbf{M F}(X, 0)$.

\subsection{Some useful results.}

Lemma 2.55. Let $E, F \in \mathrm{Q} \operatorname{coh}(X, W)$ and assume that $\operatorname{Hom}_{D(\mathrm{Q} \operatorname{coh}(X))}\left(E_{p},[i] F_{p^{\prime}}\right)=0$ for all $p, p^{\prime} \in \mathbb{Z}_{2}$ and $i \in \mathbb{Z}$. Then $\operatorname{Hom}_{\mathrm{DQ} \operatorname{coh}(X, W)}(E,[q] F)=0$ for all $q \in \mathbb{Z}_{2}$.

Proof. Let $F \rightarrow I$ be as in Lemma 2.10.(a). Then the isomorphism $F \stackrel{\sim}{\rightarrow} \operatorname{Tot}(I)$ in $\operatorname{DQcoh}(X, W)$ and Remark 2.14 imply that we obtain isomorphisms

$\operatorname{Hom}_{\mathrm{DQcoh}(X, W)}(E,[q] F) \stackrel{\sim}{\rightarrow} \operatorname{Hom}_{\mathrm{DQ} \operatorname{coh}(X, W)}(E,[q] \operatorname{Tot}(I)) \stackrel{\sim}{\leftarrow} H_{q}\left(\operatorname{Hom}_{\mathrm{Q} \operatorname{coh}(X, W)}(E, \operatorname{Tot}(I))\right)$. Hence we need to show that $\mathrm{dg}$ module $\operatorname{Hom}_{\mathrm{Q} \operatorname{coh}(X, W)}(E, \operatorname{Tot}(I))$ is acyclic. This dg module is the totalization of the (finite) complex

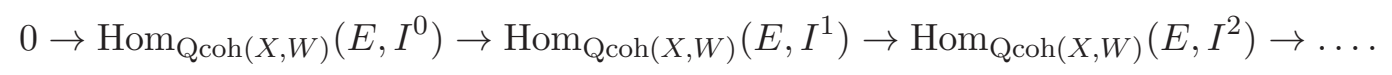

This complex is exact by assumption since $F_{0} \rightarrow I_{0}$ and $F_{1} \rightarrow I_{1}$ are (finite) injective resolutions in the abelian category $\mathrm{Q} \operatorname{coh}(X)$. Hence $\operatorname{Hom}_{\mathrm{Q} c o h(X, W)}(E, \operatorname{Tot}(I))$ is acyclic by Lemma 2.46.(a).

Lemma 2.56 ([Pos11a, Rem. 1.3]). Let $\mathcal{U}$ be an open covering of $X$ and let $E$ be an object of $\mathrm{DQ} \operatorname{coh}(X, W)$. Assume that $\left.E\right|_{U}=0$ in $\mathrm{DQcoh}(X, W)$ for all $U \in \mathcal{U}$. Then $E=0$ in $\operatorname{DQcoh}(X, W)$.

Remark 2.57. The corresponding result for $E \in \mathbf{M F}(X, W)$ can also be shown using Remark 2.6, Lemma 2.28, Proposition 2.30, and Theorem 2.8 (being a perfect complex is defined locally). 
Proof. We repeat the proof of [Pos11a, Rem. 1.3]. We can assume that $\mathcal{U}$ is finite and consists of affine open subsets. For $V \subset X$ open let $j_{V}: V \rightarrow X$ be the inclusion. The Čech resolution

$$
0 \rightarrow E \rightarrow \bigoplus_{U_{0} \in \mathcal{U}} j_{U_{0} *} j_{U_{0}}^{*} E \rightarrow \bigoplus_{U_{0}, U_{1} \in \mathcal{U}} j_{U_{0} \cap U_{1} *} j_{U_{0} \cap U_{1}}^{*} E \rightarrow \ldots
$$

is a bounded exact complex in $Z_{0}(\mathrm{Q} \operatorname{coh}(X, W))$. For any finite intersection $V$ of (a positive number of) elements of $\mathcal{U}$ we have $j_{V}^{*}(E) \in \operatorname{Acycl}[\mathrm{Q} \operatorname{coh}(V, W)]$ by assumption. Since $X$ is separated, $j_{V}$ is affine and hence $j_{V *} j_{V}^{*}(E) \in \operatorname{Acycl}[\mathrm{Qcoh}(X, W)]$ by Remark 2.39. Lemma 2.7.(b)-(d) then shows that $E \in \operatorname{Acycl}[\mathrm{Q} \operatorname{coh}(X, W)]$.

Corollary 2.58. Let $f: E \rightarrow E^{\prime}$ be a morphism in DQcoh $(X, W)$. Assume that $\left.f\right|_{U}:\left.E\right|_{U} \rightarrow$ $\left.E^{\prime}\right|_{U}$ is an isomorphism for all elements $U$ of an open covering of $X$. Then $f$ is an isomorphism.

Proof. A morphism in a triangulated category is an isomorphism if and only if its cone is zero. In our case, this can be checked locally by Lemma 2.56 .

Corollary 2.59. An object $E$ in $[\operatorname{MF}(X, W)]$ belongs to $\operatorname{Acycl}[\operatorname{MF}(X, W)]$ if and only if $E$ is locally contractible, i. e. any point of $X$ has an open neighborhood $U$ such that $E=0$ in $[\mathrm{MF}(U, W)]$.

Proof. If $E$ is locally contractible then $E=0$ in $\operatorname{MF}(X, W)$ by Lemma 2.56, hence $E \in$ $\operatorname{Acycl}[\operatorname{MF}(X, W)]$.

Conversely, let $E \in \operatorname{Acycl}[\operatorname{MF}(X, W)]$. Let $U \subset X$ be any affine open subscheme. Then $\left.E\right|_{U}=0$ in $\operatorname{MF}(U, W)$. But $[\operatorname{MF}(U, W)] \stackrel{\sim}{\rightarrow} \mathbf{M F}(U, W)$ by Lemma 2.17, so $\left.E\right|_{U}$ is contractible.

Proposition 2.60 (Locality of orthogonality). Let $\mathcal{U}$ be an open covering of $X$ and let $A, B \in \mathrm{Q} \operatorname{coh}(X, W)$. Assume that $\operatorname{Hom}_{\mathrm{DQ} \operatorname{coh}\left(U^{\prime}, W\right)}\left(\left.A\right|_{U^{\prime}},\left.[p] B\right|_{U^{\prime}}\right)=0$ for all finite intersections $U^{\prime}$ of elements of $\mathcal{U}$ and all $p \in \mathbb{Z}_{2}$. Then $\operatorname{Hom}_{\mathrm{DQcoh}(X, W)}(A,[p] B)=0$ for all $p \in \mathbb{Z}_{2}$.

Proof. Lemma 2.10.(a) allows us to assume that $B \in \operatorname{InjQcoh}(X, W)$. Then Lemma 2.47 shows that it is enough to prove that $C(\mathcal{U}, \mathcal{H o m}(A, B))$ is acyclic. Since $X$ is quasi-compact we can assume that $\mathcal{U}$ is finite. We order the elements of $\mathcal{U}$, say $\mathcal{U}=\left\{U_{1}, \ldots, U_{n}\right\}$.

As in the proof of Lemma 2.48 it is enough to show that $C_{\text {alt }}(\mathcal{U}, \mathcal{H o m}(A, B))$ is acyclic. Instead of the alternating Cech complex we can work with the isomorphic ordered Čech complex $C_{\text {ord }}(\mathcal{U}, \mathcal{H}$ om $(A, B))$ (defined in the obvious way).

In order to apply Lemma 2.46.(b) it is enough to show the following: for all $q \in \mathbb{N}$ and $1 \leq$ $i_{0}<i_{1}<\cdots<i_{q} \leq n$ the dg module $\mathcal{H o m}(A, B)\left(U^{\prime}\right)$ is acyclic, where $U^{\prime}:=U_{i_{0}} \cap \cdots \cap U_{i_{q}}$. But

$$
\mathcal{H o m}(A, B)\left(U^{\prime}\right)=\Gamma\left(U^{\prime} ; \mathcal{H o m}\left(\left.A\right|_{U^{\prime}},\left.B\right|_{U^{\prime}}\right)\right)=\operatorname{Hom}_{\mathrm{Qcoh}\left(U^{\prime}, W\right)}\left(\left.A\right|_{U^{\prime}},\left.B\right|_{U^{\prime}}\right)
$$

by (2.3), and the latter is acyclic by Theorem 2.1.(c), Remark 2.14, and our assumptions.

Proposition 2.61. Let $X$ and $W: X \rightarrow \mathbb{A}^{1}$ be as before. Let $Z$ be a closed subscheme of $X$ defined by a sheaf of ideals $\mathcal{I} \subset \mathcal{O}_{X}$, and let $U=X-Z$ be its open complement. Let 
$M \in \mathbf{M F}(X, W)$ be such that $\left.M\right|_{U}=0$ in $\mathbf{M F}(U, W)$. Then, for every $n \gg 0$, the canonical morphism $p: M \rightarrow M / \mathcal{I}^{n} M$ has a left inverse $l$ in $\operatorname{DCoh}(X, W)$, i. e. the composition $l \circ p: M \rightarrow M$ is the identity of $M$. In particular, $M$ is isomorphic to a direct summand of $M / \mathcal{I}^{n} M$ in $\operatorname{DCoh}(X, W)$.

Proof. Let $M \rightarrow I$ be a morphism in $Z_{0}(\mathrm{Q} \operatorname{coh}(X, W))$ with $I \in \operatorname{InjQ} \operatorname{coh}(X, W)$ that becomes an isomorphism in DQcoh $(X, W)$ (Lemma 2.10.(a)).

We recall some results from [Har66, II.§7, cf. proof of Thm. 7.18] (see also Theorem 2.1). Any injective quasi-coherent sheaf on $X$ is isomorphic to a direct sum of indecomposable injective quasi-coherent sheaves. Every indecomposable injective quasi-coherent sheaf is isomorphic to some $J(x):=i_{x *}(I(x))$, where $x \in X$ is a point, $i_{x}: \operatorname{Spec} \mathcal{O}_{X, x} \rightarrow X$ is the natural inclusion and $I(x)$ is the injective hull of the $\mathcal{O}_{X, x}$-module $k(x)$.

If a nonzero morphism $J(x) \rightarrow J(y)$ exists, then $y \in \overline{\{x\}}$ : use that $J(x)$ considered as a sheaf of abelian groups is the skyscraper sheaf at $x$ with stalk $I(x)$; this follows from [Har66, Prop. 7.5].

In particular, the components of $I$ are direct sums of indecomposable quasi-coherent sheaves. Denote by $I_{Z} \subset I$ the graded subsheaf consisting of all summands $J(z)$, for $z \in Z$. Then $I_{Z}$ is in fact a subobject, i. e. $I_{Z} \in \operatorname{InjQ\operatorname {coh}}(X, W)$. Let $\varepsilon: U \rightarrow X$ denote the inclusion. The object $\varepsilon^{*} I$ is in $[\operatorname{InjQ} \operatorname{coh}(U, W)]$ by Theorem 2.1.(c), and becomes zero in $\operatorname{DQ} \operatorname{coh}(U, W)$ by assumption. By Theorem 2.9.(a) $\varepsilon^{*} I=0$ in $[\operatorname{InjQ\operatorname {coh}}(U, W)]$, i. e. $\varepsilon^{*} I$ is contractible. Hence the object $\varepsilon_{*} \varepsilon^{*} I \in[\operatorname{InjQ} \operatorname{coh}(X, W)]$ is also contractible. It is easy to check (use that $\varepsilon_{*}$ preserves coproducts) that the sequence

$$
0 \rightarrow I_{Z} \rightarrow I \rightarrow \varepsilon_{*} \varepsilon^{*} I \rightarrow 0
$$

in $Z_{0}(\operatorname{InjQ\operatorname {coh}}(X, W))$ is short exact. Hence $I_{Z} \rightarrow I$ is an isomorphism in $[\operatorname{InjQ\operatorname {coh}}(X, W)]$. Let $I \rightarrow I_{Z}$ in $Z_{0}(\operatorname{InjQ\operatorname {coh}}(X, W))$ represent an inverse. Thus the composition $\alpha: M \rightarrow$ $I \rightarrow I_{Z}$ becomes an isomorphism in DQcoh $(X, W)$. Since the components of $M$ are coherent sheaves and every local section of $I_{Z}$ has support in $Z$, by [Har66, Prop. 7.5], it follows that for some $n_{0} \gg 0$ the morphism $\alpha$ factors as

$$
M \rightarrow M / \mathcal{I}^{n_{0}} M \stackrel{\beta}{\rightarrow} I_{Z}
$$

in $Z_{0}(\mathrm{Q} \operatorname{coh}(X, W))$. But then, in DQcoh $(X, W)$, the composition $\alpha^{-1} \circ \beta: M / \mathcal{I}^{n_{0}} M \rightarrow M$ is the splitting of the projection $M \rightarrow M / \mathcal{I}^{n_{0}}$. Similarly one obtains a splitting of the projection $M \rightarrow M / \mathcal{I}^{n} M$ for any $n>n_{0}$. For the last statement fit $p: M \rightarrow M / \mathcal{I}^{n} M$ into a triangle in $\operatorname{DCoh}(X, W)$ and note that its third morphism is zero.

\section{Semi-orthogonal DeCompositions For matrix FACtorizations ARISING From PROJECTIVE SPACE BUNDLES AND BLOWING-UPS}

There are well-known semi-orthogonal decomposition theorems for bounded derived categories of coherent sheaves on projective space bundles and blowing-ups. We recall them and then state and prove the corresponding results for categories of matrix factorizations. For the definitions of an admissible subcategory and of a semi-orthogonal decomposition we refer to Appendix A. 
3.1. Projective space bundles. Let $Y$ be a scheme satisfying condition (srNfKd), and let $\mathcal{N}$ be a locally free coherent sheaf on $Y$ of rank $r$. Let $E:=\mathbb{P}(\mathcal{N})$ be the associated projective space bundle. It comes with a projection morphism $p: E \rightarrow Y$ and an invertible sheaf $\mathcal{O}(1)=\mathcal{O}_{E}(1)$. Recall the following semi-orthogonal decomposition theorem from ${ }^{3}$ [Or192, BO95], [Huy06, Cor. 8.36].

Theorem 3.1. Assume that $r \geq 1$. Let $l \in \mathbb{Z}$.

$(C o h 1)_{E}$ The functor $\mathcal{O}(l) \otimes p^{*}(-): D^{b}(\operatorname{Coh}(Y)) \rightarrow D^{b}(\operatorname{Coh}(E))$ is full and faithful.

We denote the essential image of this functor by $\mathcal{O}(l) \otimes p^{*} D^{b}(\operatorname{Coh}(Y))$.

$(\text { Coh2 })_{E}$ The subcategory $\mathcal{O}(l) \otimes p^{*} D^{b}(\operatorname{Coh}(Y)) \subset D^{b}(\operatorname{Coh}(E))$ is admissible.

$(C o h 3)_{E}$ The category $D^{b}(\operatorname{Coh}(E))$ has the semi-orthogonal decomposition

$$
D^{b}(\operatorname{Coh}(E))=\left\langle\mathcal{O}(-r+1) \otimes p^{*} D^{b}(\operatorname{Coh}(Y)), \ldots, \mathcal{O}(-1) \otimes p^{*} D^{b}(\operatorname{Coh}(Y)), p^{*} D^{b}(\operatorname{Coh}(Y))\right\rangle .
$$

Now let $W: Y \rightarrow \mathbb{A}^{1}$ be a morphism. We denote the composition $E \stackrel{p}{\rightarrow} Y \stackrel{W}{\rightarrow} \mathbb{A}^{1}$ also by $W$. We have $\mathbf{L} p^{*}=p^{*}: \mathbf{M F}(Y, W) \rightarrow \mathbf{M F}(E, W)$ (see Theorem 2.35.(b)), and tensoring with the line bundle $\mathcal{O}(l)$ induces autoequivalences of the category $\mathbf{M F}(E, W)$. The analog of Theorem 3.1 for matrix factorizations is the following theorem.

Theorem 3.2. Assume that $r \geq 1$. Let $l \in \mathbb{Z}$.

$(M F 1)_{E}$ The functor $\mathcal{O}(l) \otimes p^{*}(-): \mathbf{M F}(Y, W) \rightarrow \mathbf{M F}(E, W)$ is full and faithful.

We denote the essential image of this functor by $\mathcal{O}(l) \otimes p^{*} \mathbf{M F}(Y, W)$.

$(M F 2)_{E}$ The subcategory $\mathcal{O}(l) \otimes p^{*} \mathbf{M F}(Y, W) \subset \mathbf{M F}(E, W)$ is admissible.

$(M F 3)_{E}$ The category $\mathbf{M F}(E, W)$ has the semi-orthogonal decomposition ${ }^{4}$

$$
\mathbf{M F}(E, W)=\left\langle\mathcal{O}(-r+1) \otimes p^{*} \mathbf{M F}(Y, W), \ldots, \mathcal{O}(-1) \otimes p^{*} \mathbf{M F}(Y, W), p^{*} \mathbf{M F}(Y, W)\right\rangle .
$$

Proof of $(M F 1)_{E}$. Note that $\mathcal{O}_{Y} \stackrel{\sim}{\rightarrow} \mathbf{R} p_{*} \mathcal{O}_{E}$. If $V$ is a vector bundle on $Y$, this implies that the adjunction morphism $V \rightarrow \mathbf{R} p_{*} p^{*} V$ is an isomorphism. This means that if $p^{*} V \rightarrow J$ is a (finite) resolution by injective quasi-coherent sheaves, then the obvious morphism $V \rightarrow$ $p_{*}(J)$ is a resolution of $V$. Now let $F \in \mathbf{M F}(Y, W)$ and let $p^{*} F \rightarrow I$ be an exact sequence as in Lemma 2.10.(a). Then the obvious morphism $F \rightarrow p_{*}(I)$ is an exact sequence in $Z_{0}(\mathrm{Q} \operatorname{coh}(Y, W))$, and Lemma 2.7.(c) implies that the adjunction morphism $F \rightarrow \mathbf{R} p_{*} p^{*} F$ is an isomorphism. Hence $p^{*}: \mathbf{M F}(Y, W) \rightarrow \mathbf{M F}(E, W)$ is full and faithful, and this clearly implies $(\mathrm{MF} 1)_{E}$.

Proof of $(M F 2)_{E}$. It is certainly enough to show that $p^{*} \mathbf{M F}(Y, W) \subset \mathbf{M F}(E, W)$ is admissible. By Remark A.5 and its dual version we need to prove that the full and faithful functor $p^{*}: \mathbf{M F}(Y, W) \rightarrow \mathbf{M F}(E, W)$ has a right and a left adjoint. Lemma 2.37 provides a right adjoint $\mathbf{R} p_{*}: \mathbf{M F}(E, W) \rightarrow \mathbf{M F}(Y, W)$. On the other hand, we see from Lemma 2.43 that $D_{Y} \circ \mathbf{R} p_{*} \circ D_{E}$ is left adjoint to $p^{*}$.

\footnotetext{
3 The assumption there is that $Y$ is a smooth projective variety over a field.

4 This is also true for $r=0$ since then $E=\emptyset$.
} 
It remains to prove $(\mathrm{MF} 3)_{E}$. More precisely we need to prove that the specified sequences of admissible subcategories are semi-orthogonal and complete (see Definition A.10).

Proof of semi-orthogonality in $(M F 3)_{E}$. Lemma 2.55 shows that this is a direct consequence of Theorem 3.1.(Coh3) $E$ (and this statement is not difficult to prove using the local-to-global Ext spectral sequence).

We now prepare for the proof of completeness in $(\mathrm{MF} 3)_{E}$.

Recall that the projection $p: E \rightarrow Y$ is a $\mathbb{P}^{r-1}$-bundle. Let $\Omega_{E / Y}$ be the sheaf of relative differentials of $E$ over $Y$ ( $=$ the relative cotangent bundle on $E$ ) and let $\Omega_{E / Y}^{t}=\wedge^{t} \Omega_{E / Y}$ (and $\left.\Omega_{E / Y}^{0}=\mathcal{O}_{E}\right)$. Consider the pullback diagram

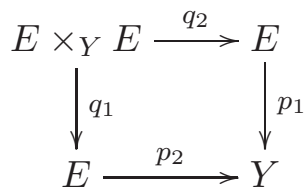

where $p_{1}=p_{2}=p$. We define $F \otimes G:=q_{1}^{*} F \otimes q_{2}^{*} G$ for $F, G \in \operatorname{Coh}(E)$. Denote by $\Delta_{E} \subset E \times_{Y} E$ the diagonal subscheme.

In this situation we have an exact sequence

$$
\begin{aligned}
0 \rightarrow \mathcal{O}_{E}(-r+1) \otimes \Omega_{E / Y}^{r-1}(r-1) & \rightarrow \cdots \rightarrow \mathcal{O}_{E}(-t) \otimes \Omega_{E / Y}^{t}(t) \rightarrow \\
& \cdots \rightarrow \mathcal{O}_{E}(-1) \otimes \Omega_{E / Y}(1) \rightarrow \mathcal{O}_{E \times_{Y} E} \rightarrow \mathcal{O}_{\Delta_{E}} \rightarrow 0
\end{aligned}
$$

in $\operatorname{Coh}\left(E \times_{Y} E\right.$ ) (cf. [Huy06, Remark 8.35]). We denote this locally free resolution of $\mathcal{O}_{\Delta_{E}}$ as $\mathcal{F} \rightarrow \mathcal{O}_{\Delta_{E}}$, i. e. $\mathcal{F}^{-t}=\mathcal{O}_{E}(-t) \otimes \Omega_{E / Y}^{t}(t)$ for $t \geq 0$.

Proof of completeness in $(M F 3)_{E}$. We essentially adapt the proof of [Huy06, Cor. 8.29]. Let $t \geq 0$. For any $T \in \operatorname{Coh}(E)$ we have

$$
\begin{aligned}
\mathbf{R} q_{1 *}\left(\mathcal{F}^{-t}\right. & \left.\otimes q_{2}^{*}(T)\right)=\mathbf{R} q_{1 *}\left(q_{1}^{*}\left(\mathcal{O}_{E}(-t)\right) \otimes q_{2}^{*}\left(\Omega_{E / Y}^{t}(t) \otimes T\right)\right) \\
& =\mathcal{O}_{E}(-t) \otimes \mathbf{R} q_{1 *} q_{2}^{*}\left(\Omega_{E / Y}^{t}(t) \otimes T\right)=\mathcal{O}_{E}(-t) \otimes p_{2}^{*} \mathbf{R} p_{1 *}\left(\Omega_{E / Y}^{t}(t) \otimes T\right)
\end{aligned}
$$

in $D^{b}(\mathrm{Q} \operatorname{coh}(E))$ (or $D^{b}(\operatorname{Coh}(E))$, cf. proof of Lemma 2.37). Indeed the second equality is the projection formula and the third one is flat base change.

In the following we use the exact functor $\operatorname{Coh}\left(E \times_{Y} E\right) \rightarrow Z_{0}\left(\operatorname{Coh}\left(E \times_{Y} E, 0\right)\right), S \mapsto$ $(0 \rightleftarrows S$ ), in order to view coherent sheaves as matrix factorizations. For example, (3.1) can be viewed as a resolution of $\left(0 \rightleftarrows \mathcal{O}_{\Delta_{E}}\right)$ in $Z_{0}\left(\operatorname{Coh}\left(E \times_{Y} E, 0\right)\right)$.

We claim that for $T \in \operatorname{DCoh}(E, W)$ equation (3.2) is also true in $\operatorname{DQcoh}(E, W)$ (or $\operatorname{DCoh}(E, W))$. Just observe that projection formula and flat base change also hold for matrix factorizations. This is easy to prove for the projection formula. For flat base change note that there is a natural morphism and use the following: if $I$ is in $[\operatorname{InjQ} \operatorname{coh}(E, W)]$, then the usual flat base change shows that $q_{2}^{*}(I)$ is right $q_{1 *}$-acyclic, by Lemma 2.38 .

We break the exact sequence (3.1) up into short exact sequences $\mathcal{F}^{-r+1} \hookrightarrow \mathcal{F}^{-r+2} \rightarrow$ $\mathcal{K}^{-r+3}, \ldots, \mathcal{K}^{-t} \hookrightarrow \mathcal{F}^{-t} \rightarrow \mathcal{K}^{-t+1}, \ldots, \mathcal{K}^{0} \hookrightarrow \mathcal{F}^{0} \rightarrow \mathcal{O}_{\Delta_{E}}$. These short exact sequences give rise to triangles in $\operatorname{DCoh}\left(E \times_{Y} E, 0\right)$. 
Let $T \in \operatorname{DCoh}(E, W)$. Form the derived tensor product of $q_{2}^{*}(T)$ with these triangles and apply $\mathbf{R} q_{1 *}$. Using induction and (3.2) we see that

$$
\mathbf{R} q_{1 *}\left(\mathcal{O}_{\Delta_{E}} \otimes^{\mathbf{L}} q_{2}^{*}(T)\right) \in \operatorname{tria}\left(\mathcal{O}_{E}(-r+1) \otimes p_{2}^{*} \operatorname{DCoh}(Y, W), \ldots, p_{2}^{*} \operatorname{DCoh}(Y, W)\right) .
$$

The object on the left is the image of $T$ under the Fourier-Mukai transform with kernel $\mathcal{O}_{\Delta_{E}}$ (in the setting of matrix factorizations). Hence it is isomorphic to $T:$ for $\delta: E \rightarrow E \times_{Y} E$ the (affine) diagonal inclusion we have

$$
\mathcal{O}_{\Delta_{E}} \otimes^{\mathbf{L}} q_{2}^{*}(T)=\delta_{*}\left(\mathcal{O}_{E}\right) \otimes^{\mathbf{L}} q_{2}^{*}(T)=\delta_{*}\left(\mathcal{O}_{E} \otimes^{\mathbf{L}} \delta^{*}\left(q_{2}^{*}(T)\right)\right)=\delta_{*}(T)
$$

by the projection formula, and hence $\mathbf{R} q_{1 *}\left(\mathcal{O}_{\Delta_{E}} \otimes \mathbf{L} q_{2}^{*}(T)\right)=\mathbf{R} q_{1 *}\left(\delta_{*}(T)\right)=T$. Now completeness in $(\mathrm{MF} 3)_{E}$ is immediate from Theorem 2.9.(c).

This finishes the proof of Theorem 3.2

The following result lifts the semi-orthogonal decomposition from Theorem 3.2 to the dg level. We will need it in [LSb]. We use the enhancement by dg quotients explained in section 2.6.2.

Corollary 3.3. There are full $d g$ subcategories $\mathcal{Y}_{l}$ of $\operatorname{MF}(E, W) / \operatorname{AcyclMF}(E, W)$ (for $l \in$ $\mathbb{Z})$ which are quasi-equivalent to $\operatorname{MF}(Y, W) / \operatorname{AcyclMF}(Y, W)$ such that the semi-orthogonal decomposition into admissible subcategories from Theorem 3.2.(MF3) $)_{E}$ is given by

$$
[\operatorname{MF}(E, W) / \operatorname{AcyclMF}(E, W)]=\left\langle\left[\mathcal{Y}_{-r+1}\right], \ldots,\left[\mathcal{Y}_{-1}\right],\left[\mathcal{Y}_{0}\right]\right\rangle
$$

if we identify $\mathbf{M F}(E, W)$ with the left-hand side along (2.8).

Proof. The functor $\mathcal{O}(l) \otimes p^{*}(-): \operatorname{MF}(Y, W) \rightarrow \operatorname{MF}(E, W)$ maps $\operatorname{AcyclMF}(Y, W)$ to $\operatorname{AcyclMF}(E, W)$ and hence induces a dg functor

$$
\mathcal{O}(l) \otimes p^{*}(-): \operatorname{MF}(Y, W) / \operatorname{AcyclMF}(Y, W) \rightarrow \operatorname{MF}(E, W) / \operatorname{AcyclMF}(E, W) .
$$

On homotopy categories this is the full and faithful functor $\mathcal{O}(l) \otimes p^{*}(-): \operatorname{MF}(Y, W) \rightarrow$ $\mathbf{M F}(E, W)$ from $(\mathrm{MF} 1)_{E}$; here and in the following we identify along (2.8). Define $\mathcal{Y}_{l}$ to be the full dg subcategory of $\operatorname{MF}(E, W) / \operatorname{AcyclMF}(E, W)$ consisting of objects that belong to $\mathcal{O}(l) \otimes p^{*} \mathbf{M F}(Y, W)$. All claims follow now from Theorem 3.2.

3.2. Blowing-ups. Now we describe the setting of a blowing-up. Let $X$ be a scheme satisfying condition (srNfKd) and let $i: Y \hookrightarrow X$ be the embedding of a regular equi-codimensional closed subscheme. Let $\pi: \widetilde{X} \rightarrow X$ be the blowing-up of $X$ along $Y$, cf. [Liu02, 8.1] and [GW10, 13], and denote by $j: E \hookrightarrow \widetilde{X}$ the inclusion of the exceptional divisor. We have the following pullback diagram

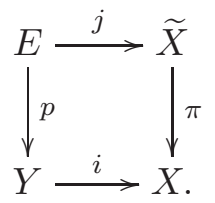

By the usual construction of the blowing-up, $\widetilde{X}$ is endowed with the line bundle $\mathcal{O}(1)=$ $\mathcal{O}_{\widetilde{X}}(1)$. This line bundle is the ideal sheaf corresponding to the closed subscheme $E \subset \widetilde{X}$, i. e. we have a short exact sequence

$$
\mathcal{O}_{\widetilde{X}}(1) \hookrightarrow \mathcal{O}_{\widetilde{X}} \rightarrow \mathcal{O}_{E}
$$


We often denote the restriction $\mathcal{O}_{E}(1)$ of $\mathcal{O}(1)=\mathcal{O}_{\widetilde{X}}(1)$ to $E$ by $\mathcal{O}(1)$ as well.

Let $\mathcal{J} \subset \mathcal{O}_{X}$ be the ideal sheaf of $Y \subset X$. Note that $i$ is a regular immersion of a fixed codimension by [Liu02, 6.3.1]; we denote this codimension by $r$. In particular $\mathcal{J} / \mathcal{J}^{2}$ is locally free of rank $r$ on $Y$. Moreover, the projection $p: E \rightarrow Y$ is a $\mathbb{P}^{r-1}$-bundle (as in subsection 3.1), more precisely it is isomorphic to $\mathbb{P}\left(\mathcal{J} / \mathcal{J}^{2}\right) \rightarrow Y$ (use [Liu02, Thm. 8.1.19], cf. [Har77, Thm. II.8.24]).

Recall the following semi-orthogonal decomposition theorem from ${ }^{5}[\mathrm{Or} 192, \mathrm{BO} 95]^{6}$, [Huy06, Prop. 11.18].

Theorem 3.4. Assume that $r \geq 2$.

$(\text { Coho })_{\tilde{X}}$ The functor $\mathbf{L} \pi^{*}: D^{b}(\operatorname{Coh}(X)) \rightarrow D^{b}(\operatorname{Coh}(\widetilde{X}))$ is full and faithful.

Let $l \in \mathbb{Z}$ and consider the functor

$$
t_{l}(-):=j_{*}\left(\mathcal{O}(l) \otimes p^{*}(-)\right): D^{b}(\operatorname{Coh}(Y)) \rightarrow D^{b}(\operatorname{Coh}(\tilde{X})) .
$$

$(\text { Coh1 })_{\widetilde{X}}$ The functor $t_{l}$ is full and faithful.

Denote by $D^{b}(\operatorname{Coh}(Y))_{l}$ the essential image of $t_{l}$, and by $\mathbf{L} \pi^{*} D^{b}(\operatorname{Coh}(X))$ the essential image of $\mathbf{L} \pi^{*}: D^{b}(\operatorname{Coh}(X)) \rightarrow D^{b}(\operatorname{Coh}(\widetilde{X}))$.

$(\text { Coh2 })_{\tilde{X}}$ The subcategories $D^{b}(\operatorname{Coh}(Y))_{l}$ and $\mathbf{L} \pi^{*} D^{b}(\operatorname{Coh}(X))$ are admissible in $D^{b}(\operatorname{Coh}(\widetilde{X}))$.

$(\text { Coh3 })_{\tilde{X}}$ The category $D^{b}(\operatorname{Coh}(\tilde{X}))$ has the semi-orthogonal decomposition

$$
D^{b}(\operatorname{Coh}(\tilde{X}))=\left\langle D^{b}(\operatorname{Coh}(Y))_{-r+1}, \ldots, D^{b}(\operatorname{Coh}(Y))_{-1}, \mathbf{L} \pi^{*} D^{b}(\operatorname{Coh}(X))\right\rangle .
$$

Now assume that we are given a morphism $W: X \rightarrow \mathbb{A}^{1}$. It induces morphisms from $Y$, $\widetilde{X}$ and $E$ to $\mathbb{A}^{1}$ which we again denote by $W$. Note that $X, Y, \widetilde{X}$, and $E$ satisfy condition ( $\mathrm{srNfKd}$ ). Consider the commutative diagram

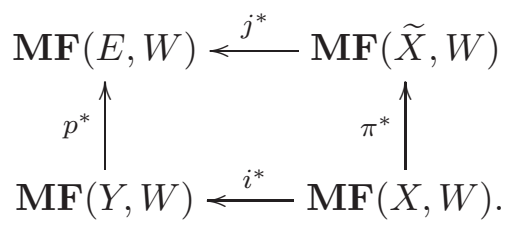

Here $\mathbf{L} \pi^{*}=\pi^{*}$, and similarly for the other functors in this diagram. We also have the functor $j_{*}=\mathbf{R} j_{*}: \mathbf{M F}(E, W) \rightarrow \operatorname{DCoh}(\widetilde{X}, W)$, see Remark 2.39. Note that tensoring with the line bundles $\mathcal{O}(l)$ induces autoequivalences of the categories $\mathbf{M F}(E, W)$ and $\mathbf{M F}(\widetilde{X}, W)$.

Our goal now is to prove the following analog of Theorem 3.4.

Theorem 3.5. Let $r \geq 2$.

$(M F 0)_{\tilde{X}}$ The functor $\pi^{*}: \mathbf{M F}(X, W) \rightarrow \mathbf{M F}(\widetilde{X}, W)$ is full and faithful.

For any integer $l$ consider the functor

$$
s_{l}(-):=j_{*}\left(\mathcal{O}(l) \otimes p^{*}(-)\right): \operatorname{MF}(Y, W) \rightarrow \operatorname{DCoh}(\widetilde{X}, W)
$$

and recall that the latter category is equivalent to $\operatorname{MF}(\widetilde{X}, W)$.

\footnotetext{
5 The assumption there is that $Y$ is a smooth projective variety over a field.

${ }^{6}$ The proof of Theorem 3.4 in [Orl92, BO95] is incomplete. We thank A. Kuznetsov for explaining to us how to fill in the gap. We use his suggestion to prove our Theorem 3.5 below.
} 
$(M F 1)_{\widetilde{X}}$ The functor $s_{l}$ is full and faithful.

Denote by $\pi^{*} \mathbf{M F}(X, W)$ the essential image of $\pi^{*}: \mathbf{M F}(X, W) \rightarrow \mathbf{M F}(\widetilde{X}, W)$, and by

$\mathbf{M F}(Y, W)_{l}$ the intersection of the essential image of $s_{l}$ with $\mathbf{M F}(\widetilde{X}, W)$.

$(M F 2)_{\tilde{X}}$ The subcategory $\pi^{*} \mathbf{M F}(X, W) \subset \mathbf{M F}(\tilde{X}, W)$ is admissible, and so are the subcategories $\mathbf{M F}(Y, W)_{l} \subset \mathbf{M F}(\widetilde{X}, W)$, for any $l \in \mathbb{Z}$.

$(M F 3)_{\tilde{X}}$ The category $\mathbf{M F}(\widetilde{X}, W)$ has the semi-orthogonal decomposition ${ }^{7}$

$$
\mathbf{M F}(\tilde{X}, W)=\left\langle\mathbf{M F}(Y, W)_{-r+1}, \ldots, \mathbf{M F}(Y, W)_{-1}, \pi^{*} \mathbf{M F}(X, W)\right\rangle .
$$

Proof of $(M F 0)_{\tilde{X}}$. We can proceed as in the proof of $(\mathrm{MF} 1)_{E}$ since $\mathcal{O}_{X} \stackrel{\sim}{\rightarrow} \mathbf{R} \pi_{*} \mathbf{L} \pi^{*} \mathcal{O}_{X}=$ $\mathbf{R} \pi_{*} \mathcal{O}_{\widetilde{X}}$ (this follows for example from [SdSSdS09, Thm. 8, Rem. 9]).

Proof of $(M F 1)_{\tilde{X}}$. Fix $\underline{M}, \underline{N} \in \mathbf{M F}(Y, W)$ and $l \in \mathbb{Z}$. Put

$$
M:=\mathcal{O}(l) \otimes p^{*} \underline{M}, \quad N:=\mathcal{O}(l) \otimes p^{*} \underline{N} .
$$

We already know $(\mathrm{MF} 1)_{E}$. Hence it suffices to show that the morphism

$$
j_{*}: \operatorname{Hom}_{\mathbf{M F}(E, W)}(M, N) \rightarrow \operatorname{Hom}_{\mathrm{DCoh}(\widetilde{X}, W)}\left(j_{*} M, j_{*} N\right)
$$

is an isomorphism.

Using the short exact sequence (3.3) and the method used in the proof of Lemma 2.10.(b) we find an exact sequence $0 \rightarrow Q^{-1} \rightarrow Q^{0} \rightarrow j_{*} M \rightarrow 0$ in $Z_{0}(\operatorname{Coh}(\widetilde{X}, W))$ with $Q^{0}$, $Q^{-1} \in \operatorname{MF}(\widetilde{X}, W)$. Let $Q=\left(\cdots \rightarrow 0 \rightarrow Q^{-1} \rightarrow Q^{0} \rightarrow 0 \rightarrow \ldots\right)$, and let $r: \operatorname{Tot}(Q) \rightarrow j_{*} M$ be the obvious morphism. Then by the definition of $\mathbf{L} j^{*}$ we can assume that $\mathbf{L} j^{*} j_{*} M=$ $j^{*}(\operatorname{Tot}(Q))=\operatorname{Tot}\left(j^{*}(Q)\right)$. Consider the composition

$$
\theta: \mathbf{L} j^{*} j_{*}(M)=j^{*}(\operatorname{Tot}(Q)) \stackrel{j^{*}(r)}{\longrightarrow} j^{*} j_{*} M \rightarrow M
$$

where the last morphism is the obvious one. It is enough to show that the morphism

$$
\theta^{*}: \operatorname{Hom}_{\mathbf{M F}(E, W)}(M, N) \rightarrow \operatorname{Hom}_{\mathbf{M F}(E, W)}\left(j^{*}(\operatorname{Tot}(Q)), N\right)
$$

is an isomorphism: if we compose the morphism (3.5) with the isomorphism given by the adjunction in Theorem 2.35, we obtain the morphism (3.4).

Fit $\theta$ into a triangle

$$
C \rightarrow \operatorname{Tot}\left(j^{*}(Q)\right) \stackrel{\theta}{\rightarrow} M \rightarrow[1] C
$$

in $\mathbf{M F}(E, W)$. Applying the cohomological functor $\operatorname{Hom}_{\mathbf{M F}(E, W)}(?, N)$ to this triangle shows that we need to prove that

$$
\operatorname{Hom}_{\mathbf{M F}(E, W)}([v] C, N)=0 \text { for all } v \in \mathbb{Z}_{2} .
$$

By Proposition 2.60 it is sufficient to prove this under the additional assumption that $X$ (and hence $Y$ ) are affine. Moreover we can and will assume that $\underline{M}$ and $\underline{N}$ are free $\mathcal{O}_{Y^{-}}$ modules of finite rank; then $M$ and $N$ are finite direct sums of copies of the line bundle $\mathcal{O}_{E}(l)$.

It is easy to see that $M=H^{0}\left(j^{*}(Q)\right)$ and that $M^{\prime}:=H^{-1}\left(j^{*}(Q)\right)$ coincides with $M(1)$ as a graded vector bundle on $E$ (use the short exact sequence (3.3)). We claim that $C \cong$

\footnotetext{
7 This is also trivially true for $r=0$ and $r=1$.
} 
[1] $M^{\prime}$ in this case, i.e. the morphism $\theta: \operatorname{Tot}\left(j^{*}(Q)\right) \rightarrow M$ fits into a triangle [1] $M^{\prime} \rightarrow$ $\operatorname{Tot}\left(j^{*}(Q)\right) \stackrel{\theta}{\rightarrow} M \rightarrow[2] M^{\prime}$.

Let $A:=j^{*}(Q)$. Let $\tau_{\leq-1}(A)$ be the kernel of the obvious surjective morphism $A \rightarrow$ $H^{0}(A)=M$ of complexes in $Z_{0}(\mathrm{MF}(E, W))$, where $M$ is concentrated in degree 0 . We obtain a short exact sequence $\tau_{\leq-1}(A) \rightarrow A \rightarrow H^{0}(A)=M$ of complexes in $Z_{0}(\mathrm{MF}(E, W))$. Taking totalizations we obtain a short exact sequence in $Z_{0}(\mathrm{MF}(E, W)$ which becomes a triangle in $\mathbf{M F}(E, W)$ (by Lemma 2.7.(a)). On the other hand note that there is an obvious quasi-isomorphism $M^{\prime}=H^{-1}(A) \rightarrow \tau_{\leq-1}(A)$ of complexes in $Z_{0}(\mathrm{MF}(E, W))$, where $M^{\prime}$ is put in degree -1. It gives rise to a morphism [1] $M^{\prime} \rightarrow \operatorname{Tot}\left(\tau_{\leq-1}(A)\right)$ in $Z_{0}(\mathrm{MF}(E, W))$ and to an isomorphism in $\mathbf{M F}(E, W)$. Altogether we obtain the triangle [1] $M^{\prime} \rightarrow \operatorname{Tot}\left(j^{*}(Q)\right) \stackrel{\theta}{\rightarrow}$ $M \rightarrow[2] M^{\prime}$ we claimed to exist, in particular $C \cong[1] M^{\prime}$ in $\mathbf{M F}(E, W)$.

Hence we are reduced to proving that

$$
\operatorname{Hom}_{\mathbf{M F}(E, W)}\left([v] M^{\prime}, N\right)=0 \text { for all } v \in \mathbb{Z}_{2} .
$$

Since $M^{\prime}$ and $M(1)$ coincide (at least) as graded vector bundles this follows from Lemma 2.55 and our assumptions on $\underline{M}$ and $\underline{N}$ since

$$
\operatorname{Hom}_{D(\mathrm{Qcoh}(E))}\left([v] \mathcal{O}_{E}(l+1), \mathcal{O}_{E}(l)\right)=H^{-v}\left(E, \mathcal{O}_{E}(-1)\right)=0
$$

for all $v \in \mathbb{Z}$. Here we use that $p: E \rightarrow Y$ is a $\mathbb{P}^{r-1}$-bundle and that $r-1 \geq 1$. This finishes the proof of $(\mathrm{MF} 1)_{\widetilde{X}}$.

Proposition 3.7 below is essential for the proof of (the second part of) $(\mathrm{MF} 2)_{\tilde{X}}$. It says how $j_{*}$ commutes with the duality (2.6). Its proof will use the following trivial result.

Lemma 3.6. Let $A$ be a ring. Let $p: P \rightarrow M$ be a surjection of $A$-modules and let $q: Q \rightarrow$ $M$ be any morphism of $A$-modules with $Q$ projective. Consider the morphism $(p, q): P \oplus Q \rightarrow$ $M$. Then there is a morphism $l: Q \rightarrow P$ such that the diagram

$$
\begin{gathered}
P \oplus Q \stackrel{(p, 0)}{\longrightarrow} M \\
{\left[\begin{array}{cc}
1 & -l \\
0 & 1
\end{array}\right] \downarrow \sim} \\
P \oplus Q \stackrel{(p, q)}{\longrightarrow} M
\end{gathered}
$$

commutes.

Proof. Since $p$ is surjective and $Q$ is projective there is $l: Q \rightarrow P$ such that $p l=q$.

Proposition 3.7. There is an isomorphism $\tau: j_{*} \circ D_{E} \stackrel{\sim}{\rightarrow}[1](1) D_{\widetilde{X}} \circ j_{*}$ of functors $\operatorname{MF}(E, W) \rightarrow \operatorname{DCoh}(\widetilde{X},-W)^{\mathrm{op}}$.

Proof. We first define the morphism $\tau$ globally and show afterwards locally that it is an isomorphism.

The short exact sequence $(3.3)$ gives rise to a short exact sequence in $Z_{0}(\operatorname{Coh}(\widetilde{X}, 0))$ and then to a triangle

$$
0 \rightleftarrows(1) \mathcal{O}_{\tilde{X}} \hookrightarrow 0 \rightleftarrows \mathcal{O}_{\tilde{X}} \rightarrow 0 \rightleftarrows j_{*} \mathcal{O}_{E} \stackrel{\delta}{\rightarrow}(1) \mathcal{O}_{\tilde{X}} \rightleftarrows 0
$$


in $\operatorname{DCoh}(\widetilde{X}, 0)$. For later use we describe $\delta$ explicitly. Consider the obvious morphisms

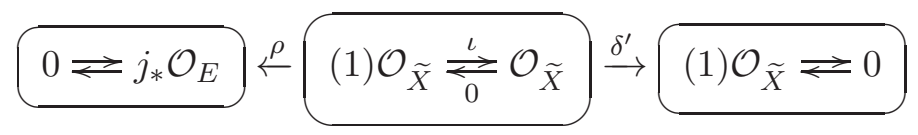

in $Z_{0}(\operatorname{Coh}(\widetilde{X}, 0))$ where the inclusion $(1) \mathcal{O}_{\tilde{X}} \hookrightarrow \mathcal{O}_{\tilde{X}}$ is denoted by $\iota$. The morphism $\rho$ becomes invertible in $\operatorname{DCoh}(\widetilde{X}, 0)$, and there we have $\delta=\delta^{\prime} \circ \rho^{-1}$.

Now define $\tau$ to be the composition

$$
\begin{aligned}
\tau: j_{*} \circ D_{E}=j_{*} \mathbf{R} \mathcal{H o m}_{\mathcal{O}_{E}}\left(-,\left(0 \rightleftarrows \mathcal{O}_{E}\right)\right) \rightarrow \mathbf{R} \mathcal{H o m}_{\mathcal{O}_{\tilde{X}}}\left(j_{*}(-), j_{*}\left(0 \rightleftarrows \mathcal{O}_{E}\right)\right) \\
\stackrel{\delta_{*}}{\longrightarrow} \mathbf{R} \mathcal{H o m}_{\mathcal{O}_{\tilde{X}}}\left(j_{*}(-),\left((1) \mathcal{O}_{\widetilde{X}} \rightleftarrows 0\right)\right)=[1](1) D_{\widetilde{X}} \circ j_{*}
\end{aligned}
$$

where the first morphism is the obvious one and the second one is induced by $\delta$. The last equality is obvious.

Our aim is now to show that $\tau$ is in fact an isomorphism. It is enough to test this locally on an affine open subset $\operatorname{Spec} A \subset \widetilde{X}$ (use Corollary 2.58). We can moreover assume that (3.3) is given by $A \stackrel{f}{\hookrightarrow} A \stackrel{c}{\rightarrow} A / f$, for some $f \in A$.

Let $M=\left(M_{1} \underset{m_{0}}{\stackrel{m_{1}}{\rightleftarrows}} M_{0}\right) \in \mathbf{M F}(\operatorname{Spec} A / f, W)$. By further shrinking Spec $A$ we can and will assume that the components of $M$ are free $A / f$ modules of finite rank, $M_{0}=(A / f)^{\oplus s_{0}}$ and $M_{1}=(A / f)^{\oplus s_{1}}$ for suitable $s_{0}, s_{1} \in \mathbb{N}$.

Let $P_{i}:=A^{\oplus s_{i}}$. We denote the morphisms $c^{\oplus s_{i}}: P_{i} \rightarrow M_{i}$ and $f^{\oplus s_{i}}: P_{i} \hookrightarrow P_{i}$ simply by $c$ and $f$ respectively.

The method of Lemma 2.10.(b) (with a little help from Lemma 3.6) provides the following (vertical) short exact sequence $Q^{-1} \stackrel{q}{\hookrightarrow} Q^{0} \stackrel{r}{\rightarrow} j_{*} M$ in $Z_{0}(\operatorname{Coh}(\operatorname{Spec} A, W)$ ), a two-step resolution of $j_{*}(M)$ by objects of $\operatorname{MF}(\operatorname{Spec} A, W)$.
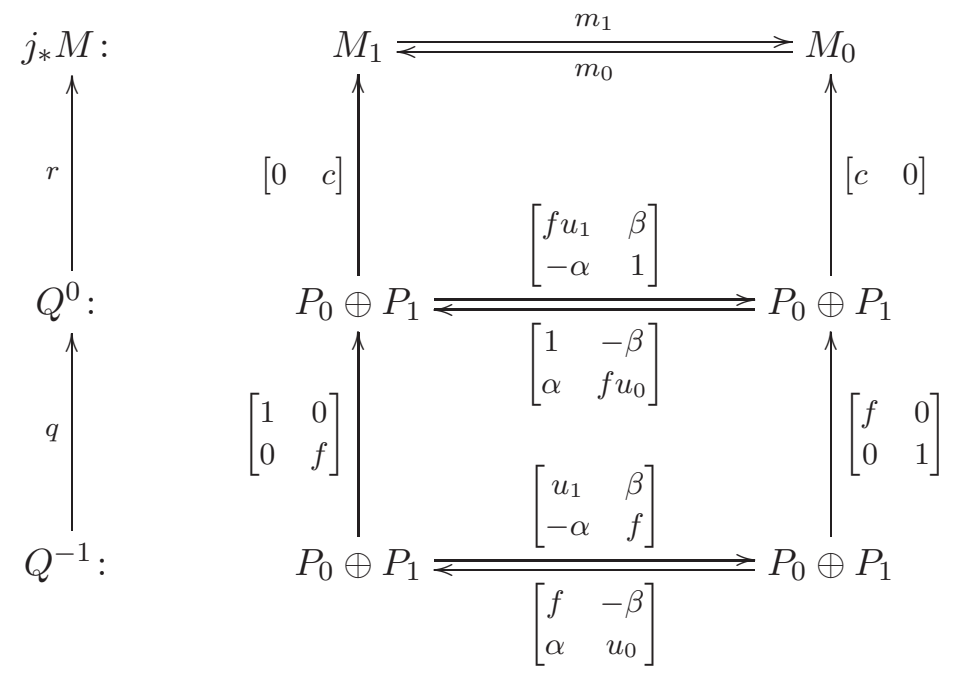

Here $\alpha, \beta, u_{0}, u_{1}$ are suitable morphisms satisfying

$$
\begin{array}{lll}
c \alpha=m_{0} c, & W=f u_{1}+\beta \alpha, & \alpha u_{1}=u_{0} \alpha, \\
c \beta=m_{1} c, & W=f u_{0}+\alpha \beta, & \beta u_{0}=u_{1} \beta .
\end{array}
$$


Note that $Q^{0}$ is isomorphic to zero in $[\operatorname{Coh}(\operatorname{Spec} A, W)]$, as observed in Remark 2.11.

Let $T:=\operatorname{Tot}\left(Q^{-1} \stackrel{q}{\rightarrow} Q^{0}\right)$ be the cone of $q$. Then $r$ defines a morphism $r^{\prime}: T \rightarrow j_{*} M$ in $Z_{0}(\operatorname{Coh}(\operatorname{Spec} A, W))$ that becomes an isomorphism in $\operatorname{DCoh}(\operatorname{Spec} A, W)$. Explicitly $r^{\prime}$ is given by the upper part of the following diagram.

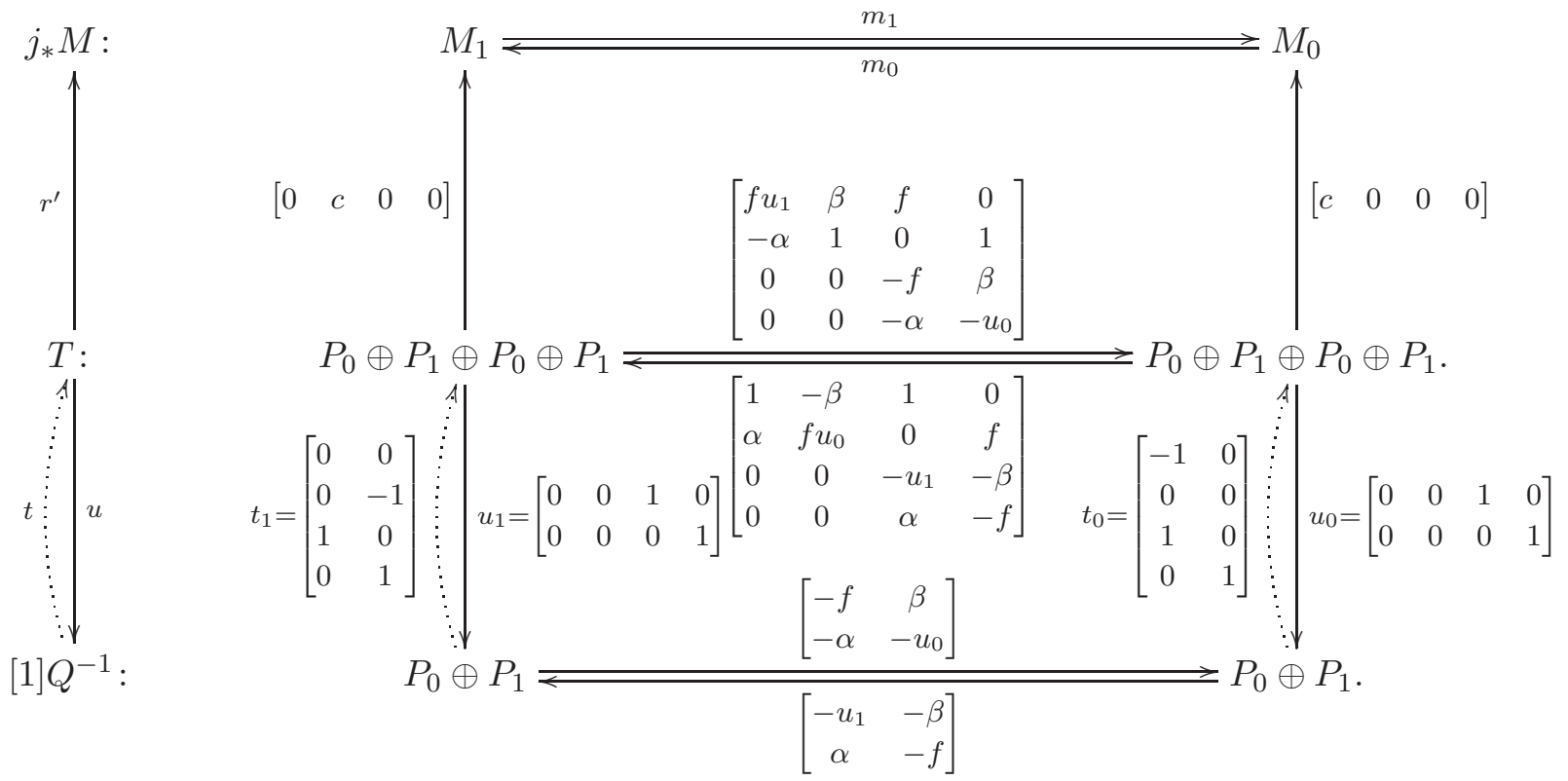

The morphism $u$ in the lower part of this diagram is the obvious projection morphism in the triangle $Q^{-1} \stackrel{q}{\rightarrow} Q^{0} \rightarrow T \stackrel{u}{\rightarrow}[1] Q^{-1}$ in $[\operatorname{Coh}(\operatorname{Spec} A, W)]$. We have observed above that $Q^{0}=0$ in $[\operatorname{Coh}(\operatorname{Spec} A, W)]$, so $u$ is an isomorphism. The dotted morphism $t$ in the diagram in $Z_{0}(\operatorname{Coh}(\operatorname{Spec} A, W))$ represents the inverse of $u$ in $[\operatorname{Coh}(\operatorname{Spec} A, W)]$.

Hence the morphism $r^{\prime \prime}:=r^{\prime} \circ t:[1] Q^{-1} \rightarrow j_{*} M$ in $Z_{0}(\operatorname{Coh}(\operatorname{Spec} A, W))$ becomes an isomorphism in $\operatorname{DCoh}(\operatorname{Spec} A, W)$. It is given by

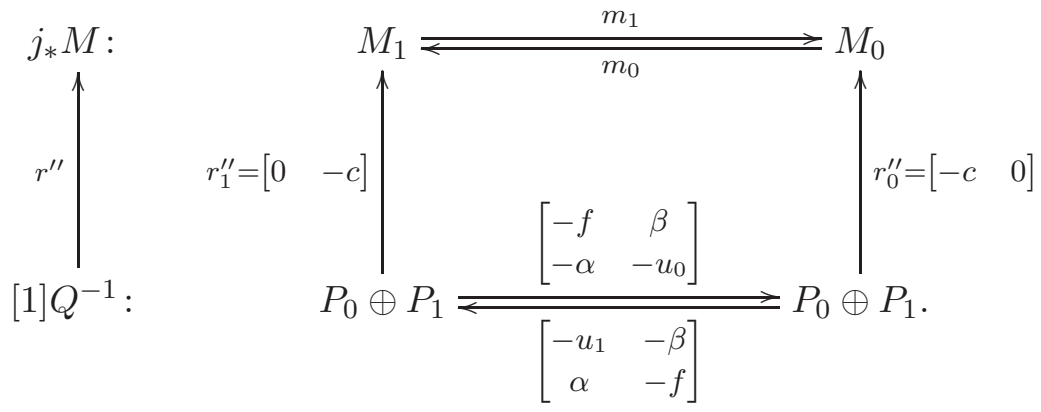

We need to prove that the composition

$$
\begin{aligned}
& \operatorname{Hom}_{A / f}(M,(0 \rightleftarrows A / f)) \\
& \rightarrow \mathcal{E}:=\operatorname{Hom}_{A}\left(j_{*} M,(0 \rightleftarrows A / f)\right) \stackrel{r^{\prime \prime *}}{\longrightarrow} \mathcal{F}:=\operatorname{Hom}_{A}\left([1] Q^{-1},(0 \rightleftarrows A / f)\right) \\
& \quad \stackrel{\left(\rho_{*}\right)^{-1}}{\longrightarrow} \mathcal{G}:=\operatorname{Hom}_{A}\left([1] Q^{-1},(A \underset{0}{\stackrel{f}{\rightleftarrows}} A)\right) \stackrel{\delta_{*}^{\prime}}{\longrightarrow} \mathcal{H}:=\operatorname{Hom}_{A}\left([1] Q^{-1},(A \rightleftarrows 0)\right)
\end{aligned}
$$


in $\operatorname{DCoh}(\operatorname{Spec} A,-W)$ is an isomorphism. The first arrow clearly is an isomorphism. The first (resp. last) two arrows correspond to the first (resp. second) arrow in the definition (3.6) of $\tau_{M}$. The following diagram in $Z_{0}(\operatorname{Coh}(\operatorname{Spec} A,-W)) \operatorname{depicts} \mathcal{E} \stackrel{r^{\prime \prime *}}{\longrightarrow} \mathcal{F} \stackrel{\rho_{*}}{\longleftarrow} \mathcal{G} \stackrel{\delta_{*}^{\prime}}{\longrightarrow} \mathcal{H}$ explicitly, cf. (2.7) and (2.2) (we write $\bar{P}_{i}$ for $P_{i} / f P_{i}$; note that $\operatorname{Hom}_{A}(A / f, A / f)=A / f$ and $\operatorname{Hom}_{A}(A, A / f)=A / f$ and $\operatorname{Hom}_{A}(A, A)=A$ canonically, so that we can for example identify $\operatorname{Hom}_{A}\left(M_{i}, A / f\right)=M_{i}$ and $\operatorname{Hom}\left(P_{0}, A / f\right)=\bar{P}_{0}$; note that some matrix entries are zero since $f: A / f \rightarrow A / f$ is zero; we indicate the transpose of a matrix by an upper index $\mathrm{t}$ ).

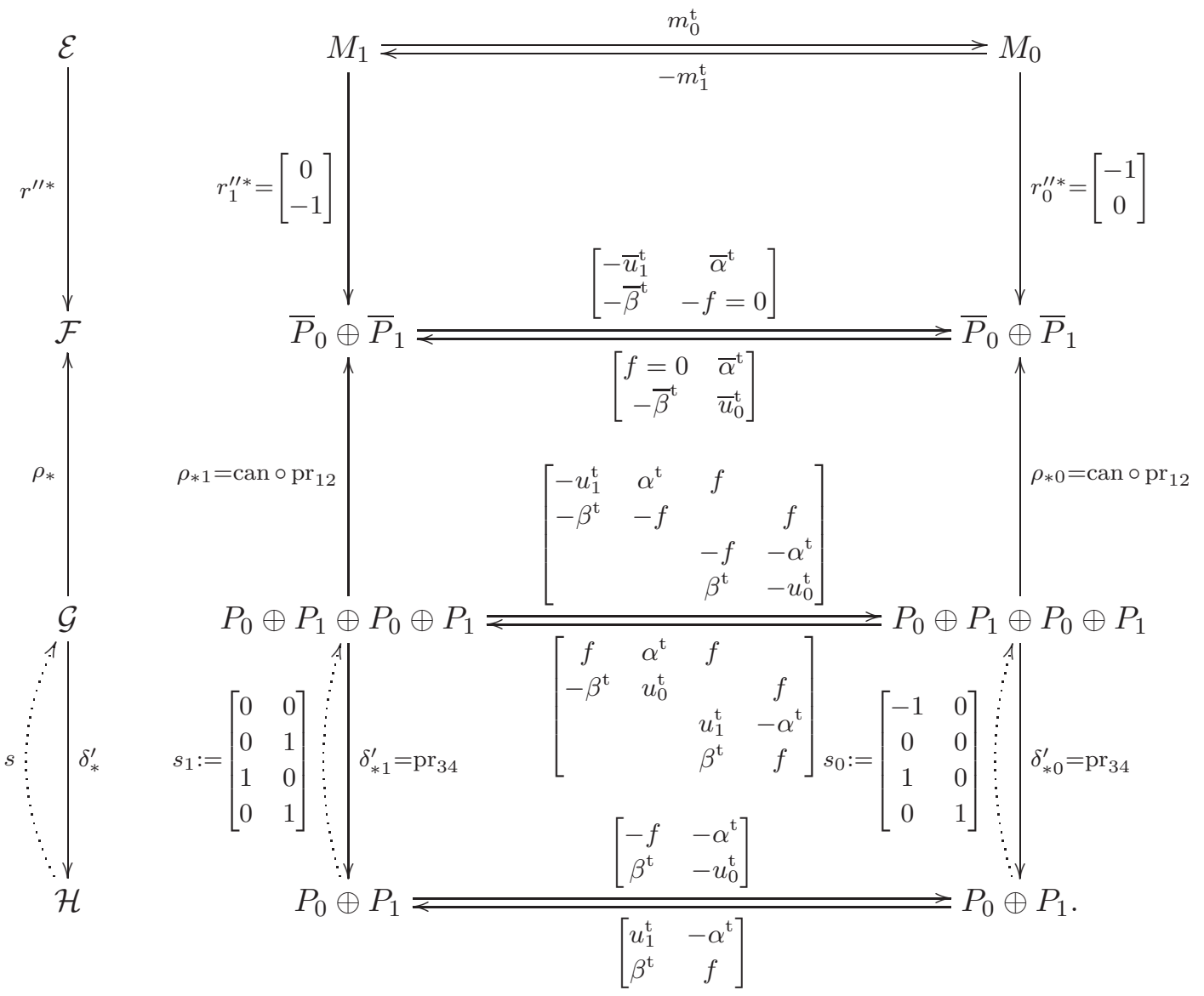

Additionally we have added the dotted morphism $s$ which shows that the surjection $\delta_{*}^{\prime}$ splits in $Z_{0}(\operatorname{Coh}(\operatorname{Spec} A,-W))$. Note that $\rho_{*}$ maps $s(\mathcal{H})$ onto $r^{\prime \prime *}(\mathcal{E})$, so we can consider the commutative diagram

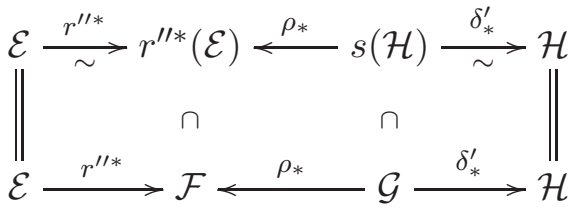


in $Z_{0}(\operatorname{Coh}(\operatorname{Spec} A,-W))$. Our aim is to show that the lower row defines an isomorphism $\delta_{*}^{\prime} \circ\left(\rho_{*}\right)^{-1} \circ r^{\prime \prime *}$ in $\operatorname{DCoh}(\operatorname{Spec} A,-W)$. For this it is clearly sufficient to show that

$$
\mathcal{H} \stackrel{s}{\sim} s(\mathcal{H}) \stackrel{\rho_{*}}{\longrightarrow} r^{\prime \prime *}(\mathcal{E})
$$

becomes an isomorphism in $\operatorname{DCoh}(\operatorname{Spec} A,-W)$. This morphism occurs as the epimorphism in the short exact sequence

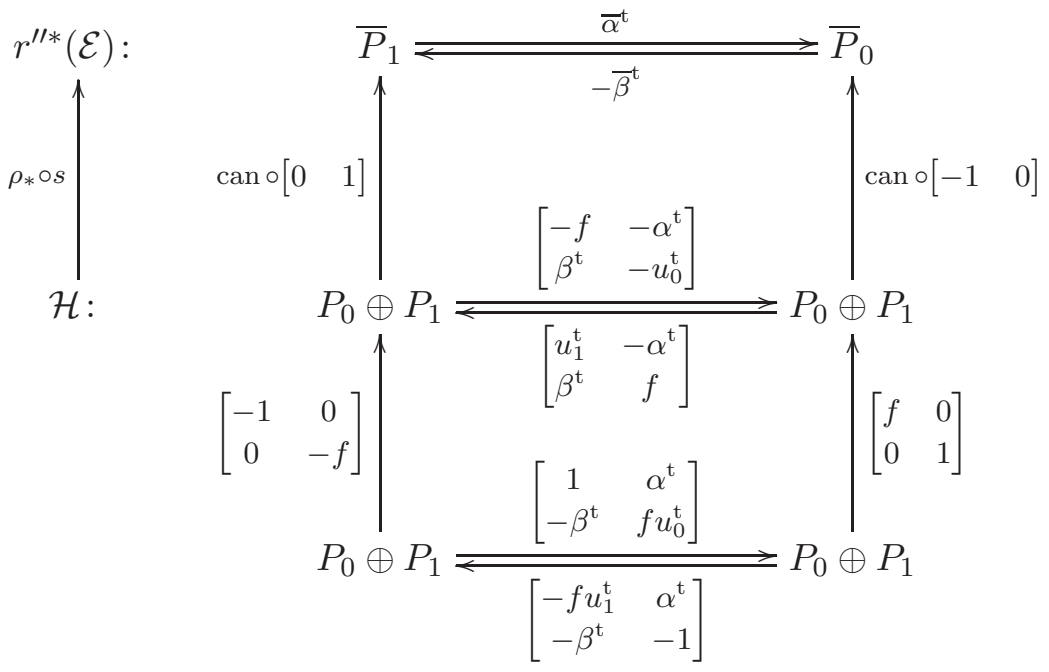

in $Z_{0}(\operatorname{Coh}(\operatorname{Spec} A,-W))$. The lower object in this short exact sequence becomes zero in $[\operatorname{Coh}(\operatorname{Spec} A,-W)]$ (use the homotopy with components $h_{1}=\left[\begin{array}{cc}0 & 0 \\ 0 & -1\end{array}\right]$ and $h_{0}=\left[\begin{array}{ll}1 & 0 \\ 0 & 0\end{array}\right]$ ). This implies that $\rho_{*} \circ s: \mathcal{H} \rightarrow r^{\prime \prime *}(\mathcal{E})$ becomes an isomorphism in $\mathrm{DCoh}(\operatorname{Spec} A,-W)$ and finishes the proof of Proposition 3.7.

Remark 3.8. Lemma 2.43 shows that the subcategory $\pi^{*} \mathbf{M F}(X, W) \subset \mathbf{M F}(\widetilde{X}, W)$ is invariant under the duality $D_{\widetilde{X}}: \mathbf{M F}(\widetilde{X}, W) \rightarrow \mathbf{M F}(\widetilde{X},-W)^{\mathrm{op}}$. This duality takes the subcategory $\mathbf{M F}(Y, W)_{l}$ to the subcategory $\mathbf{M F}(Y,-W)_{-l-1}$, as follows from Proposition 3.7 and Lemma 2.43 again.

Proof of $(M F 2)_{\tilde{X}}$. The method used to prove $(\mathrm{MF} 2)_{E}$ also shows that $\pi^{*} \mathbf{M F}(X, W) \subset$ $\operatorname{MF}(\tilde{X}, W)$ is admissible.

Let us prove that $\mathbf{M F}(Y, W)_{l} \subset \mathbf{M F}(\widetilde{X}, W)$ is admissible.

From the proof of $(\mathrm{MF} 2)_{E}$ it is clear that the functor functor $\mathcal{O}(l) \otimes p^{*}(-): \mathbf{M F}(Y, W) \rightarrow$ $\mathbf{M F}(E, W)$ has a right and a left adjoint functor. Let us view $j_{*}$ as a functor $\mathbf{M F}(E, W) \rightarrow$ $\operatorname{MF}(\widetilde{X}, W)$; it has a left adjoint $j^{*}$ by Lemma 2.37. Proposition 3.7 shows that $F \mapsto$ $D_{E}\left(j^{*}[1](1) D_{\widetilde{X}}(F)\right)$ is right adjoint to $j_{*}$. As above Remark A.5 and its dual show that $\mathbf{M F}(Y, W)_{l} \subset \mathbf{M F}(\widetilde{X}, W)$ is admissible.

Proof of semi-orthogonality in $(M F 3)_{\widetilde{X}}$. Lemma 2.55 shows that this is a direct consequence of Theorem 3.4. $(\mathrm{Coh} 3)_{\widetilde{X}}$ (and this statement is not difficult to prove using the local-to-global Ext spectral sequence).

It remains to prove completeness in $(\mathrm{MF} 3)_{\tilde{X}}$. 
Proposition 3.9. The condition $(M F 3)_{\tilde{X}}$ is equivalent to the following condition $(M F 4)_{\tilde{X}}$ There is a semi-orthogonal decomposition

$$
\mathbf{M F}(\tilde{X},-W)=\left\langle\pi^{*} \mathbf{M F}(X,-W), \mathbf{M F}(Y,-W)_{0}, \ldots, \mathbf{M F}(Y,-W)_{r-2}\right\rangle .
$$

Proof. This follows from Remark 3.8.

This proof of course shows that semi-orthogonality holds in $(\mathrm{MF} 4)_{\widetilde{X}}$ since it holds in $(\mathrm{MF} 3)_{\tilde{X}}$. Hence we have to prove completeness in $(\mathrm{MF} 4)_{\widetilde{X}}$ (obviously we can replace $W$ by $-W$ there). Our first aim is to prove the weaker statement of Proposition 3.11 below.

Lemma 3.10. Let $B \in \mathbf{M F}(\widetilde{X}, W)$.

(a) The condition $B \in{ }^{\perp}\left(\pi^{*} \mathbf{M F}(X, W)\right)$ is equivalent to $\mathbf{R} \pi_{*}\left(D_{\widetilde{X}}(B)\right)=0$. In particular, it is local on $X$ in the following sense: if $\mathcal{U}$ is any open covering of $X$, then $B \in{ }^{\perp}\left(\pi^{*} \mathbf{M F}(X, W)\right)$ if and only if $\left.B\right|_{\pi^{-1}(U)} \in{ }^{\perp}\left(\pi^{*} \mathbf{M F}(U, W)\right)$ for all $U \in \mathcal{U}$.

(b) Let $l \in \mathbb{Z}$. The condition $B \in^{\perp}\left(\mathbf{M F}(Y, W)_{l}\right)$ is local on $X$.

Proof. (a): In somewhat risky notation we have

$$
\begin{aligned}
\operatorname{Hom}_{\mathbf{M F}(\widetilde{X}, W)}\left(B, \pi^{*} \mathbf{M F}(X, W)\right) & =\operatorname{Hom}_{\mathbf{M F}(\widetilde{X},-W)}\left(\pi^{*} \mathbf{M F}(X,-W), D_{\widetilde{X}}(B)\right) \\
& =\operatorname{Hom}_{\mathbf{M F}(X,-W)}\left(\mathbf{M F}(X,-W), \mathbf{R} \pi_{*} D_{\widetilde{X}}(B)\right) .
\end{aligned}
$$

The first equality uses the duality $D_{\widetilde{X}}$ and Remark 3.8, the second equality uses the adjunction of Lemma 2.37. Hence $B \in^{\perp}\left(\pi^{*} \mathbf{M F}(X, W)\right)$ is equivalent to $\mathbf{R} \pi_{*}\left(D_{\widetilde{X}}(B)\right)=0$, and this condition is clearly local on $X$ (use Lemma 2.56).

(b): We have

$$
\begin{aligned}
\operatorname{Hom}_{\mathbf{M F}(\widetilde{X}, W)}\left(B, \mathbf{M F}(Y, W)_{l}\right) & =\operatorname{Hom}_{\mathbf{M F}(E, W)}\left(j^{*} B, \mathcal{O}_{E}(l) \otimes p^{*} \mathbf{M F}(Y, W)\right) \\
& =\operatorname{Hom}_{\mathbf{M F}(E, W)}\left(\mathcal{O}_{E}(-l) \otimes j^{*} B, p^{*} \mathbf{M F}(Y, W)\right) \\
& =\operatorname{Hom}_{\mathbf{M F}(Y,-W)}\left(\mathbf{M F}(Y,-W), \mathbf{R}_{*}\left(D_{E}\left(\mathcal{O}_{E}(-l) \otimes j^{*} B\right)\right)\right)
\end{aligned}
$$

The first equality follows from the adjunction of Lemma 2.37, the second equality is just the twist, and the last equality is obtained similarly as (3.7) (for $p$ instead of $\pi$ ). Clearly the condition $\mathbf{R} p_{*}\left(D_{E}\left(\mathcal{O}_{E}(-l) \otimes j^{*} B\right)\right)=0$ is local on $X$.

Proposition 3.11. The left orthogonal of the full triangulated subcategory

$$
\mathcal{C}:=\operatorname{tria}\left(\pi^{*} \mathbf{M F}(X, W), \mathbf{M F}(Y, W)_{0}, \ldots, \mathbf{M F}(Y, W)_{r-1}\right)
$$

in $\operatorname{MF}(\widetilde{X}, W)$ is zero, ${ }^{\perp} \mathcal{C}=0$.

Proof. Let $B \in{ }^{\perp} \mathcal{C}$. From Lemma 3.10.(a) we obtain $\mathbf{R} \pi_{*}\left(D_{\tilde{X}}(B)\right)=0$. Let $U \subset \widetilde{X}$ be the open complement of $E \subset \widetilde{X}$. Then $\mathbf{R} \pi_{*}\left(D_{U}\left(\left.B\right|_{U}\right)\right)=0$, and the restriction $\left.B\right|_{U}$ is zero in $\operatorname{MF}(U, W)$.

Recall that the line bundle $\mathcal{I}:=\mathcal{O}_{\widetilde{X}}(1) \subset \mathcal{O}_{\widetilde{X}}$ is the ideal sheaf defining $E$. Note that the obvious morphism $B(n)=\mathcal{O}_{\widetilde{X}}(n) \otimes B \rightarrow \mathcal{I}^{n} B$ is an isomorphism for all $n \geq 0$, in particular $\mathcal{I}^{n} B \in \mathbf{M F}(\tilde{X}, W)$. 
Consider for $n \geq 0$ the short exact sequence

$$
0 \rightarrow \mathcal{I}^{n} B \rightarrow B \rightarrow B / \mathcal{I}^{n} B \rightarrow 0
$$

in $Z_{0}(\operatorname{Coh}(\tilde{X}, W))$. Since $\left.B\right|_{U}=0$, Proposition 2.61 shows that $B$ is a direct summand of $B / \mathcal{I}^{n} B$ for $n \gg 0$. Fix $n \gg 0$. It suffices to prove that $B / \mathcal{I}^{n} B=0$ in $\operatorname{DCoh}(\widetilde{X}, W)$.

Since $B \in{ }^{\perp} \mathcal{C}$ the adjunction of Lemma 2.37, implies that

$$
j^{*} B \in^{\perp} \operatorname{tria}\left(p^{*} \mathbf{M F}(Y, W), \ldots, \mathcal{O}(r-1) \otimes p^{*} \mathbf{M F}(Y, W)\right) .
$$

Hence $(\mathrm{MF} 3)_{E}$ implies that $j^{*} B=0$ in $\mathbf{M F}(E, W)$. But then $B / B(1) \cong B / \mathcal{I} B=j_{*} j^{*} B=0$ in $\mathbf{M F}(\widetilde{X}, W)$. Hence $B(1) \rightarrow B$ becomes an isomorphism in $\mathbf{M F}(\widetilde{X}, W)$; the same is then true for $B(n) \rightarrow B$, and hence $0=B / B(n) \cong B / \mathcal{I}^{n} B$ in $\operatorname{DCoh}(\widetilde{X}, W)$.

We give a local description of the inclusion $Y \subset X$ around a closed point $y \in Y$. Let Spec $R$ be an affine open neighborhood of $y$ in $X$, and let $I \subset R$ be the ideal defining $Y \cap \operatorname{Spec} R$. By possibly shrinking $\operatorname{Spec} R$ we can find $r$ elements $x_{1}, \ldots, x_{r} \in R$ that can be extended to a system of uniformizing parameters on $\operatorname{Spec} R$ such that $I=\left(x_{1}, \ldots, x_{r}\right)$ (this follows for example from [Liu02, Cor. 4.2.15] applied to $R$ localized at the maximal ideal corresponding to $y$ ).

In the following subsection 3.2.1 we prove some results, in particular completeness in $(\mathrm{MF} 4)_{\tilde{X}}$, for the local situation $\operatorname{Spec} R / I \subset \operatorname{Spec} R$ just described. In subsection 3.2.2 we then deduce completeness in $(\mathrm{MF} 4)_{\tilde{X}}$ in the global setting.

3.2.1. Local considerations. Let $R$ be a regular Noetherian $k$-algebra (with $\operatorname{Spec} R$ of finite Krull dimension) and let $I \subset R$ be an ideal that is generated by elements $x_{1}, \ldots, x_{r} \in R$ which are part of a system of uniformizing parameters on Spec $R$. Abbreviate $\bar{R}:=R / I$. We assume in this whole subsection 3.2.1 that the inclusion $i: Y \rightarrow X$ is given by $\operatorname{Spec} \bar{R} \subset$ Spec $R$. Then $\widetilde{X}=\operatorname{Proj}\left(R \oplus I \oplus I^{2} \oplus \ldots\right)$, where $R \oplus I \oplus I^{2} \oplus \ldots$ is the Rees algebra of $I \subset R$. We define $y_{a}:=x_{a} \in I \subset R \oplus I \oplus I^{2} \oplus \ldots$, i. e. $y_{a}$ is $x_{a}$ considered as an element of degree 1 in the Rees algebra. Since $x_{1}, \ldots, x_{r}$ is a regular sequence in $R$, the $\bar{R}$-module $I / I^{2}$ is free with basis the images $\bar{y}_{a}$ of the $y_{a}$, and the natural map $\bar{R}\left[\bar{y}_{1}, \ldots, \bar{y}_{r}\right]=\operatorname{Sym}_{R / I}\left(I / I^{2}\right) \rightarrow$ $R / I \oplus I / I^{2} \oplus I^{2} / I^{3} \oplus \ldots$ is an isomorphism. Hence $E=\operatorname{Proj}\left(R / I \oplus I / I^{2} \oplus I^{2} / I^{3} \oplus \ldots\right)=\mathbb{P}_{\bar{R}}^{r-1}$. Let

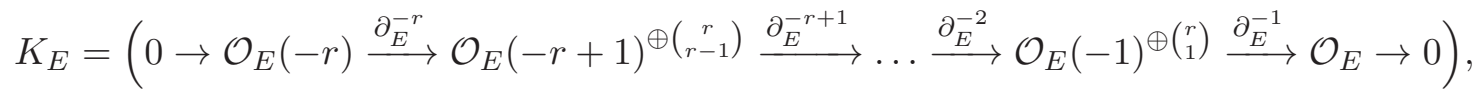

be the acyclic Koszul complex $K_{E}$ on $E$ defined as the following tensor product of complexes,

$$
K_{E}:=\bigotimes_{a=1}^{r}\left(\mathcal{O}_{E}(-1) \stackrel{\bar{y}_{a}}{\rightarrow} \mathcal{O}_{E}\right)
$$

Remark 3.12. The kernel of $\partial_{E}^{-s}$ is canonically isomorphic to the vector bundle $\Omega_{E / Y}^{s}$ (for example $\left.\mathcal{O}_{E}(-r)=\Omega_{E / Y}^{r-1}\right)$. Indeed, it is a nice exercise to show that the complex $K_{E}$ can also be obtained as follows: the dual of the Euler sequence gives rise to several short exact sequences (see [OSS11, I.1.1.(3)]); combining these in the obvious manner yields a long exact sequence which coincides with $K_{E}$. 
Corollary 3.13. For any $s \geq 0$ we have

$$
\Omega_{E / Y}^{s}(s) \otimes p^{*} \mathbf{M F}(Y, W) \subset \operatorname{tria}\left(p^{*} \mathbf{M F}(Y, W), \ldots, \mathcal{O}_{E}(s) \otimes p^{*} \mathbf{M F}(Y, W)\right) .
$$

in $\mathbf{M F}(E, W)$.

Proof. Remark 3.12 provides an acyclic subcomplex of $K_{E}$ which vanishes in degrees < $-s-1$, whose component in degree $-s-1$ is isomorphic to $\Omega_{E / Y}^{s}$, and which coincides with $K_{E}$ in degrees $\geq-s$. Given an object $M \in \mathbf{M F}(Y, W)$, tensor $p^{*}(M)$ with this complex and twist by $\mathcal{O}_{E}(s)$. Now use Lemma 2.7.(c) and the method used to prove part (d) of the same lemma.

Proposition 3.14. The cohomology sheaves of $\mathbf{L} \pi^{*}\left(i_{*} \mathcal{O}_{Y}\right) \in D^{b}(\operatorname{Coh}(\widetilde{X}))$ are given as follows.

(a) $H^{-s}\left(\mathbf{L} \pi^{*}\left(i_{*} \mathcal{O}_{Y}\right)=j_{*} \Omega_{E / Y}^{s}(s)\right.$ for $-s \in[-r+1,0]$;

(b) $H^{t}\left(\mathbf{L} \pi^{*}\left(i_{*} \mathcal{O}_{Y}\right)=0\right.$ for $t \notin[-r+1,0]$.

In fact, for $-s \in[-r+1,0]$, there is an isomorphism

$$
H^{-s}\left(\mathbf{L} \pi^{*}\left(i_{*}(-)\right)\right) \stackrel{\sim}{\rightarrow} j_{*}\left(\Omega_{E / Y}^{s}(s) \otimes p^{*}(-)\right)
$$

of functors free $(Y) \rightarrow \operatorname{Coh}(\tilde{X})$, where free $(Y) \subset \operatorname{Coh}(Y)$ is the full subcategory consisting of free $\mathcal{O}_{Y}$-modules of finite rank, and the functor on the left is the composition

$$
\text { free }(Y) \subset \operatorname{Coh}(Y) \subset D^{b}(\operatorname{Coh}(Y)) \stackrel{i_{*}}{\rightarrow} D^{b}\left(\operatorname{Coh}(X) \stackrel{\mathbf{L} \pi^{*}}{\longrightarrow} D^{b}(\operatorname{Coh}(\widetilde{X})) \stackrel{H^{-s}}{\longrightarrow} \operatorname{Coh}(\widetilde{X}) .\right.
$$

Proof. Consider the Koszul complex $K_{R}:=\left(R ; x_{1}, \ldots, x_{r}\right)$ which is a resolution of $\bar{R}$,

$$
\left(K_{R} \rightarrow \bar{R}\right)=\left(0 \rightarrow R \rightarrow R^{\oplus r} \rightarrow \ldots \rightarrow R^{\oplus\left(\begin{array}{c}
r \\
2
\end{array}\right)} \rightarrow R^{\oplus r} \rightarrow R \rightarrow \bar{R} \rightarrow 0\right)
$$

Then $\mathbf{L} \pi^{*}\left(i_{*} \mathcal{O}_{Y}\right)=\pi^{*}\left(K_{R}\right)$. This already implies that the cohomology sheaves of $\mathbf{L} \pi^{*}\left(i_{*} \mathcal{O}_{Y}\right)$ are zero outside $[-r, 0]$. Note that $K_{R}$ is the tensor product of the complexes $\left(R ; x_{a}\right)=$ $\left(R \stackrel{x_{a}}{\rightarrow} R\right)$, hence

$$
\pi^{*}\left(K_{R}\right)=\bigotimes_{a=1}^{r}\left(\mathcal{O}_{\tilde{X}} \stackrel{x_{a}}{\longrightarrow} \mathcal{O}_{\tilde{X}}\right) .
$$

We will calculate the cohomology of the complex $\pi^{*}\left(K_{R}\right)$ by comparing it to the acyclic Koszul complex

$$
K_{\widetilde{X}}=\left(0 \rightarrow \mathcal{O}_{\widetilde{X}}(-r) \rightarrow \mathcal{O}_{\widetilde{X}}(-r+1)^{\oplus\left(\begin{array}{c}
r \\
r-1
\end{array}\right)} \rightarrow \ldots \rightarrow \mathcal{O}_{\widetilde{X}}(-1)^{\oplus\left(\begin{array}{l}
r \\
1
\end{array}\right)} \rightarrow \mathcal{O}_{\widetilde{X}} \rightarrow 0\right)
$$

which is defined to be the following tensor product,

$$
K_{\widetilde{X}}:=\bigotimes_{a=1}^{r}\left(\mathcal{O}_{\widetilde{X}}(-1) \stackrel{y_{a}}{\rightarrow} \mathcal{O}_{\widetilde{X}}\right) .
$$

Note that the Koszul complex $K_{E}$ above is the restriction $\left.K_{\widetilde{X}}\right|_{E}$ of $K_{\widetilde{X}}$ to the divisor $E$.

Consider the global section $\gamma$ of the line bundle $\mathcal{O}_{\widetilde{X}}(-1)$ defined by $\left.\gamma\right|_{\left\{y_{b} \neq 0\right\}}=\frac{x_{b}}{y_{b}}$ (on the chart $\left\{y_{b} \neq 0\right\}$ ) for $1 \leq b \leq r$. It corresponds to a morphism $\gamma: \mathcal{O}_{\tilde{X}} \rightarrow \mathcal{O}_{\widetilde{X}}(-1)$ (which is 
just the (-1)-twist of the first morphism in (3.3)). The vertical arrows in the commutative square

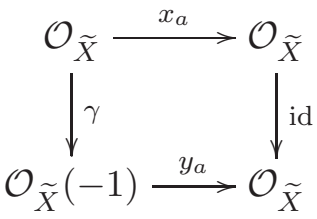

define an injective morphism of two-term complexes indexed by $1 \leq a \leq r$. Their tensor product is an injective morphism $\pi^{*}\left(K_{R}\right) \rightarrow K$. In degree $(-s)$ it is given by the $\left(\begin{array}{l}r \\ s\end{array}\right)$-fold coproduct of the map

$$
\gamma^{\otimes s}: \mathcal{O}_{\widetilde{X}} \rightarrow \mathcal{O}_{\widetilde{X}}(-s)
$$

We denote its cokernel by $\bar{K}$ and obtain a short exact sequence

$$
0 \rightarrow \pi^{*}\left(K_{R}\right) \rightarrow K_{\widetilde{X}} \rightarrow \bar{K} \rightarrow 0
$$

of complexes of sheaves on $\tilde{X}$. Its middle term is acyclic, so $H^{t}\left(\pi^{*}\left(K_{R}\right)\right)=H^{t-1}(\bar{K})$. In particular, it becomes clear that $H^{-r}\left(\mathbf{L} \pi^{*}\left(i_{*} \mathcal{O}_{Y}\right)\right)$ vanishes; hence (b) is proved.

For $n \geq 0$ denote by $E^{n}$ the $n$-th infinitesimal neighborhood of $E$ in $\widetilde{X}$, i. e. the closed subscheme of $\widetilde{X}$ defined by the $(n+1)$-st power of the ideal sheaf $\mathcal{I}=\mathcal{O}_{\widetilde{X}}(1)$ of $E$. The cokernel of the map $\gamma^{\otimes s}: \mathcal{O}_{\widetilde{X}} \rightarrow \mathcal{O}_{\widetilde{X}}(-s)$ is $\mathcal{O}_{E^{s-1}}(-s)$. Hence $\bar{K}^{0}=0$ and $\bar{K}^{-s}=$

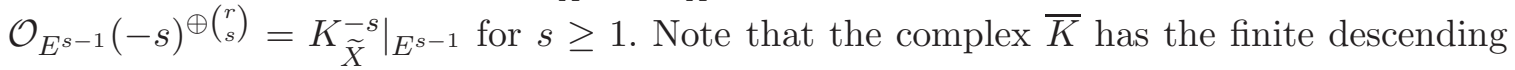
filtration $\bar{K} \supset \mathcal{I} \bar{K} \supset \mathcal{I}^{2} \bar{K} \supset \cdots \supset \mathcal{I}^{r} \bar{K}=0$. We include a picture of

$$
\bar{K}: \quad \ldots \longrightarrow \mathcal{O}_{E^{2}}(-3)^{\oplus}\left(\begin{array}{c}
r \\
3
\end{array}\right) \longrightarrow \mathcal{O}_{E^{1}}(-2)^{\oplus}\left(\begin{array}{c}
r \\
2
\end{array}\right) \longrightarrow \mathcal{O}_{E}(-1)^{\oplus r} \longrightarrow 0,
$$

and of the (non-trivial) associated graded complexes

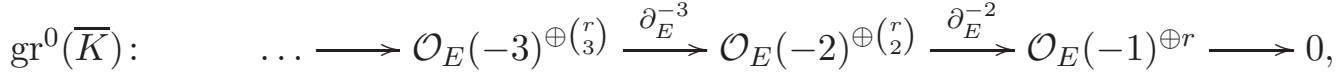

$$
\begin{aligned}
& \operatorname{gr}^{1}(\bar{K}): \quad \ldots \longrightarrow \mathcal{O}_{E}(-2)^{\oplus}\left(\begin{array}{l}
r \\
3
\end{array}\right) \stackrel{\partial_{E}^{-3}(1)}{\longrightarrow} \mathcal{O}_{E}(-1)^{\oplus}\left(\begin{array}{c}
r \\
2
\end{array}\right) \longrightarrow 0, \\
& \operatorname{gr}^{2}(\bar{K}): \quad \ldots \longrightarrow \mathcal{O}_{E}(-1)^{\oplus}\left(\begin{array}{l}
r \\
3
\end{array}\right) \longrightarrow 0 \longrightarrow 0
\end{aligned}
$$

in degrees between -3 and 0 . Remark 3.12 shows that the cohomology of $\operatorname{gr}^{s}(\bar{K})$ is concentrated in degree $-s-1$ and canonically isomorphic to $\operatorname{ker}\left(\partial_{E}^{-s}(s)\right)=\Omega_{E / Y}^{s}(s)$, or more precisely to $j_{*} \Omega_{E / Y}^{s}(s)$.

It is straightforward to see that spectral sequence associated to our filtered complex $\bar{K}$ (whose $E_{0}$-page is $\operatorname{gr}^{*}(\bar{K})$ depicted above, up to a coordinate change) satisfies $E_{1}=E_{2}=$ $\cdots=E_{\infty}$, and that the induced filtration on each $H^{t}(\bar{K})$ has at most one non-trivial subquotient. We hence obtain canonically

$H^{-s}\left(\mathbf{L} \pi^{*} i_{*} \mathcal{O}_{Y}\right)=H^{-s}\left(\pi^{*}\left(K_{R}\right)\right)=H^{-s-1}(\bar{K})=H^{-s-1}\left(\operatorname{gr}^{s}(\bar{K})\right)=\operatorname{ker}\left(\partial_{E}^{-s}(s)\right)=j_{*} \Omega_{E / Y}^{s}(s)$ for $-s \in[-r+1,0]$. This proves (a).

It remains to construct the isomorphism (3.8). Given $M=\bar{R}^{m}$ a free $\bar{R}$-module of finite rank, we take the $m$-fold coproduct of the above construction and obtain an isomorphism 
$H^{-s}\left(\mathbf{L} \pi^{*}\left(i_{*}(M)\right)\right) \stackrel{\sim}{\rightarrow} j_{*}\left(\Omega_{E / Y}^{s}(s) \otimes p^{*}(M)\right)$ in $\operatorname{Coh}(\widetilde{X})$ as the $m$-fold coproduct of (3.9). This defines (3.8) on objects. We claim that this is compatible with morphisms $M \rightarrow N$ in free $(Y)$. It is certainly sufficient to treat the case $M=N=\bar{R}$. Then any morphism $M \rightarrow N$ is given by some $\bar{f} \in \bar{R}$. Choose $f \in R$ with image $\bar{f}$ in $\bar{R}$. Componentwise multiplication with $f$ lifts $f: M=R \rightarrow N=R$ to the Koszul resolution $K_{R}$, and we can use this lift to compute the image of $f$ under $H^{-s}\left(\mathbf{L} \pi^{*}\left(i_{*}(-)\right)\right.$ ) (and this image does not depend on the choice of $f$ since all objects in (3.8) are supported on $E$ ). The image of $f$ under $j_{*}\left(\Omega_{E / Y}^{s}(s) \otimes p^{*}(-)\right)$ is obvious. Now note that all constructions involved in the definition of the isomorphism (3.8) are compatible with multiplication by $f$. This proves our claim.

\section{Corollary 3.15.}

(a) Let $M \in \operatorname{MF}(Y, W)$ and assume that its components $M_{0}$ and $M_{1}$ are free $\bar{R}$-modules of finite rank. Let $0 \rightarrow Q^{n} \rightarrow \cdots \rightarrow Q^{0} \rightarrow i_{*}(M)$ be an exact sequence in $Z_{0}(\operatorname{Coh}(X, W))$ with all $Q^{i} \in \operatorname{MF}(X, W)$, cf. Lemma 2.10.(b). Then the cohomologies of $\pi^{*}(Q)$, considered as a complex in $Z_{0}(\operatorname{Coh}(\widetilde{X}, W))$, are given as follows.

(i) $H^{-s}\left(\pi^{*}(Q)\right) \cong j_{*}\left(\Omega_{E / Y}^{s}(s) \otimes p^{*}(M)\right)$ in $\operatorname{Coh}(\widetilde{X}, W)$, for $-s \in[-r+1,0]$;

(ii) $H^{t}\left(\pi^{*}(Q)\right)=0$ for $t \notin[-r+1,0]$.

(b) We have

$$
\mathbf{M F}(Y, W)_{-1} \subset \operatorname{tria}\left(\pi^{*} \mathbf{M F}(X, W), \mathbf{M F}(Y, W)_{0}, \ldots, \mathbf{M F}(Y, W)_{r-2}\right) .
$$

Proof. (a): The image of the morphisms $m_{0}: M_{0} \rightarrow M_{1}$ and $m_{1}: M_{1} \rightarrow M_{0}$ under the functor $H^{-s}\left(\mathbf{L} \pi^{*}\left(i_{*}(-)\right)\right):$ free $(Y) \rightarrow \operatorname{Coh}(\widetilde{X})$ can be computed using the morphisms $q_{0}: Q_{0} \rightarrow$ $Q_{1}$ and $q_{1}: Q_{1} \rightarrow Q_{0}$ of complexes in $\operatorname{Coh}(X)$. Now use the isomorphism of functors (3.8) (and (b)) in Proposition 3.14.

(b) Let $\mathcal{S}$ be the specified triangulated envelope.

Let $M \in \operatorname{MF}(Y, W)$ have free components, and let $Q \rightarrow i_{*}(M)$ be as in (a). We claim that $j_{*}\left(\mathcal{O}_{E}(-1) \otimes p^{*}(M)\right) \in \mathcal{S}$.

Note that $j_{*}\left(\mathcal{O}_{E}(-1) \otimes p^{*}(M)\right)=j_{*}\left(\Omega_{E / Y}^{r-1}(r-1) \otimes p^{*}(M)\right)$ by Remark 3.12. Hence, by (a), $j_{*}\left(\mathcal{O}_{E}(-1) \otimes p^{*}(M)\right)$ is the $(-r+1)$-st cohomology of the complex $\pi^{*}(Q)$ whose totalization $\mathbf{L} \pi^{*}\left(i_{*} M\right)$ trivially belongs to $\pi^{*} \mathbf{M F}(X, W)$. The other cohomologies of this complex are in the full triangulated subcategory generated by $\mathbf{M F}(Y, W)_{0}, \ldots, \mathbf{M F}(Y, W)_{r-2}$, by part (a) again and Corollary 3.13. The claim follows (by the technique used in the proof of Lemma 2.40.(a)).

Now let $N \in \mathbf{M F}(Y, W)$ be arbitrary. Certainly we find $\bar{R}$-modules $P$ and $Q$ such $N_{0} \oplus P$ and $N_{1} \oplus Q$ are free $\bar{R}$-modules of finite rank. Note that the components of

$$
M:=N \oplus[1] N \oplus(P \underset{W}{\stackrel{1}{\rightleftarrows}} P) \oplus(Q \underset{W}{\stackrel{1}{\rightleftarrows}} Q)
$$

are free $\bar{R}$-modules of finite rank. We already know that $j_{*}\left(\mathcal{O}_{E}(-1) \otimes p^{*}(M)\right) \in \mathcal{S}$. Hence $j_{*}\left(\mathcal{O}_{E}(-1) \otimes p^{*}(N)\right)$ is a direct summand of an object of $\mathcal{S}$. But $\mathcal{S}$ is an admissible subcategory of $\operatorname{MF}(\widetilde{X}, W)$, by Lemma A.9 since $(\mathrm{MF} 2)_{\tilde{X}}$ and semi-orthogonality in $(\mathrm{MF} 4)_{\tilde{X}}$ are already known. In particular, it is a thick subcategory by Corollary A.7. Hence $j_{*}\left(\mathcal{O}_{E}(-1) \otimes p^{*}(N)\right) \in \mathcal{S}$. 
Proof of completeness in $(M F 4)_{\tilde{X}}$ (in the local situation). If we twist the semi-orthogonal decomposition in $(\mathrm{MF} 3)_{E}$ by $\mathcal{O}(r-2)$ we see that

$$
\mathcal{O}(r-1) \otimes p^{*} \mathbf{M F}(Y, W) \subset \operatorname{tria}\left(\mathcal{O}(-1) \otimes p^{*} \mathbf{M F}(Y, W), \ldots, \mathcal{O}(r-2) \otimes p^{*} \mathbf{M F}(Y, W)\right) .
$$

Apply $j_{*}$ to this inclusion. This yields the first inclusion in

$$
\begin{aligned}
\operatorname{MF}(Y, W)_{r-1} & \subset \operatorname{tria}\left(\mathbf{M F}(Y, W)_{-1}, \mathbf{M F}(Y, W)_{0}, \ldots, \mathbf{M F}(Y, W)_{r-2}\right) \\
& \subset \mathcal{D}:=\operatorname{tria}\left(\pi^{*} \mathbf{M F}(X, W), \mathbf{M F}(Y, W)_{0}, \ldots, \mathbf{M F}(Y, W)_{r-2}\right),
\end{aligned}
$$

and the second inclusion follows from Corollary 3.15.(b). This and Proposition 3.11 imply that ${ }^{\perp} \mathcal{D}=0$. Note that $\mathcal{D}$ is admissible by $(\mathrm{MF} 2)_{\tilde{X}}$ and Lemma A.9 since we already know semi-orthogonality in $(\mathrm{MF} 4)_{\tilde{X}}$. But then Remark A.2 shows that $\mathcal{D}=\mathbf{M F}(\widetilde{X}, W)$.

Now the proof of Theorem 3.5 is complete in the local situation described at the beginning of this subsection 3.2.1.

3.2.2. Back to the global setting. We now return to the global blowing-up setting described in subsection 3.2 .

Proof of completeness in $(M F 4)_{\tilde{X}}$ (in the global setting). If $U \subset X$ is an open subscheme, we define $\mathcal{S}_{U}$ to be the subcategory of $\mathbf{M F}\left(\pi^{-1}(U), W\right)$ defined by

$$
\mathcal{S}_{U}:=\operatorname{tria}\left(\pi^{*} \mathbf{M F}(U, W), \mathbf{M F}(Y \cap U, W)_{0}, \ldots, \mathbf{M F}(Y \cap U, W)_{r-2}\right) .
$$

Each $\mathcal{S}_{U}$ is admissible by Lemma A.9 since $(\mathrm{MF} 2)_{\tilde{X}}$ and semi-orthogonality in $(\mathrm{MF} 4)_{\tilde{X}}$ are already known. Let $\mathcal{S}:=\mathcal{S}_{X}$. We need to show that $\mathcal{S}=\operatorname{MF}(\tilde{X}, W)$. By Remark A.2 it suffices to prove that the left orthogonal ${ }^{\perp} \mathcal{S}$ is zero.

Let $B \in{ }^{\perp} \mathcal{S}$. Lemma 3.10 shows that $\left.B\right|_{\pi^{-1}(U)} \in{ }^{\perp}\left(\mathcal{S}_{U}\right)$ for all open $U \subset X$.

Each point of $Y$ has an open neighborhood $U$ in $X$ such that the inclusion $Y \cap U \subset$ $X \cap U$ is isomorphic to $\operatorname{Spec} R / I \subset \operatorname{Spec} R$ with $I \subset R$ as described at the beginning of subsection 3.2.1. Since we already proved $(M F 4)_{\widetilde{X}}$ for this local setting we know that $\mathcal{S}_{U}=\mathbf{M F}\left(\pi^{-1}(U), W\right)$. Hence $\left.B\right|_{\pi^{-1}(U)}=0$ in $\mathbf{M F}\left(\pi^{-1}(U), W\right)$.

Trivially we have $\mathcal{S}_{X \backslash Y}=\mathbf{M F}(\widetilde{X} \backslash E, W)$ and hence $\left.B\right|_{\widetilde{X} \backslash E}=0$ in $\mathbf{M F}(\widetilde{X} \backslash E)$. Now Lemma 2.56 shows that $B=0$ in $\mathbf{M F}(\widetilde{X}, W)$.

This finishes the proof of Theorem 3.5.

We will also need the following lift of this result to the dg level.

Corollary 3.16. In the situation of Theorem 3.5, there is a full $d g$ subcategory $\mathcal{X}$ of $\operatorname{Coh}(\widetilde{X}, W) / \operatorname{AcyclCoh}(\widetilde{X}, W)$ which is quasi-equivalent to $\operatorname{Coh}(X, W) / \operatorname{AcyclCoh}(X, W)$, and there are full $d g$ subcategories $\mathcal{Y}_{l}^{\prime}$ of $\operatorname{Coh}(\widetilde{X}, W) / \operatorname{AcyclCoh}(\widetilde{X}, W)$ (for $\left.l \in \mathbb{Z}\right)$ which are quasi-equivalent to $\operatorname{Coh}(Y, W) / \operatorname{AcyclCoh}(Y, W)$, such that the semi-orthogonal decomposition into admissible subcategories from Theorem 3.5.(MF3) $\widetilde{X}$ is given by

$$
[\operatorname{Coh}(\tilde{X}, W) / \operatorname{AcyclCoh}(\tilde{X}, W)]=\left\langle\left[\mathcal{Y}_{-r+1}^{\prime}\right], \ldots,\left[\mathcal{Y}_{-1}^{\prime}\right],[\mathcal{X}]\right\rangle
$$

if we identify $\operatorname{MF}(\widetilde{X}, W) \stackrel{\sim}{\rightarrow} \operatorname{DCoh}(\widetilde{X}, W)$ with the left-hand side as explained in section 2.6.2. 
Proof. This is proved as Corollary 3.3. We could have used the dg categories Coh(-,?) and $\operatorname{AcyclCoh}(-, ?)$ instead of $\operatorname{MF}(-, ?)$ and $\operatorname{AcyclMF}(-, ?)$ there. Here we need to do this since we have to deal with the functor $j_{*}\left(\mathcal{O}(l) \otimes p^{*}(-)\right)$.

3.3. Applications. Certainly we can apply Theorem 3.2 to $\mathbb{P}_{k}^{n} \rightarrow \operatorname{Spec} k$ and $W=0$. We obtain a semi-orthogonal decomposition of $\mathbf{M F}\left(\mathbb{P}_{k}^{n}, 0\right)$ into admissible subcategories. Let us denote the object $\left(0 \rightleftarrows \mathcal{O}_{\mathbb{P}_{k}^{n}}(i)\right) \in \mathbf{M F}\left(\mathbb{P}_{k}^{n}, 0\right)$ by $\mathcal{O}_{\mathbb{P}_{k}^{n}}(i)$ (by abuse of notation). Then it is not difficult to see that the objects

$$
\mathcal{O}_{\mathbb{P}_{k}^{n}}(-n), \ldots, \mathcal{O}_{\mathbb{P}_{k}^{n}}
$$

define a strong full exceptional collection (in the $\mathbb{Z}_{2}$-graded sense) in $\mathbf{M F}\left(\mathbb{P}_{k}^{n}, 0\right)$. We will explain this in $[\mathrm{Sch}]$ using the folding functor.

We mention some corollaries of Theorem 3.5.

Corollary 3.17. Let $X$ be a scheme satisfying condition ( $r r N f K d$ ) and let $\widetilde{X}$ be the blowingup of $X$ along a regular equi-codimenisonal closed subscheme $Y$ of codimension $r \geq 2$. Let $W: X \rightarrow \mathbb{A}^{1}$ be a morphism.

(a) Assume that $W$ is flat and that the scheme-theoretic zero fiber $X_{0}$ of $W: X \rightarrow \mathbb{A}^{1}$ is regular. Then the category $\mathbf{M F}(\tilde{X}, W)$ has a semi-orthogonal decomposition into $r-$ 1 admissible subcategories that are all equivalent to $\mathbf{M F}(Y, W)$. In particular, if the codimension $r=2$, then $j_{*} p^{*}: \operatorname{MF}(Y, W) \rightarrow \operatorname{DCoh}(\widetilde{X}, W)$ induces an equivalence $\mathbf{M F}(Y, W) \stackrel{\sim}{\rightarrow} \mathbf{M F}(\widetilde{X}, W)$.

(b) Assume that $\left.W\right|_{Y}: Y \rightarrow$ is flat and that its scheme theoretic zero fiber $Y_{0}$ is regular. Then $\pi^{*}: \mathbf{M F}(X, W) \stackrel{\sim}{\rightarrow} \mathbf{M F}(\widetilde{X}, W)$ is an equivalence.

(c) If both $W$ and $\left.W\right|_{Y}$ are flat and have regular scheme-theoretic zero fibers $X_{0}$ and $Y_{0}$, respectively, then $\mathbf{M F}(\tilde{X}, W)=0$.

Proof. Theorem 2.8 shows that $\mathbf{M F}(X, W)=0$ (resp. $\mathbf{M F}(Y, W)=0$ ). All claims then follow from Theorem 3.5.

Example 3.18. Let $X=\mathbb{A}_{k}^{2}=\operatorname{Spec} k[x, y], W=x$ and $Y=\operatorname{Spec} k[x, y] /(x, y)=$ $\{(0,0)\}$. Then Corollary 3.17.(a) shows that $\operatorname{MF}(\operatorname{Spec} k, 0) \stackrel{\sim}{\rightarrow} \operatorname{MF}(\widetilde{X}, W)$. Write $\widetilde{X}=$ Proj $k[x, y][u, v] /(x v-y u)$ and let $U \subset \widetilde{X}$ be the affine open subset defined by $v \neq 0$. Then $U=\operatorname{Spec} k[y, z]=\mathbb{A}_{k}^{2}$ where $z=u / v$, and $W=x=y z$. Theorem 2.8 and [Orl04, Prop. 1.14] imply that $\mathbf{M F}(\widetilde{X}, W) \rightarrow \mathbf{M F}(U, W)$ is an equivalence. Altogether we obtain an equivalence

$$
\mathbf{M F}(\operatorname{Spec} k, 0) \cong \mathbf{M F}\left(\mathbb{A}_{k}^{2}, y z\right)
$$

This is, of course, well known.

Definition 3.19. Let $Z$ be a scheme satisfying condition (srNfKd) and let let $W: Z \rightarrow \mathbb{A}^{1}$ be a regular function. We call $W$ resolved if the ideal sheaf generated by $W$ is locally monomial, i.e. $Z_{0}=\{W=0\}$ is a simple normal crossing divisor. We then also call the corresponding category $\mathrm{MF}(Z, W)$ resolved. 
In the rest of this section we assume in addition that char $k=0$. Let $X$ be a separated connected smooth scheme of finite type and let $W: X \rightarrow \mathbb{A}^{1}$ be a non-zero regular function. By [Kol07, Thm. 3.35] there exists an "embedded resolution of singularities" $\pi: \widetilde{X} \rightarrow X$ of the divisor $X_{0}=\{W=0\}$ such that $W: \widetilde{X} \rightarrow \mathbb{A}^{1}$ is resolved. It is obtained by a sequence of blowing-ups with smooth centers $Y_{1}, \ldots, Y_{s}$ which are contained in the zero sets of the pullbacks of $W$ (as confirmed to us by János Kollár). We can assume that the $Y_{i}$ are connected.

Corollary 3.20. In the above setting the triangulated category $\mathbf{M F}(\widetilde{X}, W)$ has a semiorthogonal decomposition into admissible subcategories that are equivalent to $\mathbf{M F}\left(Y_{i}, 0\right)$ (for $1 \leq i \leq s)$ or $\mathbf{M F}(X, W)$. More precisely, the multiplicity of $\mathbf{M F}\left(Y_{i}, 0\right)$ is equal to the codimension of $Y_{i}$ minus 1 , and $\mathbf{M F}(X, W)$ appears with multiplicity one. In particular, the category $\mathbf{M F}(X, W)$ is a semi-orthogonal summand in a resolved category $\mathbf{M F}(\widetilde{X}, W)$.

Proof. This follows from the above and Theorem 3.5.

Corollary 3.20 may allow us sometimes (depending on the problem we are interested in) to reduce the study of the category $\mathbf{M F}(X, W)$ to the case that the divisor $X_{0}$ is a simple normal crossing divisor. In view of this result we would like to ask the following question.

Question 3.21. Can one give a "reasonable" description of a resolved category $\mathbf{M F}(Z, W)$ ? Or, at least, of its idempotent completion? The simplest non-trivial example would be that of the category $\mathbf{M F}\left(\mathbb{A}^{2}, W=x y^{2}\right)$.

\section{Appendix A. Admissible subCATEgories AND SEMI-ORTHOGONAL DECOMPOSITIONS}

We remind the reader of some definitions and facts from [BK89, BLL04]. Let $\mathcal{T}$ be a triangulated category.

Let $\mathcal{S} \subset \mathcal{T}$ be a subcategory. Recall that the right orthogonal $\mathcal{S}^{\perp}$ to $\mathcal{S}$ in $\mathcal{T}$ is the full subcategory of $\mathcal{T}$ consisting of all objects $C \in \mathcal{T}$ such that $\mathcal{T}(S, C)=0$ for all $S \in \mathcal{S}$. It is a triangulated subcategory of $\mathcal{T}$. Similarly one defines the left orthogonal ${ }^{\perp} \mathcal{S}$.

Definition A.1. A right admissible (resp. left admissible) subcategory of $\mathcal{T}$ is a strict full triangulated subcategory $\mathcal{S}$ of $\mathcal{T}$ such that for any $A \in \mathcal{T}$ there is a triangle $A_{\mathcal{S}} \rightarrow$ $A \rightarrow A_{\mathcal{S}^{\perp}} \rightarrow[1] A_{\mathcal{S}}\left(\right.$ resp. $\left.A_{\perp_{\mathcal{S}}} \rightarrow A \rightarrow A_{\mathcal{S}} \rightarrow[1] A_{\perp_{\mathcal{S}}}\right)$ with $A_{\mathcal{S}} \in \mathcal{S}$ and $A_{\mathcal{S}^{\perp}} \in \mathcal{S}^{\perp}$ (resp. $A_{\perp_{\mathcal{S}}} \in{ }^{\perp} \mathcal{S}$ ). An admissible subcategory is a subcategory which is both right and left admissible.

Remark A.2. Let $\mathcal{S}$ be a right (resp. left) admissible subcategory of $\mathcal{T}$. If $\mathcal{S}^{\perp}=0$ (resp. ${ }^{\perp} \mathcal{S}=0$ ), then obviously $\mathcal{S}=\mathcal{T}$.

Lemma A.3 ([BK89, Prop. 1.5]). Let $\mathcal{S}$ be a strict full triangulated subcategory of a triangulated category $\mathcal{T}$. Then the following are equivalent.

(a) $\mathcal{S}$ is right (resp. left) admissible.

(b) The inclusion functor $\mathcal{S} \hookrightarrow \mathcal{T}$ has a right (resp. left) adjoint.

(c) $\mathcal{T}$ is the triangulated envelope of $\mathcal{S}$ and $\mathcal{S}^{\perp}$ (resp. of ${ }^{\perp} \mathcal{S}$ and $\mathcal{S}$ ). 
Remark A.4. If $\mathcal{S}$ is right (resp. left) admissible and we fix for any $A \in \mathcal{T}$ a triangle $A_{\mathcal{S}} \rightarrow A \rightarrow A_{\mathcal{S}}{ } \rightarrow[1] A_{\mathcal{S}}\left(\right.$ resp. $\left.A_{\perp_{\mathcal{S}}} \rightarrow A \rightarrow A_{\mathcal{S}} \rightarrow[1] A_{\perp_{\mathcal{S}}}\right)$ as above, then $A \mapsto A_{\mathcal{S}}$ extends uniquely to a right (resp. left) adjoint functor to the inclusion $\mathcal{S} \hookrightarrow \mathcal{T}$.

Remark A.5. Let $F: \mathcal{B} \rightarrow \mathcal{T}$ be a full and faithful functor of triangulated categories, and assume that $F$ admits a right adjoint functor. Then the essential image of $F$ is a right admissible subcategory of $\mathcal{T}$. This is obvious from Lemma A.3.

Lemma A.6 (cf. [BLL04, Lemma 2.20]). Let $\mathcal{T}$ be a triangulated category, and let $\mathcal{U}, \mathcal{V}$ be strict full triangulated subcategories of $\mathcal{T}$ satisfying $\mathcal{T}(\mathcal{V}, \mathcal{U})=0$. Assume that there is a full subcategory $\mathcal{E} \subset \mathcal{T}$ such that for each $E \in \mathcal{E}$ there is a triangle

$$
E_{\mathcal{V}} \rightarrow E \rightarrow E_{\mathcal{U}} \rightarrow[1] E_{\mathcal{V}}
$$

with $E_{\mathcal{V}} \in \mathcal{V}$ and $E_{\mathcal{U}} \in \mathcal{U}$. Assume that one of the following two statements is true.

(a) We have tria $(\mathcal{E})=\mathcal{T}$, where tria $(\mathcal{E})$ is the triangulated envelope of $\mathcal{E}$ in $\mathcal{T}$.

(b) The categories $\mathcal{U}$ and $\mathcal{V}$ are thick subcategories of $\mathcal{T}$, one of $\mathcal{U}, \mathcal{V}$ is idempotent complete, and $\operatorname{thick}(\mathcal{E})=\mathcal{T}$, where $\operatorname{thick}(\mathcal{E})$ is the thick envelope of $\mathcal{E}$ in $\mathcal{T}$, i. e. the objects of $\mathcal{E}$ classically generate $\mathcal{T}$.

Then $\mathcal{V}$ is right admissible in $\mathcal{T}, \mathcal{U}$ is left admissible in $\mathcal{T}$, we have $\mathcal{U}=\mathcal{V}^{\perp}$ and $\mathcal{V}={ }^{\perp} \mathcal{U}$, and $\mathcal{T}$ is the triangulated envelope of $\mathcal{U} \cup \mathcal{V}$. In the terminology of Definition A.10 below this says that $\mathcal{T}=\langle\mathcal{U}, \mathcal{V}\rangle$ is a semi-orthogonal decomposition of $\mathcal{T}$.

Proof. Let $\mathcal{S}$ be the full subcategory of $\mathcal{T}$ consisting of those objects $X \in \mathcal{T}$ such that there is a triangle

$$
X_{\mathcal{V}} \rightarrow X \rightarrow X_{\mathcal{U}} \rightarrow[1] X_{\mathcal{V}}
$$

with $X_{V} \in \mathcal{V}$ and $X_{U} \in \mathcal{U}$. We claim that $\mathcal{S}=\mathcal{T}$.

Obviously $\mathcal{S}$ is a strict subcategory containing $\mathcal{E}, \mathcal{V}$ and $\mathcal{U}$, and it is closed under all shifts. Assume that $X \rightarrow Y \rightarrow Z \rightarrow[1] X$ is a triangle with $X, Y \in \mathcal{S}$. Assume that there is a triangle (A.1) as above for $X$, and similarly for $Y$. The morphism $X \rightarrow Y$ extends uniquely two a morphism between these two triangles (use [BBD82, Prop. 1.1.9]), and this morphism fits (since it is unique) into the following $3 \times 3$-diagram constructed using [BBD82, Prop. 1.1.11].

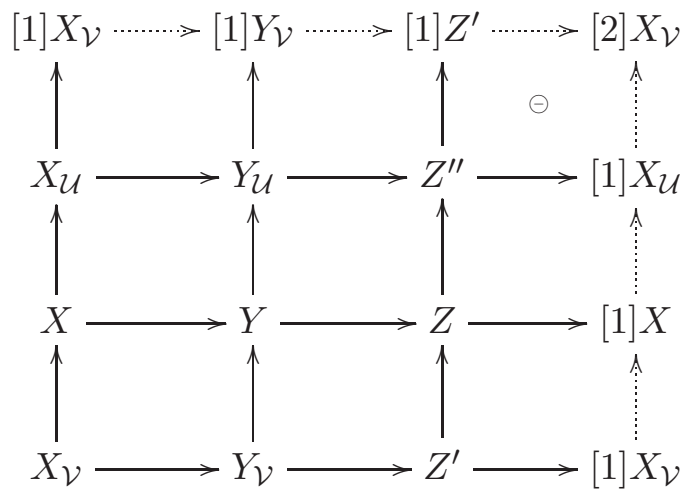


Since $\mathcal{U}$ and $\mathcal{V}$ are strict full triangulated subcategories of $\mathcal{T}$, we have $Z^{\prime} \in \mathcal{V}$ and $Z^{\prime \prime} \in \mathcal{U}$, so $Z \in \mathcal{S}$. This argument shows that $\mathcal{S}$ is a strict triangulated subcategory of $\mathcal{T}$. If (a) is satisfied this already shows that $\mathcal{S}=\mathcal{T}$.

Now assume that (b) is satisfied. We claim that $\mathcal{S}$ is a thick subcategory. Let $X \in \mathcal{S}$ and assume that $X \cong X_{1} \oplus X_{2}$ in $\mathcal{T}$. We can even assume that $X=X_{1} \oplus X_{2}$. Let $V \rightarrow X \rightarrow$ $U \rightarrow[1] V$ be a triangle with $V \in \mathcal{V}$ and $U \in \mathcal{U}$. Then the idempotent $e:=\left[\begin{array}{ll}1 & 0 \\ 0 & 0\end{array}\right]: X \rightarrow X$ can be uniquely extended to a morphism

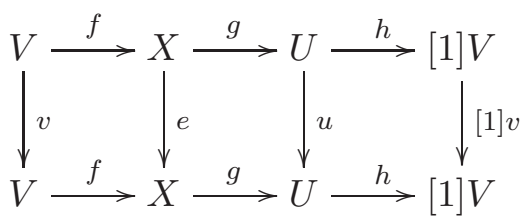

of triangles ([BBD82, Prop. 1.1.9]), and both $u$ and $v$ are idempotent. Assume that $\mathcal{V}$ is idempotent complete. Then we can assume that $V=V_{1} \oplus V_{2}$ with $V_{1}, V_{2} \in \mathcal{V}$ and that $v=\left[\begin{array}{ll}1 & 0 \\ 0 & 0\end{array}\right]$. We have $f=\left[\begin{array}{cc}f_{1} & 0 \\ 0 & f_{2}\end{array}\right]$ since $e f=f v$. Complete the morphisms $f_{i}: V_{i} \rightarrow X_{i}$ into triangles

$$
V_{i} \stackrel{f_{i}}{\rightarrow} X_{i} \rightarrow U_{i} \rightarrow[1] V_{i}
$$

for $i=1,2$. The direct sum of these two triangles is a triangle, and there is a morphism $\varphi$ such that

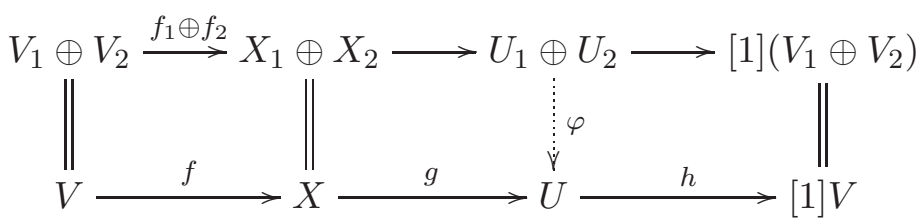

is morphism of triangles; hence $\varphi$ is an isomorphism. Since $\mathcal{U}$ is a thick subcategory, we have $U_{1}, U_{2} \in \mathcal{U}$. The above triangles (A.2) for $i=1,2$ (and the similar argument in case $\mathcal{U}$ is idempotent complete) show that $\mathcal{S}$ is a thick subcategory of $\mathcal{T}$. Hence $\mathcal{S}=\mathcal{T}$.

We have proved that $\mathcal{S}=\mathcal{T}$ if (a) or (b) is satisfied.

By assumption we have $\mathcal{U} \subset \mathcal{V}^{\perp}$. Let $X \in \mathcal{V}^{\perp}$. Since $\mathcal{S}=\mathcal{T}$ we have a triangle

$$
V \rightarrow X \rightarrow U \rightarrow[1] V
$$

with $V \in \mathcal{V}$ and $U \in \mathcal{U}$. Since $X \in \mathcal{V}^{\perp}$ the morphism $V \rightarrow X$ is zero and $\operatorname{id}_{[1] V}$ factors through $U$ (in fact $U \cong X \oplus[1] V)$. But $\mathcal{T}(\mathcal{V}, \mathcal{U})=0$ and hence $[1] V=0$. Hence $X \rightarrow U$ is an isomorphism, and $X \in \mathcal{U}$ by strictness. This shows $\mathcal{U}=\mathcal{V}^{\perp}$. Similarly we obtain $\mathcal{V}={ }^{\perp} \mathcal{U}$

Right admissibility of $\mathcal{V}$, left admissibility of $\mathcal{U}$, and the fact that $\mathcal{T}$ is the triangulated envelope of $\mathcal{U} \cup \mathcal{V}$ follow directly from the definition of $\mathcal{S}$ (cf. (A.1)) and the fact that $\mathcal{S}=\mathcal{T}$.

Corollary A.7 ([BK89, Lemma 1.7]). If $\mathcal{S}$ is a right admissible subcategory of a $\mathcal{T}$, then $\mathcal{S}={ }^{\perp}\left(\mathcal{S}^{\perp}\right)$, so in particular $\mathcal{S}$ is a thick subcategory of $\mathcal{T}$. Similarly, if $\mathcal{S}$ is left admissible, then $\mathcal{S}=\left({ }^{\perp} \mathcal{S}\right)^{\perp}$ is thick. 
Proof. The first statement follows from Lemma A.6 by taking $\mathcal{U}=\mathcal{S}^{\perp}, \mathcal{V}=\mathcal{S}$ and $\mathcal{E}=\mathcal{T}$. For the second statement take $\mathcal{U}=\mathcal{S}, \mathcal{V}={ }^{\perp} \mathcal{S}$ and $\mathcal{E}=\mathcal{T}$.

Lemma A.8. If $\mathcal{S}$ is right admissible, the functors $\mathcal{S}^{\perp} \rightarrow \mathcal{T} / \mathcal{S}$ and $\mathcal{S} \rightarrow \mathcal{T} / \mathcal{S}^{\perp}$ are equivalences. If $\mathcal{S}$ is left admissible, the functors $\mathcal{S} \rightarrow \mathcal{T} /{ }^{\perp} \mathcal{S}$ and ${ }^{\perp} \mathcal{S} \rightarrow \mathcal{T} / \mathcal{S}$ are equivalences.

Proof. By parts (ff2) and (ff2) op of Proposition B.2, all these functors are full and faithful, and it is clear that they are essentially surjective.

Lemma A.9. Let $\mathcal{S}_{1}, \mathcal{S}_{2}$ be right admissible subcategories of a triangulated category $\mathcal{T}$ and assume that $\mathcal{T}\left(\mathcal{S}_{2}, \mathcal{S}_{1}\right)=0$. Then the triangulated envelope $\mathcal{D}:=\operatorname{tria}\left(\mathcal{S}_{1}, \mathcal{S}_{2}\right)$ in $\mathcal{T}$ of the full subcategory $\mathcal{S}_{1} \cup \mathcal{S}_{2}$ is a right admissible subcategory of $\mathcal{T}$.

Similarly, if $\mathcal{S}_{1}$ and $\mathcal{S}_{2}$ are left admissible subcategories of $\mathcal{T}$ satisfying $\mathcal{T}\left(\mathcal{S}_{2}, \mathcal{S}_{1}\right)=0$, then tria $\left(\mathcal{S}_{1}, \mathcal{S}_{2}\right)$ is left admissible in $\mathcal{T}$.

Proof. Let $T \in \mathcal{T}$ be given. By right admissibility of $\mathcal{S}_{2}$ there is a triangle

$$
S_{2} \rightarrow T \stackrel{g_{2}}{\rightarrow} Q_{2} \rightarrow[1] S_{2}
$$

with $S_{2} \in \mathcal{S}_{2}$ and $Q_{2} \in \mathcal{S}_{2}^{\perp}$, and right admissibility of $\mathcal{S}_{1}$ yields a triangle

$$
S_{1} \rightarrow Q_{2} \stackrel{g_{1}}{\rightarrow} Q_{1} \rightarrow[1] S_{1}
$$

with $S_{1} \in \mathcal{S}_{1}$ and $Q_{1} \in \mathcal{S}_{1}^{\perp}$. Note that $S_{1} \in \mathcal{S}_{1} \subset \mathcal{S}_{2}^{\perp}$ and $Q_{2} \in \mathcal{S}_{2}^{\perp}$ imply that $Q_{1} \in \mathcal{S}_{2}^{\perp}$. Hence $Q_{1} \in \mathcal{D}^{\perp}$. Fit the composition $g_{1} g_{2}$ into a triangle

$$
U \rightarrow T \stackrel{g_{1} g_{2}}{\longrightarrow} Q_{1} \rightarrow[1] U
$$

The octahedral axiom applied to the morphisms $g_{2}$ and $g_{1}$ provides a triangle

$$
S_{2} \rightarrow U \rightarrow S_{1} \rightarrow[1] S_{2}
$$

This shows that $U \in \mathcal{D}$. Hence we see from (A.3) that $\mathcal{D}$ is right admissible.

Definition A.10. A sequence $\left(\mathcal{S}_{1}, \mathcal{S}_{2}, \ldots \mathcal{S}_{n}\right)$ of subcategories of $\mathcal{T}$ is called semi-orthogonal if $\mathcal{T}\left(\mathcal{S}_{j}, \mathcal{S}_{i}\right)=0$ for all $j>i$, and complete (in $\mathcal{T}$ ) if $\mathcal{T}$ is the triangulated envelope of $\mathcal{S}_{1} \cup \mathcal{S}_{2} \cup \cdots \cup \mathcal{S}_{n}$. A semi-orthogonal decomposition of $\mathcal{T}$ is a complete semi-orthogonal sequence $\left(\mathcal{S}_{1}, \mathcal{S}_{2}, \ldots \mathcal{S}_{n}\right)$ of strict full triangulated subcategories, and is denoted by

$$
\mathcal{T}=\left\langle\mathcal{S}_{1}, \ldots, \mathcal{S}_{n}\right\rangle .
$$

A semi-orthogonal decomposition into admissible subcategories is a semi-orthogonal decomposition whose components are admissible subcategories.

\section{Lemma A.11.}

(a) If $\mathcal{S}$ is a right admissible subcategory of $\mathcal{T}$, then $\mathcal{T}=\left\langle\mathcal{S}^{\perp}, \mathcal{S}\right\rangle$ is a semi-orthogonal decomposition of $\mathcal{T}$. Similarly, if $\mathcal{S}$ is left admissible, then $\left\langle\mathcal{S},{ }^{\perp} \mathcal{S}\right\rangle$ is a semi-orthogonal decomposition.

(b) If $\mathcal{T}=\langle\mathcal{U}, \mathcal{V}\rangle$ is a semi-orthogonal decomposition, then $\mathcal{V}$ is right admissible, $\mathcal{U}$ is left admissible, $\mathcal{U}=\mathcal{V}^{\perp}$ and $\mathcal{V}={ }^{\perp} \mathcal{U}$. 
(c) Let $\mathcal{T}=\left\langle\mathcal{S}_{1}, \ldots, \mathcal{S}_{n}\right\rangle$ be a semi-orthogonal decomposition (into admissible subcategories $)$, and let $1 \leq a<n$. Let $\mathcal{D}_{1}:=\operatorname{tria}\left(\mathcal{S}_{1} \cup \cdots \cup \mathcal{S}_{a}\right)$ and $\mathcal{D}_{2}:=\operatorname{tria}\left(\mathcal{S}_{a+1} \cup\right.$ $\left.\cdots \cup \mathcal{S}_{n}\right)$ denote the indicated triangulated envelopes. Then $\mathcal{T}=\left\langle\mathcal{D}_{1}, \mathcal{D}_{2}\right\rangle$ and $\mathcal{D}_{1}=\left\langle\mathcal{S}_{1}, \ldots, \mathcal{S}_{a}\right\rangle$ and $\mathcal{D}_{2}=\left\langle\mathcal{S}_{a+1}, \ldots, \mathcal{S}_{n}\right\rangle$ are semi-orthogonal decompositions (into admissible subcategories). In particular, $\mathcal{D}_{1}=\mathcal{D}_{2}^{\perp}$ and $\mathcal{D}_{2}={ }^{\perp} \mathcal{D}_{1}$.

Proof. (a): Use Lemma A.3.

(b): This is a consequence Lemma A.6: take $\mathcal{E}=\mathcal{S}_{1} \cup \mathcal{S}_{2}$.

(c): If $\mathcal{T}=\left\langle\mathcal{S}_{1}, \ldots, \mathcal{S}_{n}\right\rangle$ is a semi-orthogonal decomposition, all statements are trivial (the last one follows directly from (b)). So let us assume that all components $\mathcal{S}_{i}$ are admissible in $\mathcal{T}$. Then Lemma A.9 implies that $\mathcal{D}_{1}$ and $\mathcal{D}_{2}$ are admissible subcategories of $\mathcal{T}$. Moreover, each $\mathcal{S}_{j}$, for $1 \leq j \leq a$ (resp. $\left.a+1 \leq j \leq n\right)$, is obviously admissible in $\mathcal{D}_{1}$ (resp. $\mathcal{D}_{2}$ ).

Corollary A.12. A semi-orthogonal decomposition $\mathcal{T}=\langle\mathcal{U}, \mathcal{V}\rangle$ (into admissibles) induces a semi-orthogonal decomposition (into admissibles) of the Karoubi envelope $\mathcal{T}^{\natural}$ of $\mathcal{T}$, namely $\mathcal{T}^{\natural}=\left\langle\mathcal{U}^{\natural}, \mathcal{V}^{\natural}\right\rangle$.

Proof. Use Lemmata A.6.(b) and A.11.

\section{Appendix B. Embeddings of Verdier quotients}

Verdier localization is described beautifully in [Nee01, 2.1]. We give here some additional results. In contrast to [Nee01] we do not assume that triangulated subcategories are strict (= closed under isomorphisms).

Let $\mathcal{D}$ be a triangulated category and $\mathcal{C} \subset \mathcal{D}$ a full triangulated subcategory (not necessarily thick). Let $F: \mathcal{D} \rightarrow \mathcal{D} / \mathcal{C}$ be the Verdier localization functor ([Nee01, Theorem 2.1.8]). We denote by $\operatorname{Mor}_{\mathcal{C}}$ the subclass of morphisms (in $\mathcal{D}$ ) that fit into a triangle with cone in $\mathcal{C}$.

Lemma B.1. Let $f, g: X \rightarrow Y$ be two morphisms in $\mathcal{D}$. The following conditions are equivalent:

(a) $F(f)=F(g)$;

(b) there is a morphism $\alpha: X^{\prime} \rightarrow X$ in $\operatorname{Mor}_{\mathcal{C}}$ such that $f \alpha=g \alpha: X^{\prime} \rightarrow Y$;

(c) there is a morphism $\beta: Y \rightarrow Y^{\prime}$ in $\operatorname{Mor}_{\mathcal{C}}$ such that $\beta f=\beta g: X \rightarrow Y^{\prime}$;

(d) the morphism $f-g: X \rightarrow Y$ factors as $X \rightarrow C \rightarrow Y$ with $C$ in $\mathcal{C}$.

Proof. This is a slightly extended version of [Nee01, Lemma 2.1.26] using the description of morphisms in $\mathcal{D} / \mathcal{C}$ via "coroofs". The proof is easily generalized.

Proposition B.2. Let $\mathcal{D}$ be a triangulated category with full triangulated subcategories $\mathcal{C}$, $\mathcal{W}, \mathcal{V}$ such that $\mathcal{V}$ is contained in both $\mathcal{W}$ and $\mathcal{C}$, i. e. pictorially

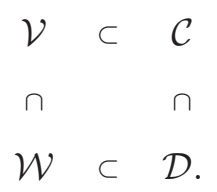


Let $i$ be the inclusion $\mathcal{W} \subset \mathcal{D}$. Then $i$ factors to a triangulated functor $\bar{i}: \mathcal{W} / \mathcal{V} \rightarrow \mathcal{D} / \mathcal{C}$, i. e. pictorially

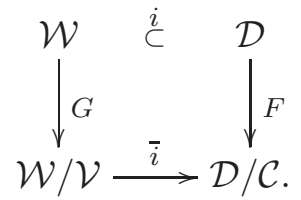

where $F$ and $G$ are the Verdier localization functors.

(I) The following three conditions are equivalent, and if they hold, the functor $\bar{i}$ is full and faithful.

(ff1) For all morphisms $s: W \rightarrow D$ in $\operatorname{Mor}_{\mathcal{C}}$ with $W$ in $\mathcal{W}$ and $D$ in $\mathcal{D}$ there is an object $W^{\prime}$ in $\mathcal{W}$ and a morphism $t: D \rightarrow W^{\prime}$ such that the morphism ts: $W \rightarrow$ $W^{\prime}$ in $\mathcal{W}$ is in $\operatorname{Mor}_{\mathcal{V}}$.

(ff2) Any morphism $C \rightarrow W$ with $C \in \mathcal{C}$ and $W \in \mathcal{W}$ factors as $C \rightarrow V \rightarrow W$ with $V \in \mathcal{V}$.

(Equivalently: For any morphism $s: C \rightarrow W$ with $C \in \mathcal{C}$ and $W \in \mathcal{W}$ there is an object $W^{\prime} \in \mathcal{W}$ and a morphism $t: W \rightarrow W^{\prime}$ in $\operatorname{Mor}_{\mathcal{V}}$ such that $t s=0$.)

(ff3) For all $D \in \mathcal{D}$ and $W \in \mathcal{W}$ the obvious morphism

$$
j: \operatorname{Hom}_{\mathcal{D} / \mathcal{V}}(D, W) \rightarrow \operatorname{Hom}_{\mathcal{D} / \mathcal{C}}(D, W)
$$

is bijective.

These three conditions hold if the following condition (ff4) is satisfied.

$(f f 4) \mathcal{C}$ is classically generated by a collection $\mathcal{E}$ of objects in $\mathcal{D}$, i. e. $\mathcal{C}=\operatorname{thick}(\mathcal{E})$, and any morphism $E \rightarrow W$ with $E \in \mathcal{E}$ and $W \in \mathcal{W}$ factors through an object of $\mathcal{V}$.

(II) Dually, the following three conditions are equivalent, and if they hold, the functor $\bar{i}$ is full and faithful.

(ff1) ${ }^{\text {op }}$ For all morphisms $s: D \rightarrow W$ in $\operatorname{Mor}_{\mathcal{C}}$ with $D$ in $\mathcal{D}$ and $W$ in $\mathcal{W}$ there is an object $W^{\prime}$ in $\mathcal{W}$ and a morphisms $t: W^{\prime} \rightarrow D$ such that the morphism

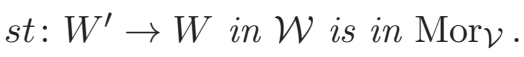

(ff2) $)^{\mathrm{op}}$ Any morphism $W \rightarrow C$ with $W \in \mathcal{W}$ and $C \in \mathcal{C}$ factors as $W \rightarrow V \rightarrow C$ with $V \in \mathcal{V}$.

(Equivalently: For any morphism $s: W \rightarrow C$ with $W \in \mathcal{W}$ and $C \in \mathcal{C}$ there is an object $W^{\prime} \in \mathcal{W}$ and a morphism $t: W^{\prime} \rightarrow W$ in Mor $\mathcal{V}$ such that st =0.)

(ff3) ${ }^{\mathrm{op}}$ For all $W \in \mathcal{W}$ and $D \in \mathcal{D}$ the obvious morphism

$$
\operatorname{Hom}_{\mathcal{D} / \mathcal{V}}(W, D) \rightarrow \operatorname{Hom}_{\mathcal{D} / \mathcal{C}}(W, D)
$$

is bijective.

Moreover, these three conditions hold if the following condition (ff4) op is satisfied.

$(f f 4)^{\mathrm{op}} \mathcal{C}$ is classically generated by a collection $\mathcal{E}$ of objects in $\mathcal{D}$, i. e. $\mathcal{C}=\operatorname{thick}(\mathcal{E})$, and any morphism $W \rightarrow E$ with $W \in \mathcal{W}$ and $E \in \mathcal{E}$ factors through an object of $\mathcal{V}$.

Proof. We use implicitly some results of [Nee01], e. g. Remark 2.1.23. Let $F^{\prime}: \mathcal{D} \rightarrow \mathcal{D} / \mathcal{V}$ be the Verdier localization functor and $j: \mathcal{D} / \mathcal{V} \rightarrow \mathcal{D} / \mathcal{C}$ the functor such that $j F^{\prime}=F$. 
We start with the proof of (I).

(ff1) implies (ff3): Let $D \in \mathcal{D}$ and $W \in \mathcal{W}$. We have to prove that (B.1) is bijective.

Injectivity: Let $h: D \rightarrow W$ be a morphism in $\mathcal{D} / \mathcal{V}$. Then $h=F^{\prime}(f) F^{\prime}(g)^{-1}$ for some $D^{\prime}$ in $\mathcal{D}$ and morphisms $D \stackrel{g}{\leftarrow} D^{\prime} \stackrel{f}{\rightarrow} W$ (a "roof") in $\mathcal{D}$ with $g \in$ Mor $\mathcal{V}$.

Assume that $j(h)=0$. Then $F(f) F(g)^{-1}=0$ and hence $F(f)=0$; it is sufficient to show that $F^{\prime}(f)=0$. Lemma B.1 shows that there is $s: W \rightarrow D^{\prime \prime}$ in Mor $_{\mathcal{C}}$ such that $s f=0: D^{\prime} \rightarrow D^{\prime \prime}$. Assumption (ff1) applied to $s$ yields $W^{\prime}$ in $\mathcal{W}$ and $t: D^{\prime \prime} \rightarrow W^{\prime}$ such that $t s: W \rightarrow W^{\prime}$ is in Mor $\mathcal{V}$. We obtain that $0=t s f: D^{\prime} \stackrel{f}{\rightarrow} W \stackrel{t s}{\rightarrow} W^{\prime}$. This implies $0=$ $F^{\prime}((t s) f)=F^{\prime}(t s) F^{\prime}(f)$. Note that $F^{\prime}(t s)$ is invertible since $t s \in$ Mor $\mathcal{V}$. Hence $F^{\prime}(f)=0$.

Surjectivity: Let a morphism $a: D \rightarrow W$ in $\mathcal{D} / \mathcal{C}$ be represented by a "coroof"

$$
D \stackrel{f}{\rightarrow} D^{\prime} \stackrel{s}{\leftarrow} W
$$

with $s \in \operatorname{Mor}_{\mathcal{C}}$. Assumption (ff1) applied to $s$ yields $W^{\prime}$ in $\mathcal{W}$ and $t: D^{\prime} \rightarrow W^{\prime}$ such that $t s \in \operatorname{Mor}_{\mathcal{V}}$. Our coroof is equivalent to the coroof

$$
D \stackrel{t f}{\longrightarrow} W^{\prime} \stackrel{t s}{\longleftarrow} W
$$

which represents a morphism $b: D \rightarrow W$ in $\mathcal{D} / \mathcal{V}$, namely $b=F^{\prime}(t s)^{-1} F^{\prime}(t f)$. Since $s$ and $t s$ are in $\operatorname{Mor}_{\mathcal{C}}$ the same is true for $t$ by the octahedral axiom. Hence

$$
j(b)=F(t s)^{-1} F(t f)=(F(t) F(s))^{-1} F(t) F(f)=F(s)^{-1} F(f)=a .
$$

(ff3) implies (ff2): Let a morphism $C \rightarrow W$ with $C \in \mathcal{C}$ and $W \in \mathcal{W}$ be given. It becomes zero in $\mathcal{D} / \mathcal{C}$ by Lemma B.1. By assumption it then becomes already zero in $\mathcal{D} / \mathcal{V}$. Lemma B.1 implies that $C \rightarrow W$ factors through $\mathcal{V}$.

(ff2) implies (ff1): Let a morphism $s: W \rightarrow D$ in $\operatorname{Mor}_{\mathcal{C}}$ with $W$ in $\mathcal{W}$ and $D$ in $\mathcal{D}$ be given. Fit $s$ into a triangle $W \stackrel{s}{\rightarrow} D \rightarrow C \rightarrow[1] W$ with $C \in \mathcal{C}$. By assumption $C \rightarrow[1] W$ factors as $C \rightarrow V \rightarrow[1] W$ with $V \in \mathcal{V}$. We fit the morphism $V \rightarrow[1] W$ into a triangle $W \rightarrow W^{\prime} \rightarrow V \rightarrow[1] W$ with $W^{\prime} \in \mathcal{W}$. The partial morphism

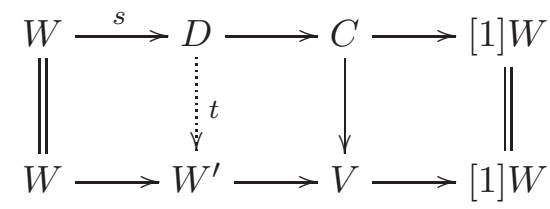

can be completed by a morphism $t$ to a morphism of triangles, and the morphism $t s$ is the first morphism in the lower triangle and hence lies in Mor $\mathcal{V}$.

(ff4) implies (ff2): A morphism $C \rightarrow W$ with $C \in \mathcal{C}$ and $W \in \mathcal{W}$ factors through an object of $\mathcal{V}$ if and only if $C \rightarrow W$ becomes the zero morphism in $\mathcal{D} / \mathcal{V}$, by Lemma B.1. Using this one proves that the class of all objects $E^{\prime}$ such that each morphism from $E^{\prime}$ to an arbitrary object of $\mathcal{W}$ factors through an object of $\mathcal{V}$ is closed under shifts, extensions and direct summands. This implies the claim.

(ff3) implies that $\bar{i}$ is full and faithful: Let $W^{\prime}, W \in \mathcal{W}$. Since $\bar{i}$ factors as $\mathcal{W} / \mathcal{V} \rightarrow$ $\mathcal{D} / \mathcal{V} \rightarrow \mathcal{D} / \mathcal{C}$ it is enough to show that

$$
\operatorname{Hom}_{\mathcal{W} / \mathcal{V}}\left(W^{\prime}, W\right) \rightarrow \operatorname{Hom}_{\mathcal{D} / \mathcal{V}}\left(W^{\prime}, W\right)
$$


is bijective. If $W^{\prime} \stackrel{s}{\leftarrow} D \stackrel{f}{\rightarrow} W$ is a roof with $D \in \mathcal{D}$ and $s \in$ Morv representing a morphism in $\operatorname{Hom}_{\mathcal{D} / \mathcal{V}}\left(W^{\prime}, W\right)$, then $D \stackrel{s}{\rightarrow} W^{\prime}$ fits into a triangle with cone in $\mathcal{V} \subset \mathcal{W}$. The second and third object of this triangle are in $\mathcal{W}$, so the first object $D$ is isomorphic to an object $W^{\prime \prime}$ of $\mathcal{W}$. Let $t: W^{\prime \prime} \stackrel{\sim}{\rightarrow} D$ be an isomorphism. Then our roof is isomorphic to the roof $W^{\prime} \stackrel{s t}{\leftarrow} W^{\prime \prime} \stackrel{f t}{\longrightarrow} W$. This argument shows that the above map is surjective as well as injective.

We leave the proof of the "dual" statements in (II) to the reader.

\section{REFERENCES}

[Aut] The Stacks Project Authors, Stacks Project, http://math.columbia.edu/algebraic_geometry/stacks-git.

[Bas63] Hyman Bass, Big projective modules are free, Illinois J. Math. 7 (1963), 24-31.

[BBD82] A. A. Beŭlinson, J. Bernstein, and P. Deligne, Faisceaux pervers, Analysis and topology on singular spaces, I (Luminy, 1981), Astérisque, vol. 100, Soc. Math. France, Paris, 1982, pp. 5171.

[Bit04] Franziska Bittner, The universal Euler characteristic for varieties of characteristic zero, Compos. Math. 140 (2004), no. 4, 1011-1032.

[BK89] A. I. Bondal and M. M. Kapranov, Representable functors, Serre functors, and reconstructions/mutations, Izv. Akad. Nauk SSSR Ser. Mat. 53 (1989), no. 6, 1183-1205, 1337.

[BLL04] Alexey I. Bondal, Michael Larsen, and Valery A. Lunts, Grothendieck ring of pretriangulated categories, Int. Math. Res. Not. (2004), no. 29, 1461-1495.

[BN93] Marcel Bökstedt and Amnon Neeman, Homotopy limits in triangulated categories, Compositio Math. 86 (1993), no. 2, 209-234.

[BO95] A. Bondal and D. Orlov, Semiorthogonal decomposition for algebraic varieties, arXiv:alg-geom/9506012 (1995).

[BvdB03] A. Bondal and M. van den Bergh, Generators and representability of functors in commutative and noncommutative geometry, Mosc. Math. J. 3 (2003), no. 1, 1-36, 258.

[Dri04] Vladimir Drinfeld, DG quotients of DG categories, J. Algebra 272 (2004), no. 2, 643-691.

[God73] Roger Godement, Topologie algébrique et théorie des faisceaux, Hermann, Paris, 1973.

[Gro61] A. Grothendieck, Éléments de géométrie algébrique. III. Étude cohomologique des faisceaux cohérents (première partie), Inst. Hautes Études Sci. Publ. Math. (1961), no. 11, 167.

[GW10] Ulrich Görtz and Torsten Wedhorn, Algebraic geometry I, Advanced Lectures in Mathematics, Vieweg + Teubner, Wiesbaden, 2010.

[Har66] Robin Hartshorne, Residues and duality, Lecture notes of a seminar on the work of A. Grothendieck, given at Harvard 1963/64. With an appendix by P. Deligne. Lecture Notes in Mathematics, No. 20, Springer-Verlag, Berlin, 1966.

[Har77] _ Algebraic geometry, Springer-Verlag, New York, 1977, Graduate Texts in Mathematics, No. 52 .

[Huy06] D. Huybrechts, Fourier-Mukai transforms in algebraic geometry, Oxford Mathematical Monographs, The Clarendon Press Oxford University Press, Oxford, 2006.

[Kol07] János Kollár, Lectures on resolution of singularities, Annals of Mathematics Studies, vol. 166, Princeton University Press, Princeton, NJ, 2007.

[KS94] Masaki Kashiwara and Pierre Schapira, Sheaves on manifolds, Grundlehren der Mathematischen Wissenschaften [Fundamental Principles of Mathematical Sciences], vol. 292, Springer-Verlag, Berlin, 1994.

[Liu02] Qing Liu, Algebraic geometry and arithmetic curves, Oxford Graduate Texts in Mathematics, vol. 6, Oxford University Press, Oxford, 2002.

[LO10] Valery A. Lunts and Dmitri O. Orlov, Uniqueness of enhancement for triangulated categories, J. Amer. Math. Soc. 23 (2010), no. 3, 853-908.

[LP11] Kevin H. Lin and Daniel Pomerleano, Global matrix factorizations, Preprint (2011), arXiv:arXiv:1101.5847. 
[LSa] Valery A. Lunts and Olaf M. Schnürer, in preparation.

[LSb] Matrix factorizations and motivic measures, in preparation.

[Lun10] Valery A. Lunts, Categorical resolution of singularities, J. Algebra 323 (2010), no. 10, 29773003.

[Mat89] Hideyuki Matsumura, Commutative ring theory, second ed., Cambridge Studies in Advanced Mathematics, vol. 8, Cambridge University Press, Cambridge, 1989.

[Mur07] Daniel Murfet, Triangulated Categories Part i, Note (2007), 1-88, therisingsea.org/notes/TriangulatedCategories.pdf.

[Nee92] Amnon Neeman, The connection between the K-theory localization theorem of Thomason, Trobaugh and Yao and the smashing subcategories of Bousfield and Ravenel, Ann. Sci. École Norm. Sup. (4) 25 (1992), no. 5, 547-566.

[Nee01] Triangulated categories, Annals of Mathematics Studies, vol. 148, Princeton University Press, Princeton, NJ, 2001.

[Orl92] D. O. Orlov, Projective bundles, monoidal transformations, and derived categories of coherent sheaves, Izv. Ross. Akad. Nauk Ser. Mat. 56 (1992), no. 4, 852-862.

[Orl04] Triangulated categories of singularities and D-branes in Landau-Ginzburg models, Tr. Mat. Inst. Steklova 246 (2004), no. Algebr. Geom. Metody, Svyazi i Prilozh., 240-262.

[Orl12] Dmitri Orlov, Matrix factorizations for nonaffine LG-models, Math. Ann. 353 (2012), no. 1, 95-108.

[OSS11] Christian Okonek, Michael Schneider, and Heinz Spindler, Vector bundles on complex projective spaces, Modern Birkhäuser Classics, Birkhäuser/Springer Basel AG, Basel, 2011.

[Pos11a] Leonid Positselski, Coherent analogues of matrix factorizations and relative singularity categories, arXiv:1102.0261 (2011).

[Pos11b] Leonid Positselski, Two kinds of derived categories, Koszul duality, and comodule-contramodule correspondence, Mem. Amer. Math. Soc. 212 (2011), no. 996, vi+133.

[Sch] Olaf M. Schnürer, in preparation.

[SdSSdS09] Fernando Sancho de Salas and Pedro Sancho de Salas, A direct proof of the theorem on formal functions, Proc. Amer. Math. Soc. 137 (2009), no. 12, 4083-4088.

Department of Mathematics, Indiana University, Rawles Hall, 831 East 3rd Street, BloomINGTON, IN 47405, USA

E-mail address: vlunts@indiana.edu

E-mail address: oschnure@indiana.edu, olaf.schnuerer@math.uni-bonn.de 